\title{
Type la Supernova Rate Measurements to Redshift 2.5 From Candels: Searching for Prompt Explosions in the Early Universe
}

\section{Citation}

Rodney, Steven A., Adam G. Riess, Louis-Gregory Strolger, Tomas Dahlen, Or Graur, Stefano Casertano, Mark E. Dickinson, et al. 2014. "TYPE la SUPERNOVA RATE MEASUREMENTS TO REDSHIFT 2.5 FROM CANDELS: SEARCHING FOR PROMPT EXPLOSIONS IN THE EARLY UNIVERSE." The Astronomical Journal 148 (1): 13. https://doi.org/10.1088/0004-6256/148/1/13.

\section{Permanent link}

http://nrs.harvard.edu/urn-3:HUL.InstRepos:41412124

\section{Terms of Use}

This article was downloaded from Harvard University's DASH repository, and is made available under the terms and conditions applicable to Other Posted Material, as set forth at http:// nrs.harvard.edu/urn-3:HUL.InstRepos:dash.current.terms-of-use\#LAA

\section{Share Your Story}

The Harvard community has made this article openly available.

Please share how this access benefits you. Submit a story.

\section{Accessibility}




\title{
TYPE Ia SUPERNOVA RATE MEASUREMENTS TO REDSHIFT 2.5 FROM CANDELS: SEARCHING FOR PROMPT EXPLOSIONS IN THE EARLY UNIVERSE
}

\author{
Steven A. Rodney ${ }^{1,24}$, Adam G. Riess ${ }^{1,2}$, Louis-Gregory Strolger ${ }^{2}$, Tomas Dahlen ${ }^{2}$, Or Graur ${ }^{1,3,4}$, \\ Stefano Casertano $^{2}$, Mark E. Dickinson ${ }^{5}$, Henry C. Ferguson $^{2}$, Peter Garnavich $^{6}$, Brian Hayden ${ }^{7}$, Saurabh W. Jha $^{8}$, \\ David O. Jones ${ }^{1}$, Robert P. Kirshner ${ }^{9}$, Anton M. Koekemoer ${ }^{2}$, Curtis McCully ${ }^{8}$, Bahram Mobasher ${ }^{10}$, \\ Brandon Patel ${ }^{8}$, Benjamin J. Weiner ${ }^{11}$, S. Bradley Cenko ${ }^{12,13}$, Kelsey I. ClubB ${ }^{14}$, Michael Cooper ${ }^{15}$, \\ Alexei V. Filippenko ${ }^{14}$, Teddy F. Frederiksen ${ }^{16}$, Jens Huorth ${ }^{16}$, Bruno Leibundgut ${ }^{17}, 18$, Thomas Matheson ${ }^{5}$, \\ Hooshang Nayyeri ${ }^{10}$, Kyle Penner ${ }^{11}$, Jonathan Trump ${ }^{19,24}$, Jeffrey M. Silverman ${ }^{20}$, Vivian ${ }^{10}$, K. Azalee Bostroem ${ }^{2}$, \\ Peter Challis $^{9}$, Abhijith Rajan ${ }^{21}$, Schuyler WolfF ${ }^{1,25}$, S. M. Faber ${ }^{22}$, Norman A. Grogin ${ }^{2}$, and Dale Kocevski ${ }^{23}$ \\ ${ }^{1}$ Department of Physics and Astronomy, The Johns Hopkins University, Baltimore, MD 21218, USA \\ ${ }^{2}$ Space Telescope Science Institute, Baltimore, MD 21218, USA \\ ${ }^{3}$ Department of Astrophysics, Tel Aviv University, 69978 Tel Aviv, Israel \\ ${ }^{4}$ Department of Astrophysics, American Museum of Natural History, New York, NY 10024, USA \\ ${ }^{5}$ National Optical Astronomy Observatory, 950 North Cherry Avenue, Tucson, AZ 85719, USA \\ ${ }^{6}$ Department of Physics, University of Notre Dame, Notre Dame, IN 46556, USA \\ ${ }^{7}$ E.O. Lawrence Berkeley National Lab, 1 Cyclotron Road, Berkeley, CA 94720, USA \\ ${ }^{8}$ Department of Physics and Astronomy, Rutgers, The State University of New Jersey, Piscataway, NJ 08854, USA \\ ${ }^{9}$ Harvard-Smithsonian Center for Astrophysics, Cambridge, MA 02138, USA \\ ${ }^{10}$ Department of Physics and Astronomy, University of California, Riverside, CA 92521, USA \\ ${ }^{11}$ Department of Astronomy, University of Arizona, Tucson, AZ 85721, USA \\ ${ }^{12}$ Astrophysics Science Division, NASA Goddard Space Flight Center, Mail Code 661, Greenbelt, MD 20771, USA \\ 13 Joint Space Science Institute, University of Maryland, College Park, MD 20742, USA \\ ${ }^{14}$ Department of Astronomy, University of California, Berkeley, CA 94720, USA \\ ${ }^{15}$ Department Physics and Astronomy, University of California, Irvine, CA 92697, USA \\ ${ }^{16}$ Dark Cosmology Centre, Niels Bohr Institute, University of Copenhagen, Juliane Maries Vej 30, DK-2100 Copenhagen, Denmark \\ ${ }^{17}$ European Southern Observatory, Garching bei München, Germany \\ ${ }^{18}$ Excellence Cluster Universe, Technische Universität München, Germany \\ ${ }^{19}$ Department of Astronomy and Astrophysics, Pennsylvania State University, University Park, PA 16802, USA \\ ${ }^{20}$ Department of Astronomy, University of Texas, Austin, TX 78712, USA \\ ${ }^{21}$ School of Earth and Space Exploration, Arizona State University, Tempe, AZ 85287, USA \\ 22 Department of Astronomy and Astrophysics, University of California, Santa Cruz, CA 92064, USA \\ ${ }^{23}$ Department of Physics and Astronomy, University of Kentucky, Lexington, KY 40506, USA \\ Received 2013 November 10; accepted 2014 February 26; published 2014 June 4
}

\begin{abstract}
The Cosmic Assembly Near-infrared Deep Extragalactic Legacy Survey (CANDELS) was a multi-cycle treasury program on the Hubble Space Telescope (HST) that surveyed a total area of $\sim 0.25 \mathrm{deg}^{2}$ with $\sim 900 H S T$ orbits spread across five fields over three years. Within these survey images we discovered 65 supernovae (SNe) of all types, out to $z \sim 2.5$. We classify $\sim 24$ of these as Type Ia SNe (SNe Ia) based on host galaxy redshifts and SN photometry (supplemented by grism spectroscopy of six SNe). Here we present a measurement of the volumetric SN Ia rate as a function of redshift, reaching for the first time beyond $z=2$ and putting new constraints on SN Ia progenitor models. Our highest redshift bin includes detections of SNe that exploded when the universe was only $\sim 3$ Gyr old and near the peak of the cosmic star formation history. This gives the CANDELS high redshift sample unique leverage for evaluating the fraction of SNe Ia that explode promptly after formation $(<500 \mathrm{Myr})$. Combining the CANDELS rates with all available SN Ia rate measurements in the literature we find that this prompt SN Ia fraction is $f_{P}=0.53_{\text {stat } 0.10 \text { sys } 0.26}^{ \pm 0.09}$, consistent with a delay time distribution that follows a simple $t^{-1}$ power law for all times $t>40$ Myr. However, mild tension is apparent between ground-based low- $z$ surveys and space-based high- $z$ surveys. In both CANDELS and the sister HST program CLASH (Cluster Lensing And Supernova Survey with $H u b b l e$ ), we find a low rate of SNe Ia at $z>1$. This could be a hint that prompt progenitors are in fact relatively rare, accounting for only $20 \%$ of all SN Ia explosions - though further analysis and larger samples will be needed to examine that suggestion.
\end{abstract}

Key words: infrared: general - supernovae: general - surveys

Online-only material: color figures

\section{INTRODUCTION}

The prevailing model for a Type Ia supernova (SN Ia) progenitor system begins with a binary system in which the primary star evolves to become a white dwarf (WD). The WD

\footnotetext{
${ }^{24}$ Hubble Fellow.

${ }^{25}$ NSF Postdoctoral Fellow.
}

acquires mass from its companion star, approaches the Chandrasekhar limit, and explodes in a thermonuclear runaway (see Hillebrandt \& Niemeyer 2000; Livio 2001, for reviews). The companion star that feeds the WD and thereby sets off the thermonuclear bomb is one of the key components of this model, but remains a topic of ongoing debate. In single degenerate (SD) models, the companion is a main sequence or evolved giant star, transferring mass via Roche lobe overflow, stellar winds, 


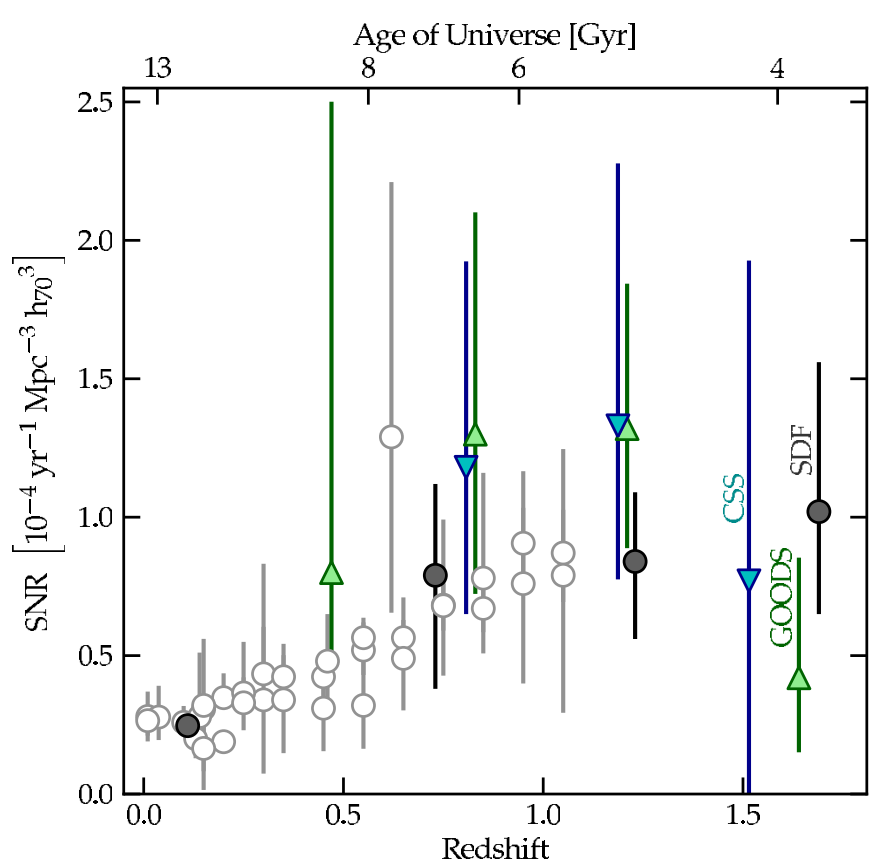

Figure 1. Volumetric SN Ia rates before completion of the CANDELS and CLASH SN surveys. Assorted ground-based surveys are plotted as white circles (Blanc et al. 2004; Botticella et al. 2008; Cappellaro et al. 1999; Dilday et al. 2010; Hardin et al. 2000; Horesh et al. 2008; Graur \& Maoz 2013; Li et al. 2011; Melinder et al. 2012; Pain et al. 2002; Perrett et al. 2012; Rodney \& Tonry 2010; Tonry et al. 2003). Three high redshift SN surveys are highlighted: gray circles for the Subaru Deep Field (SDF; Graur et al. 2011), blue downward triangles for volumetric (not cluster) rates from the Cluster Supernova Survey (CSS; Barbary et al. 2012), and green upward triangles for the GOODS and PANS surveys (Dahlen et al. 2008).

(A color version of this figure is available in the online journal.)

or other means (Whelan \& Iben 1973). In double degenerate (DD) models the companion is another WD, merging with the primary after a period of orbital decay driven by gravitational wave radiation (Iben \& Tutukov 1984; Webbink 1984). More recent variations on these pathways to explosion include the "core degenerate" scenario (Kashi \& Soker 2011) and perturbationinduced mergers in triple systems (Thompson 2011).

The SN Ia explosion rate as a function of redshift, $\operatorname{SNR}(z)$, can provide an important observational test to constrain SN Ia progenitor models and possibly distinguish between them. In this paper we will present measurements of the SN Ia rate as a function of redshift and use them to place new constraints on $\mathrm{SN}$ Ia progenitor models, particularly on the fraction of SN Ia progenitors that explode within $500 \mathrm{Myr}$ after their formation.

Suppose we have a burst of star formation in a galaxy, such that the star formation rate can be approximated by a delta function in time. Binary population synthesis modeling gives us the initial conditions of all the binaries (mass, orbital separation, etc.), and a progenitor model sets the conditions necessary for explosion as an SN Ia. Using a stellar evolution model, one can follow the binary systems as they evolve, measuring the delay time distribution (DTD) between formation and explosion. To put constraints on SN Ia progenitor models, we can translate this DTD to cosmic scales and compare it to the observed volumetric SN Ia rate as a function of look-back time, as first proposed by Madau et al. (1998).

As shown in Figure 1, recent measurements of the $\mathrm{SN}$ Ia rate at low redshift $(z<1)$ are in good agreement, consistently finding that the $\operatorname{SNR}(z)$ rises steadily to at least $z \sim 1$ (e.g., Rodney \&
Tonry 2010; Dilday et al. 2010; Perrett et al. 2012). However, at $z>1$ the trend of the $\operatorname{SNR}(z)$ curve is much less clear. The spectral energy distribution of an SN Ia peaks in the rest-frame $B$ band with an absolute magnitude around -19.5 . At $z=1.2$ that peak brightness becomes fainter than 25 th magnitude in the observer's $z$ band - making discovery and light curve follow-up nearly impossible for ground-based observatories.

For that reason, space-based surveys using the Hubble Space Telescope's Advanced Camera for Surveys (HST+ACS) have been the primary vehicle for tracking the $\operatorname{SNR}(z)$ to $z \sim 1.5$. The GOODS+PANS surveys were the first programs to extend rate measurements beyond $z \sim 1$ (Dahlen et al. 2004; Dahlen et al. 2008), and their measured rates suggested a peak in the SN Ia rate at $z \sim 1.2$, with a decline at higher redshifts. Independent examination of the same survey data recovered the same trend (Kuznetsova et al. 2008), although both analyses were limited by a small sample size in the highest redshift bin. Subsequently, the Cluster Supernova Survey (CSS) of the Supernova Cosmology Project used HST+ACS to measure the volumetric SN Ia rate (Barbary et al. 2012). These data revealed a similar peak and decline, although with even larger uncertainty in the high- $z$ bins. From the ground, the Subaru Deep Field (SDF) SN survey used the Suprime-cam imager on the Subaru telescope to reach similar redshifts (Poznanski et al. 2007; Graur et al. 2011). As can be seen in Figure 1, these SDF rates formally show no decline in the highest redshift bin, but they are consistent with the HST+ACS results, within the errors.

The HST-ACS high- $z$ SNe Ia generally have reliable classifications, based on well-sampled multi-band light curves, spectroscopic redshifts, and HST grism spectroscopy of most SN Ia candidates. However, due to the relatively small survey area, these programs have very large statistical uncertainties (Dahlen et al. (2008) have 3 SNe in their highest redshift bin; Barbary et al. (2012) have $\sim 1$ ). In contrast, the SDF survey built up a larger sample (10 SNe Ia at $z \sim 1.5$ ) but their survey design introduced potential for large systematic biases. The SDF epochs were spaced by $\sim 1$ year, meaning that the phase of the SN light curve at discovery was unconstrained, and the classification of detected $\mathrm{SNe}$ was based on only a single epoch of photometric data in the $R, i^{\prime}, z^{\prime}$ bands. Furthermore, redshifts for the SDF high- $z$ SN sample were based almost exclusively on photometric redshift estimates of the SN host galaxies, not as precise or reliable as spectroscopic redshifts-but see Frederiksen et al. (2014) for one spectroscopic confirmation of an SDF host galaxy at $z=1.55$.

An apparent peak in the $\mathrm{SN}$ Ia rate at $z \sim 1$ and a decline toward $z=1.5$ has been interpreted as indicating a delay of $>1$ Gyr between formation and explosion for most SN Ia (Strolger et al. 2004; Strolger et al. 2010). This would be broadly consistent with some SD models, and inconsistent with DD models, which typically predict a large fraction of SNe Ia that explode promptly after star formation (within $1 \mathrm{Gyr}$ ). A clear measurement of the shape of the SN Ia rate function at $z>1$ would provide an important constraint on DTD models, and would go a long way toward resolving the question of whether an SD or DD model could be the dominant progenitor channel for all SNe Ia at all redshifts. Given the problems with current high- $z$ SN rates, there is a clear need to improve the measurement by expanding the sample of well-classified SNe at $z>1$.

In this paper we present a measurement of the $\operatorname{SNR}(z)$ from a sample of $65 \mathrm{SNe}$ discovered in the CANDELS SN program, extending the $\operatorname{SNR}(z)$ measurement for the first time to $z=2.5$. 
Table 1

CANDELS SN Survey Fields ${ }^{\mathrm{a}}$

\begin{tabular}{|c|c|c|c|c|c|}
\hline Field & $\begin{array}{l}\text { R.A. } \\
(\mathrm{J} 2000)\end{array}$ & $\begin{array}{c}\text { Decl. } \\
(\mathrm{J} 2000)\end{array}$ & $\begin{array}{l}\text { WFC3-IR } \\
\text { Tiles/Epoch }\end{array}$ & $\begin{array}{l}\text { Searchable Area } \\
\quad\left(\operatorname{arcmin}^{2}\right)\end{array}$ & 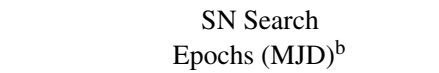 \\
\hline COSMOS & $10: 00: 28$ & $+02: 12: 04$ & 44 & $196.8\left(9^{\prime} \times 22^{\prime}\right)$ & [55905], 55953 \\
\hline EGS-A & $14: 19: 18$ & $+52: 49: 30$ & 25 & $106.2\left(\frac{1}{2} \text { of } 7^{\prime} \times 32^{\prime}\right)^{\mathrm{c}}$ & {$[55653], 55703$} \\
\hline EGS-B & $14: 19: 18$ & $+52: 49: 30$ & 20 & $92.9\left(\frac{1}{2} \text { of } 7^{\prime} \times 32^{\prime}\right)^{\mathrm{c}}$ & {$[56387], 56437$} \\
\hline UDS & $02: 17: 38$ & $-05: 12: 00$ & 44 & $207.1\left(9^{\prime} \times 22^{\prime}\right)$ & {$[55512], 55562$} \\
\hline GOODS-S Wide & $03: 32: 42$ & $-27: 53: 37$ & $\sim 8$ & $39.4\left(4^{\prime} \times 10^{\prime}\right)$ & {$[55573], 55621$} \\
\hline GOODS-N Wide NE & $12: 37: 29$ & $+62: 18: 40$ & $\sim 8$ & $38.1\left(4^{\prime} \times 10^{\prime}\right)$ & {$[56183], 56238$} \\
\hline GOODS-N Wide SW & $12: 36: 20$ & $+62: 10: 25$ & $\sim 10$ & $49.5\left(5^{\prime} \times 10^{\prime}\right)$ & {$[56020], 56073$} \\
\hline GOODS-N Deep & $12: 36: 55$ & $+62: 14: 19$ & $\sim 15$ & $66.8\left(7^{\prime} \times 10^{\prime}\right)^{\mathrm{d}}$ & $\begin{array}{l}{[56020], 56073,56126,56183,56238,} \\
56297,56348,56402,56458,56511\end{array}$ \\
\hline
\end{tabular}

Notes.

${ }^{a}$ Coordinates give approximate center of each CANDELS IR survey field.

${ }^{\mathrm{b}}$ Mean date of observation epoch. First epoch listed [in brackets] provided IR template images.

c The CANDELS EGS field was divided into two interlocking halves, observed separately in 2011 and 2013. See Grogin et al. (2011) for details.

$\mathrm{d}$ The deep field search areas vary by epoch. The given value reflects the average.

This SN survey is a joint operation of two HST Multi-Cycle Treasury (MCT) programs: the Cosmic Assembly Near-infrared Deep Extragalactic Legacy Survey (CANDELS; PIs: Faber and Ferguson; Grogin et al. 2011; Koekemoer et al. 2011), and the Cluster Lensing and Supernovae search with Hubble (CLASH; PI: Postman; Postman et al. 2012). The SN discovery and followup for both programs were allocated to the HST MCT SN program (PI: Riess). The results presented here are based on the full five fields and $\sim 0.25 \mathrm{deg}^{2}$ of the CANDELS program, observed from 2010 to 2013. A companion paper presents the SN Ia rates from the CLASH sample (Graur et al. 2014). A composite analysis that combines the CANDELS+CLASH SN sample and revisits past $H S T$ surveys will be presented in a future paper.

In Section 2 we describe the SN search component of the CANDELS survey, and in Section 3 we describe our detection efficiency measurements. Our photometric SN classifications are presented in Section 4, properties of the $\mathrm{SN}$ host galaxies are described in Section 5, and in Section 6 we detail new grism spectroscopy for four of our SNe. The rate calculation is described in Section 7 and we discuss the consequences for SN Ia progenitor models in Section 8. Finally, a summary is presented in Section 9. In tables and figures throughout the paper, we present the subset of $14 \mathrm{SNe}$ with $z>1.5$ in the main body of the text, with the remaining 51 shown in Appendix B. Throughout this work we assume a flat $\Lambda \mathrm{CDM}$ cosmology with $H_{0}=70, \Omega_{\mathrm{m}}=0.3$, and $\Omega_{\Lambda}=0.7$.

\section{THE CANDELS SN SURVEY}

The three-year CANDELS program was designed to probe galaxy evolution out to $z \approx 8$ with deep infrared (IR) and optical imaging of five well-studied extragalactic fields: GOODS-S, GOODS-N, COSMOS, UDS, and EGS. ${ }^{26}$ As described fully in Grogin et al. (2011), the CANDELS program includes both "wide" and "deep" fields. The wide component of CANDELS comprises the COSMOS, UDS, and EGS fields, plus one-third of the GOODS-S field and one half of the GOODS-N field - a total

\footnotetext{
26 GOODS-S/N: the Great Observatories Origins Deep Survey South and North (Giavalisco et al. 2004); COSMOS: the Cosmic Evolution Survey (Scoville et al. 2007; Koekemoer et al. 2007); UDS: the UKIDSS Ultra Deep Survey (Lawrence et al. 2007; Cirasuolo et al. 2007); EGS: the Extended Groth Strip (Davis et al. 2007).
}

Table 2

Typical Exposures for a Single SN Search Epoch

\begin{tabular}{lccc}
\hline \hline Camera & Filter & $\begin{array}{c}\text { Exposures } \\
\left(N_{\exp } \times \mathrm{s}\right)\end{array}$ & $\begin{array}{c}\text { Limiting } \\
\text { Magnitude }^{\mathrm{a}}\end{array}$ \\
\hline WFC3-IR & F160W $(H)$ & $2 \times 600$ & 25.4 \\
WFC3-IR & F125W $(J)$ & $2 \times 500$ & 25.8 \\
WFC3-UVIS & F350LP $(W)$ & $1 \times 430$ & 27.8 \\
ACS-WFC & F814W $(I)$ & $2 \times 700$ & 27.3 \\
ACS-WFC & F606W $(V)$ & $2 \times 350$ & 28.1 \\
\hline
\end{tabular}

Note. ${ }^{\text {a }}$ Vega magnitude that yields $\mathrm{S} / \mathrm{N} \sim 5$ in the given exposure sequence.

survey area of $730 \mathrm{arcmin}^{2}$. The CANDELS survey provides two visits to each wide field, spaced by $\sim 50$ days. The "deep" component of CANDELS came from the central $67 \mathrm{arcmin}^{2}$ of each of the GOODS-S and GOODS-N fields. These deep regions were each visited 15 times over the course of two years (2010-2012 for GOODS-S, 2012-2013 for GOODS-N). Only 10 of those visits are used for SN discovery (the other visits lack template data for generating difference images), and those 10 epochs are also spaced at a cadence of $\sim 50$ days. The CANDELS fields analyzed in this work are described in Table 1.

Table 2 presents the exposure times and $5 \sigma$ limiting magnitudes for a typical single-epoch set of exposures. Each CANDELS visit includes a set of four infrared exposures from the Wide Field Camera 3 (WFC3) IR detector: two in F160W ( $H$ band) and two in F125W ( $J$ band). These are the search filters for the CANDELS SN survey (i.e., all SNe in our sample are infrared detections). Additionally, each observation set includes a broad optical band, which helps to distinguish SNe Ia from Core Collapse Supernovae (CCSNe) and other transients (see Section 4). In $\sim 80 \%$ of the SN search visits, this blue component is collected within minutes of the IR exposures as a single exposure using the WFC3 UVIS camera in the F350LP filter (a broad "white light" filter that we refer to as the " $W$ band"). In the remaining $\sim 20 \%$ of visits (in the wide fields) the $W$ band exposure is replaced with ACS observations in the F606W filter (broad $V$ band), and complemented by the ACS F814W filter (broad $I$ band). These ACS observations come from coordinated parallel visits and are taken within three days of the primary IR visit. 
In addition to the $\sim 750 \mathrm{HST}$ orbits devoted to survey imaging in the CANDELS program, an additional 150 HST orbits were allocated for target of opportunity (ToO) follow-up observations of newly discovered SNe. Another 52 orbits were provided by the CLASH program, so the total CANDELS+CLASH SN follow-up allocation was 202 orbits. These follow-up visits provided supplementary imaging and slitless spectroscopy observations to aid in the classification of SN candidates, and to measure the light curves of SNe Ia, allowing distance determinations for cosmology.

\subsection{Data Processing Pipeline}

All CANDELS survey images were processed through a data processing pipeline optimized for the detection of SNe by human searchers. This pipeline is similar in function to the CANDELS and CLASH pipelines (Koekemoer et al. 2011; Postman et al. 2012), but includes some important differences specific to the $\mathrm{SN}$ search. There are four principal components in the pipeline: calibration, image combination, template subtraction, and fake SN planting.

In the calibration stage, RAW images from $H S T$ are processed into FLT images using the STSDAS calibration tools provided by the Space Telescope Science Institute. ${ }^{27}$ This includes bias correction, dark subtraction, flat fielding, and "up-the-ramp" fitting for cosmic ray rejection, as appropriate for each camera and detector.

The image combination step uses the MultiDrizzle software (Koekemoer et al. 2002; Fruchter \& Hook 2002) to combine multiple dithered images in the same filter from the same observing epoch, while also removing the geometric distortion of the HST focal plane. For each drizzled WFC3-IR image, we then generate a template image that combines all intersecting images from the prior epoch(s). These components of the template image are astrometrically registered using catalog matching to align them with the WFC3-IR image of the current epoch. The astrometric registration for the SN search is done tile-by-tile and the output pixel grid is left in the natural unrotated frame of the observation. This contrasts with the CANDELS mosaic imaging pipeline (Koekemoer et al. 2011), which constructs a global astrometric solution across the whole field, and rotates every image to put north up and east to the left. These choices for the SN pipeline are designed to maximize the precision of the local inter-epoch registrations and to minimize dilution of the already undersampled point-spread function (PSF) for single-visit drizzled images.

Next, each template image is subtracted from the corresponding search epoch image, producing the difference images for SN discovery. Due to the very stable PSF of HST, the CANDELS images do not require any convolution with a PSF kernel to match conditions across epochs (Alard \& Lupton 1998), as is commonly done in ground-based SN surveys. The CANDELS visits were constructed with small positioning shifts after each exposure, such that the two $H$ band and two $J$ band exposures together formed a four-point "box" dither pattern. This yields better sampling of the PSF and helps in the removal of detector artifacts from the final combined image. To take advantage of the full dither sequence, our SN searching was primarily done on a combined " $J+H$ " image-simply the sum of the F125W and F160W difference images for each epoch.

In the final stage of the data processing pipeline, we reprocess all the search epoch data, this time with fake SNe planted into

27 http://www.stsci.edu/institute/software_hardware/pyraf/stsdas the WFC3-IR survey images. These synthetic SNe enable a direct measurement of the detection efficiency of our human searchers (see Section 3). Each fake SN consists of a small image $(\sim 50 \times 50$ pixels $)$ of a simulated point source, generated using the TinyTim software (Krist et al. 2011). The fake SN images are added to the WFC3-IR images at the FLT stage, after image calibration and before drizzling. These "faked" FLT files are then redrizzled, and the existing template images are subtracted off, resulting in a parallel set of "faked" difference images.

\subsection{SN Discovery}

To find SN candidates in the CANDELS WFC3-IR difference images, we used human searchers, who scanned each image by eye to detect significant deviations from the noise. We had $\sim 20$ individuals regularly engaged in searching the CANDELS data, and searching tasks were assigned so that every WFC3-IR tile was examined by at least two people. Searchers recorded the position of all potential transient object detections, and assigned a quality grade. All transient sources that received a high- or moderate-quality grade were carefully vetted to pare down the list to transient sources that are very likely real SNe. The criteria for inclusion in this SN candidate list are: (1) a profile consistent with a point source, (2) detected in the $J+H$ difference image and also individually in both the $J$ and $H$ bands, and (3) clean of evidence indicating that it could be a detector artifact (neighboring bad pixels, on the detector edge, etc.). For the rate analysis presented here, we also require that the object reached its peak magnitude in IR bands after the $H S T$ template images were collected (i.e., we reject any SN that has less flux in the search epoch than in the template epoch).

Finally, we also discard a total of six objects that are positively classified as AGNs. These six were located at the center of a host galaxy that has observational indicators to classify it as an AGN (X-ray emission, spectral line broadening, prior optical/IR variability, etc.). Our final sample contains $65 \mathrm{SN}$ candidates that meet these requirements. Table 3 lists the $14 \mathrm{SNe}$ at redshifts $z>1.5$, and Table 9 lists the remaining 51 SNe. In keeping with the practice of past HST SN surveys, we assign each SN a unique eight-digit name that indicates the field and the year of discovery, with the final three letters referencing our team's internal "nickname" for each object. ${ }^{28}$ Figure 2 shows "postage stamp images" with the detection images for the $14 \mathrm{SNe}$ at $z>1.5$, and the remainder are in the Appendix, in Figures 13 and 14 .

\subsection{Follow-up Observations}

Upon discovery, every SN was evaluated for possible followup observations with HST or ground-based telescopes. First, a redshift probability density function (PDF) was assigned, using pre-existing spectroscopy of the host galaxy when available and a photometric redshift (photo- $z$ ) when not. The photo- $z$ estimates were derived from template fitting to the observed spectral energy distribution (SED) of each SN host galaxy (Dahlen et al. 2013). Then a preliminary SN classification (Ia or CC) was assigned by comparing the color and magnitude of the observed SN against a sample of synthetic SNe with redshifts drawn from the best available redshift PDF. These synthetic SNe were generated with the SuperNova ANAlysis software (SNANA; Kessler et al. 2009b) (see Section 4 for more details).

28 The nicknames for the CANDELS SNe are mostly derived from U.S.
Presidents and other prominent figures from U.S. history. 


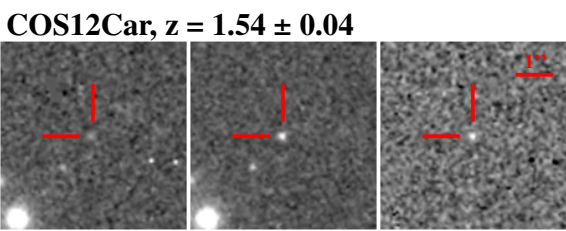

GND13War, $\mathrm{z}=1.689$

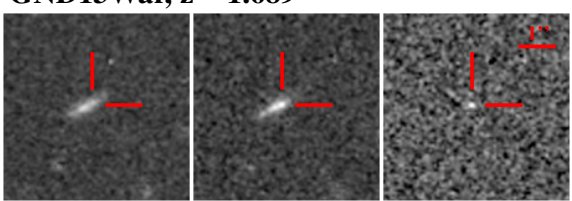

GND13Sto, $\mathrm{z}=1.83 \pm 0.10$

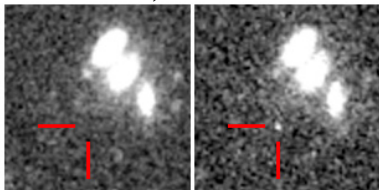

GND12Fai, $z=1.92 \pm 0.07$
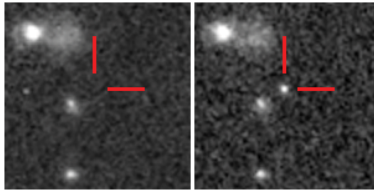

GSD12Hum, $z=2.343$

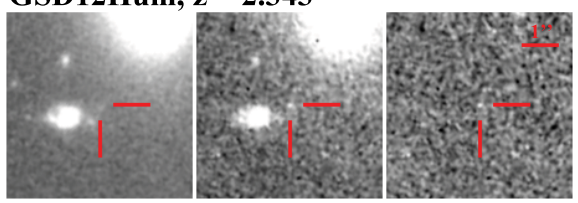

GSD10Pri, $z=1.5499$

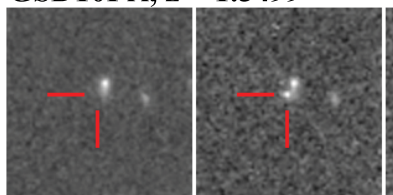

GSD11Bus, $\mathrm{z}=1.7 \pm 0.1$

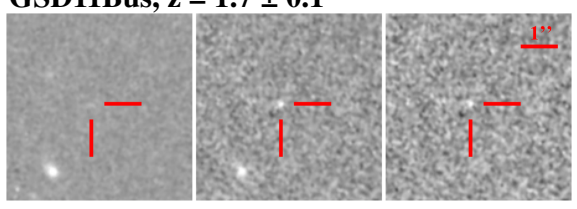

GND12Bre, $z=1.88$

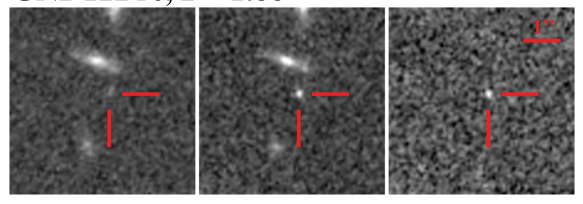

GND12Col, $\mathrm{z}=2.24 \pm 0.04$

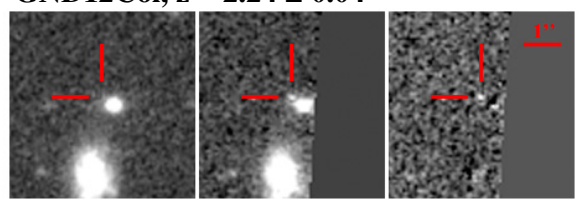

GSD12Qua, $z=2.37$

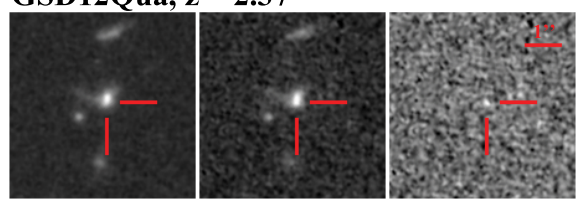

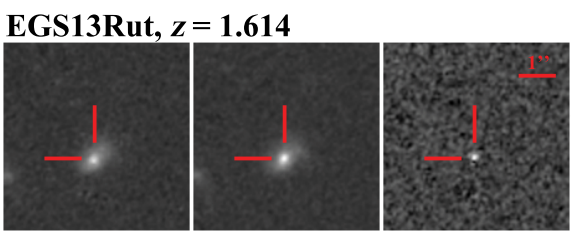

UDS11Hug, $\mathrm{z}=1.76 \pm 0.03$

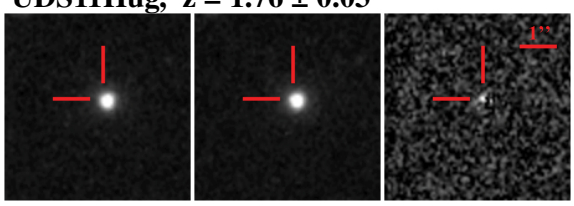

UDS10Wil, $z=1.914$

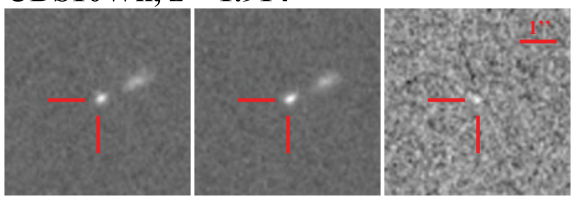

EGS11Tyl, $z=2.24 \pm 0.10$

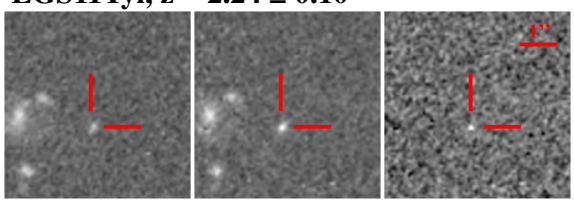

Figure 2. Detection images for $14 \mathrm{SN}$ from the CANDELS fields with redshifts $z>1.5$. Each image triplet shows $H$ band (F160W) images with the template image on the left, the discovery epoch image in the middle, and the difference image on the right. All images have a width of about 6 arcsec, with north up and east to the left. The position of the SN is marked by (red) crosshairs in every frame. Discovery images for the other $51 \mathrm{SN}$ with $z<1.5$ are provided in Appendix B.

(A color version of this figure is available in the online journal.)

Any SNe with a redshift $z>1$ and a color consistent with an SN Ia classification were then considered for possible followup with $H S T$. Where necessary and whenever possible, the host galaxies of these high-priority targets were quickly observed (within $\sim 1$ week of discovery) with ToO spectroscopic observations using ground-based observatories (primarily Gemini, Keck, and the Very Large Telescope (VLT)). The host galaxies of other SN candidates (CCSN and those with $z<1$ ) were targeted for later spectroscopic observations from the ground to determine precise redshifts, all reported in Table 4 (and in Table 10).

Some of the most promising candidates for classification as SNe Ia at $z>1.5$ were selected for supplementary imaging and/or grism spectroscopy with HST. Two of these, SNe GSD10Pri and UDS10Wil, have been presented elsewhere (Rodney et al. 2012; Jones et al. 2013). Due to the high cost of grism observations (at least $10 H S T$ orbits are required to reach sufficient $\mathrm{S} / \mathrm{N}$ in distant $\mathrm{SNe}$ ), we applied strict criteria for selecting grism targets: (1) best available redshift $z>1$, preferably $z>1.5$; (2) observed SN colors consistent with a (possibly reddened) SN Ia at that redshift; (3) observed SN magnitudes within $\sim 1.5 \mathrm{mag}$ of an SN Ia at that redshift (i.e., using a very weak prior around a standard $\Lambda \mathrm{CDM}$ cosmology); and (4) SN position allows for a grism observation without severe contamination.

Without a slit to isolate the SN light in WFC3-IR grism spectroscopy, a high- $z$ SN Ia candidate can most productively be observed if the trace of the SN spectrum can be positioned to avoid contamination from nearby galaxies. Thus, to satisfy the final criterion (4), the candidate must be well separated from the core of its host, or located in a host that is faint relative to the $\mathrm{SN}$. We also require an orientation angle that avoids contamination of the SN spectral trace from the zeroth order and first order light of other nearby stars and galaxies. Of course, this orientation must also be accessible to HST at the time of observation, with suitable guide stars in range. In practice, these criteria were satisfied for only six SN candidates. The results of those observations are described in Section 6 . Another 37 CANDELS SNe were followed with ToO imaging observations. These imaging targets included SN Ia candidates that satisfied some or all of the first three criteria, but were not suitable for grism observations, as well as some likely CCSNe that we were able to include in the same field of view as those primary targets.

\section{DETECTION EFFICIENCY}

Translating SN detections into an SN rate measurement requires characterization of the survey detection efficiency, i.e., the fraction of SNe that are detected by our human searchers. This recovery fraction is most strongly influenced by the $\mathrm{S} / \mathrm{N}$ of the object in the WFC3-IR difference images. The SN host galaxy is also an important factor affecting SN detectability, as we discuss further in Section 3.1. 
Table 3

14 Supernovae with $z>1.5$ (See Appendix B for the Remainder)

\begin{tabular}{|c|c|c|c|c|c|c|c|}
\hline Name & $\begin{array}{c}\text { R.A. } \\
\text { (J2000) }\end{array}$ & $\begin{array}{c}\text { Decl. } \\
(\mathrm{J} 2000)\end{array}$ & $P\left(\mathrm{Ia} \mid D_{z}\right)^{\mathrm{a}}$ & $P\left(\mathrm{Ia} \mid D_{\text {host }}\right)^{\mathrm{b}}$ & $z_{\mathrm{SN}}{ }^{\mathrm{c}}$ & $( \pm)$ & $z$ Source $^{\mathrm{d}}$ \\
\hline COS12Car & $10: 00: 14.726$ & $+02: 11: 32.57$ & $0.62_{-0.36}^{+0.09}$ & $0.80_{-0.07}^{+0.00}$ & 1.54 & $(0.04)$ & $\overline{\mathrm{SN} \text { spec}}-z+\mathrm{SN}$ phot $-z$ \\
\hline GSD10Pri & $03: 32: 38.010$ & $-27: 46: 39.08$ & $1.00_{-0.00}^{+0.00}$ & $1.00_{-0.00}^{+0.00}$ & 1.545 & $(0.001)$ & host+SN spec- $z$ \\
\hline EGS13Rut & $14: 20: 48.106$ & $+53: 04: 22.12$ & $1.00_{-0.00}^{+0.00}$ & $1.00_{-0.00}^{+0.00}$ & 1.614 & $(0.005)$ & host spec- $z+\mathrm{SN}$ phot $-z$ \\
\hline GND13War & $12: 36: 54.761$ & $+62: 12: 16.70$ & $0.01_{-0.01}^{+0.02}$ & $0.01_{-0.00}^{+0.00}$ & 1.689 & $(0.005)$ & host spec- $z$ \\
\hline GSD11Bus & $03: 32: 42.776$ & $-27: 48: 07.10$ & $0.00_{-0.00}^{+0.00}$ & $0.00_{-0.00}^{+0.00}$ & 1.7 & $(0.1)$ & host+SN phot- $z$ \\
\hline UDS11Hug & $02: 17: 37.427$ & $-05: 08: 41.43$ & $0.82_{-0.21}^{+0.05}$ & $1.00_{-0.00}^{+0.00}$ & 1.761 & $(0.025)$ & host+SN phot- $z$ \\
\hline GND13Sto & $12: 37: 16.778$ & $+62: 16: 41.43$ & $1.00_{-0.00}^{+0.00}$ & $1.00_{-0.00}^{+0.00}$ & 1.83 & $(0.10)$ & host+SN phot- $z$ \\
\hline GND12Bre & $12: 36: 55.520$ & $+62: 13: 58.82$ & $0.00_{-0.00}^{+0.00}$ & $0.00_{-0.00}^{+0.00}$ & 1.880 & $(0.001)$ & host spec- $z$ \\
\hline UDS10Wil & $02: 17: 46.336$ & $-05: 15: 24.00$ & $1.00_{-0.00}^{+0.00}$ & $1.00_{-0.00}^{+0.00}$ & 1.914 & $(0.001)$ & host+SN spec- $z$ \\
\hline GND12Fai & $12: 36: 15.822$ & $+62: 15: 56.50$ & $0.00_{-0.00}^{+0.00}$ & $0.00_{-0.00}^{+0.00}$ & 1.92 & $(0.07)$ & host+SN phot-z \\
\hline GND12Col & $12: 36: 37.569$ & $+62: 18: 32.93$ & $1.00_{-0.01}^{+0.00}$ & $1.00_{-0.00}^{+0.00}$ & 2.24 & $(0.04)$ & host $+\mathrm{SN}$ phot- $z$ \\
\hline EGS11Tyl & $14: 20: 12.944$ & $+52: 57: 10.60$ & $0.24_{-0.15}^{+0.13}$ & $0.57_{-0.04}^{+0.03}$ & 2.244 & $(0.095)$ & host $+\mathrm{SN}$ phot $-z$ \\
\hline GSD12Hum & $03: 32: 15.500$ & $-27: 50: 50.02$ & $0.00_{-0.00}^{+0.00}$ & $0.00_{-0.00}^{+0.00}$ & 2.343 & $(0.001)$ & host spec- $z$ \\
\hline GSD12Qua & $03: 32: 11.723$ & $-27: 49: 11.72$ & $0.00_{-0.00}^{+0.00}$ & $0.00_{-0.00}^{+0.00}$ & 2.370 & $(0.001)$ & host spec- $z$ \\
\hline
\end{tabular}

Notes.

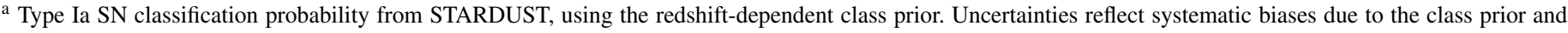
extinction assumptions (Sections 4.2 and 4.3).

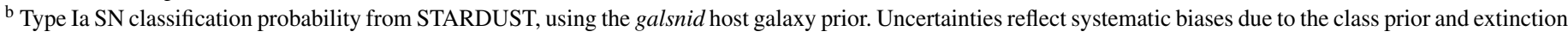
assumptions.

c Posterior redshift and uncertainty, as determined by the STARDUST light curve fit.

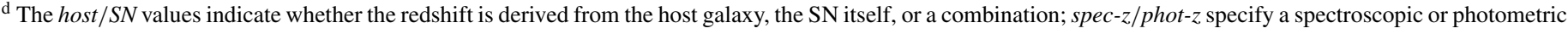
redshift. A value of host $+S N$ phot $z$ means the redshift is derived from a STARDUST light curve fit, with the host galaxy phot- $z$ used as a prior.

Table 4

Host Galaxies of 14 Supernovae with $z>1.5$ (See Appendix B for the Remainder)

\begin{tabular}{|c|c|c|c|c|c|c|c|c|c|}
\hline SN & $\begin{array}{c}\text { R.A. } \\
(\mathrm{J} 2000)\end{array}$ & $\begin{array}{c}\text { Decl. } \\
(\mathrm{J} 2000)\end{array}$ & $\begin{array}{c}d \\
\left({ }^{\prime \prime}\right)\end{array}$ & $\begin{array}{c}d \\
(\mathrm{kpc})^{\mathrm{a}}\end{array}$ & Morph. ${ }^{b}$ & $\mathrm{SED}^{\mathrm{c}}$ & $z_{\text {host }}$ & $( \pm)$ & $z$ Reference $^{\mathrm{d}}$ \\
\hline COS12Car & $\cdots$ & $\cdots$ & $\cdots$ & $\cdots$ & $\cdots$ & $\cdots$ & $\cdots$ & $\cdots$ & $\cdots$ \\
\hline GSD10Pri & $03: 32: 37.991$ & $-27: 46: 38.69$ & 0.46 & 9.6 & $\mathrm{i}$ & SB & 1.545 & 0.001 & Frederiksen et al. (2012) \\
\hline EGS13Rut & $14: 20: 48.113$ & $+53: 04: 22.07$ & 0.08 & 1.7 & d & A & 1.614 & 0.005 & HST+WFC3 (A. Riess) \\
\hline GND13War & $12: 36: 54.787$ & $+62: 12: 16.60$ & 0.21 & 4.3 & di & SB & 1.689 & 0.005 & HST+WFC3 (B. Weiner) \\
\hline GSD11Bus & $03: 32: 42.776$ & $-27: 48: 07.10$ & 0.00 & 0.0 & $\mathrm{u}$ & A & 1.76 & 0.53 & phot- $z$ (T. Dahlen) \\
\hline UDS11Hug & $02: 17: 37.415$ & $-05: 08: 41.53$ & 0.21 & 4.2 & $\mathrm{~s}$ & $\mathrm{P}$ & 1.82 & 0.13 & phot-z (T. Dahlen) \\
\hline GND13Sto & $12: 37: 16.823$ & $+62: 16: 42.65$ & 1.26 & 25.5 & $\mathrm{u}$ & A & 1.8 & 1.2 & phot- $z$ (T. Dahlen) \\
\hline GND12Bre & $12: 36: 55.520$ & $+62: 13: 58.79$ & 0.03 & 0.6 & $\mathrm{i}$ & SB & 1.880 & 0.005 & Keck+MOSFIRE (J. Trump) \\
\hline UDS10Wil & $02: 17: 46.332$ & $-05: 15: 23.90$ & 0.12 & 2.4 & $\mathrm{~s}$ & SB & 1.914 & 0.001 & Jones et al. (2013) \\
\hline GND12Fai & $12: 36: 15.934$ & $+62: 15: 55.91$ & 0.98 & 19.9 & sd & SB & 1.77 & 0.25 & phot $z$ (T. Dahlen) \\
\hline GND12Col & $12: 36: 37.514$ & $+62: 18: 32.66$ & 0.47 & 9.6 & $\mathrm{~s}$ & A & 2.1 & 0.2 & phot- $z$ (T. Dahlen) \\
\hline EGS11Tyl & $14: 20: 12.938$ & $+52: 57: 10.62$ & 0.06 & 1.2 & sd & SB & 1.95 & 0.45 & phot- $z$ (T. Dahlen) \\
\hline GSD12Hum & $03: 32: 15.585$ & $-27: 50: 50.43$ & 1.20 & 24.3 & di & SB & 2.343 & 0.001 & Balestra et al. (2010) \\
\hline GSD12Qua & $03: 32: 11.713$ & $-27: 49: 11.29$ & 0.45 & 9.3 & di & SB & 2.370 & 0.001 & VLT+Xshooter (J. Hjorth) \\
\hline
\end{tabular}

Notes.

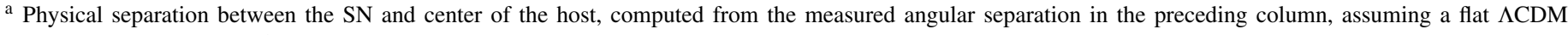
cosmology with $H_{0}=70, \Omega_{\mathrm{m}}=0.3$.

b Visual classifications for host galaxy morphology: $\mathrm{s}=$ spheroid, $\mathrm{d}=$ disk, $\mathrm{i}=$ irregular.

c Template-matching classification of host galaxy SED: $\mathrm{P}=$ Passive, $\mathrm{A}=$ Active, $\mathrm{SB}=$ Starburst type.

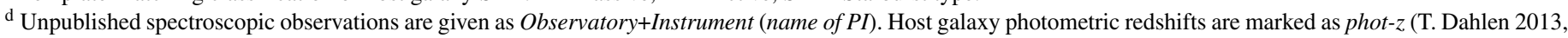
private communication).

To measure our SN detection efficiency and explore the associated systematic biases, we generated a catalog of 2,000 fake SNe. The catalog was drawn from a SNANA Monte Carlo simulation, such that the F160W magnitudes fill out a uniform distribution covering the range $21<m_{\mathrm{H}}<28$, and the $J-H$ colors were appropriate for Type Ia and Core Collapse SNe in the redshift range $0.1<z<2.8$. Each fake SN was then assigned to a "host galaxy" drawn from catalogs of extended sources in the CANDELS fields. The separation from the host galaxy center for each fake $\mathrm{SN}$ was then selected randomly from a normal distribution centered on 0 with a standard deviation of $2 \times R_{50}$, where $R_{50}$ is the radius of an aperture containing $50 \%$ of the host galaxy flux. This ensures that the fake SNe very roughly follow the distribution of host light (Kelly et al. 2008).

With magnitudes, colors, and positions defined, we generated synthetic PSFs for each fake SN using TinyTim, and planted 


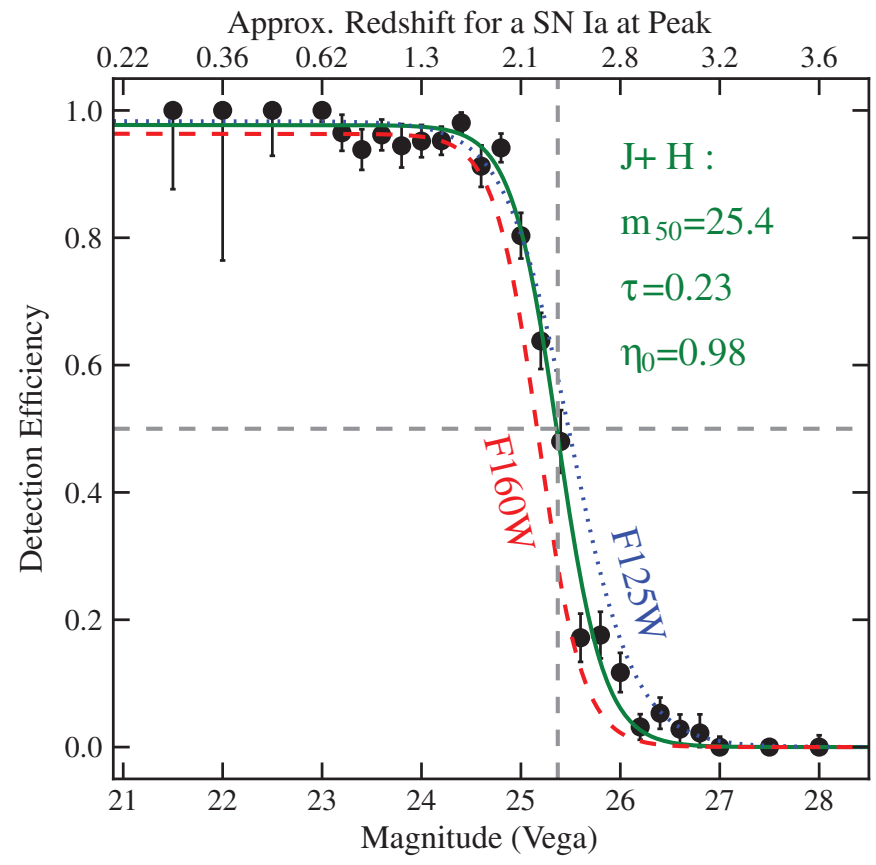

Figure 3. SN detection efficiency measurements as a function of magnitude in the " $J+H$ " band, taken as an average of the measured F125W and F160W magnitudes. Each point represents the fraction of fake $\mathrm{SNe}$ recovered by human searchers, with error bars indicating the standard deviation of the efficiency, computed using a Bayesian formalism (Paterno 2004). The bestfit model is shown as a solid (green) line, with best-fit parameters listed in the lower left. For reference, the equivalent best-fit curves for the $J$ and $H$ bands individually are shown in blue dotted and red dashed lines, respectively. The horizontal and vertical lines mark the $50 \%$ efficiency point for $J+H$ detections: $m_{50}=25.4 \mathrm{mag}$. The top axis marks the approximate redshift of a normal SN Ia with average extinction $\left(A_{V}=0.3\right)$ that would reach a peak brightness matching the $J+H$ magnitude on the bottom axis.

(A color version of this figure is available in the online journal.)

them in the FLT images, as described in Section 2.1. As the searchers reviewed each difference image, they were unaware of the number, brightness, and location of the fake $\mathrm{SN}$, so they recorded fake $\mathrm{SN}$ detections alongside detections of real $\mathrm{SNe}$. After completing each search, the fake SN detections (and nondetections) were used to calculate the recovery fraction.

Figure 3 shows the measured detection efficiency as a function of the " $J+H$ " magnitude: the average of the F125W and F160W magnitudes. We fit the efficiency measurements with a functional form similar to that used by Sharon et al. (2007), but we use only a single parameter to characterize the exponential turnover, and we allow for the peak efficiency to plateau at a value less than unity:

$$
\eta_{\text {det }}(m)=\eta_{0} \times\left(1+\exp \left(\frac{m-m_{50}}{\tau}\right)\right)^{-1},
$$

where $m$ is the apparent $J+H$ magnitude, $\eta_{0}$ is the maximum efficiency, $m_{50}$ is the magnitude at which the efficiency curve passes through the $50 \%$ line, and $\tau$ characterizes the exponential roll-off. The best-fit curve shown in Figure 3 has $m_{50}=25.4$, $\tau=0.23$, and $\eta_{0}=0.98$.

\subsection{Missing SNe in Galaxy Cores}

One concern for systematic bias entering into these detection efficiency measurements is the possibility that many $\mathrm{SNe}$ are obscured by difference imaging artifacts in the cores of bright

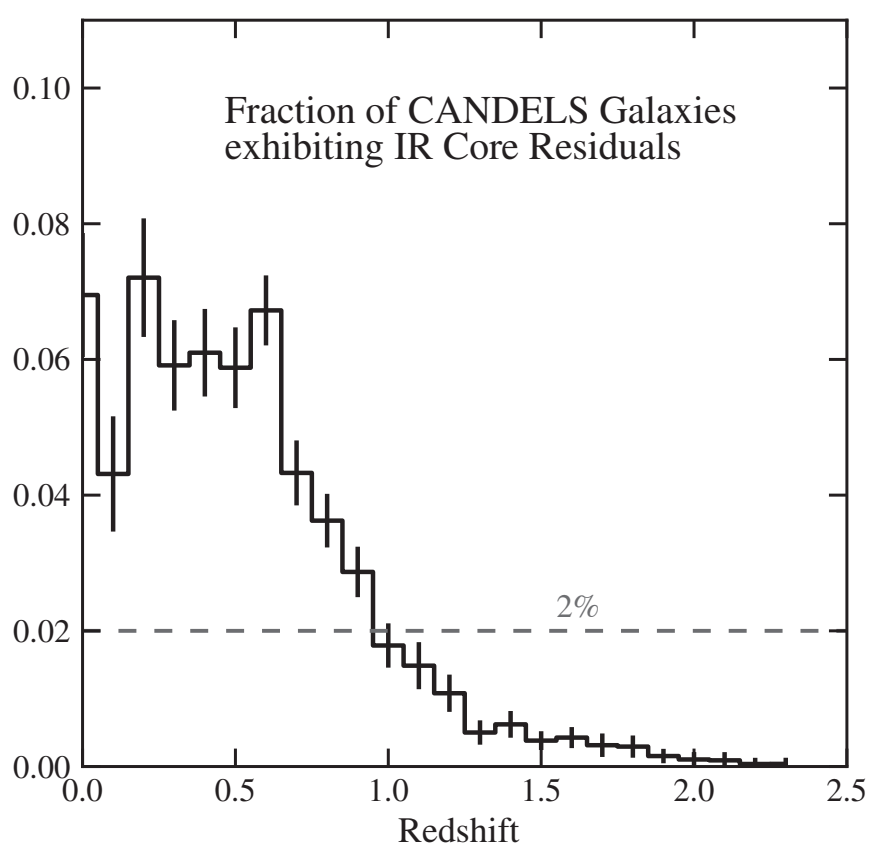

Figure 4. Fraction of CANDELS galaxies showing IR core residuals as a function of redshift, as determined from visual inspection of difference images generated by the $\mathrm{SN}$ data processing pipeline.

galaxies. The shot noise in these bright pixels is naturally higher than in the outskirts, as photon counts are elevated in both the search epoch and the template. Additionally, minor crossepoch registration errors can result in some residual flux in galaxy cores. In the CANDELS survey data these effects are both exacerbated by the under-sampled PSF of our single-epoch WFC3-IR images, as we have only two dithers per filter.

As shown in Figure 3, we have measured our maximum detection efficiency $\eta_{0}$ to be less than unity even for very bright $\mathrm{SNe}$, due to the fake $\mathrm{SNe}$ that happen to land in the noisy cores of bright galaxies. Our SN rate measurements will therefore naturally account for a small fraction of SNe that are missed in this manner. However, this built-in correction is only valid if the distribution of positions for the fake $\mathrm{SNe}$-relative to their host galaxy cores-is closely matched to the true distribution of the SN Ia population. Furthermore, it requires that the galaxies chosen for "hosting" our fake SNe are themselves representative of the population of SN Ia hosts. Our fake SN procedures were designed to meet these requirements at low and intermediate redshifts, but this does not necessarily carry over into the new high- $z$ regime.

To evaluate whether this effect might be introducing a strong bias at high- $z$, we visually inspected the CANDELS IR difference images and identified all galaxies that exhibited strong residuals. For each galaxy we tabulated the spectroscopic redshift or the best available photo- $z$ from CANDELS catalogs. Comparing this redshift distribution for core residuals against the count of all galaxies as a function of redshift gives us a measure of the fraction of (detected) galaxies that might obscure $\mathrm{SNe}$ in their bright cores. As shown in Figure 4, the fraction is less than $\sim 10 \%$ for all redshifts above 0.01 , and less than $\sim 2 \%$ for $z>1$-consistent with the value of $\eta_{0}$ measured from fake $\mathrm{SNe}$. This result suggests that any systematic bias from galaxy core residuals is very minor. Therefore, in the rate calculation we do not include any bias correction, and we do not add any contribution to the systematic uncertainty budget. 


\section{CLASSIFICATION}

To reach the final classification probabilities listed in Table 3 (and Table 9), we used a Bayesian analysis of the observed multicolor light curves. This photometric classification approach was used for our full sample of $65 \mathrm{SNe}$, supplemented by spectroscopic evidence for six objects, as described in Section 6. An early version of this classifier was introduced in Jones et al. (2013) with the presentation of SN UDS10Wil. Here we will again briefly describe the classification procedure, emphasizing recent changes.

\subsection{The STARDUST Classifier}

Our photometric classification approach uses SNANA to generate simulations of SN Ia and CCSN light curves. The SN Ia simulations use the SALT2 model (Guy et al. 2010), which has free parameters for the date of peak $\left(\mathrm{MJD}_{\mathrm{pk}}\right)$, redshift $(z)$, shape $\left(x_{1}\right)$, and color $(\mathcal{C})$. The simulated CCSNe are drawn from the SNANA library of 42 CCSN light curve templates (26 Type II and 16 Type $\mathrm{Ib} / \mathrm{c}$ ). These templates are derived from the SN samples of the Sloan Digital Sky Survey (Frieman et al. 2008; Sako et al. 2008; D'Andrea et al. 2010), Supernova Legacy Survey (Astier et al. 2006), and Carnegie Supernova Project (Hamuy et al. 2006; Stritzinger et al. 2009; Morrell 2012). Each CCSN template defines the underlying shape and color of the synthetic light curves, which is then modified with free parameters for the date of peak, redshift, host extinction $\left(A_{V}\right)$, and luminosity $(\Delta \mathrm{m}$, the shift in magnitudes relative to the peak of the assumed luminosity function). For this work, we fix the SALT2 model parameters $\alpha=0.135$ and $\beta=4.1$ (Scolnic et al. 2014), and for all simulated CCSNe we fix the extinction law to $R_{V}=3.1$.

Comparing these synthetic SNe to the observed light curves, we compute a likelihood using the $\chi^{2}$ statistic: $P\left(\mathbf{D}_{\mathrm{LC}} \mid \theta\right.$, Ia $) \propto$ $\exp \left(-\chi^{2} / 2\right)$, where the vector $\mathbf{D}_{\mathrm{LC}}$ is the observed $\mathrm{SN}$ light curve and the vector $\theta$ gives the parameter values for each realization of the SNANA models. We then apply priors for each model parameter (see Graur et al. (2014) for a detailed description of these priors) as well as a redshift-dependent prior for the fraction of SNe that are Type Ia: $P(\mathrm{Ia}, z)$ (see Section 34.2 below). Finally, we derive the total posterior probability that each object is an $\mathrm{SN}$ Ia, $P\left(\mathrm{Ia} \mid \mathbf{D}_{\mathrm{LC}}\right)$, by marginalizing over the nuisance parameters, $\theta$, and applying Bayes' theorem:

$$
P\left(\mathrm{Ia} \mid \mathbf{D}_{\mathrm{LC}}\right)=k_{\mathrm{LC}}^{-1} \int_{\theta} P(\mathrm{Ia}, z) P\left(\mathbf{D}_{\mathrm{LC}} \mid \theta, \mathrm{Ia}\right) d \theta
$$

The normalization factor $k_{\mathrm{LC}}$ is defined by requiring that that posterior probabilities for all three primary $\mathrm{SN}$ classes (Ia,Ib/c,II) sum to unity. The custom-built software package that executes this procedure is named STARDUST: Supernova Taxonomy And Redshift Determination Using SNANA Templates. The STARDUST code will be presented in full and publicly released in a subsequent paper (S. A. Rodney et al., in preparation).

There are two notable differences between the STARDUST classification procedure applied here and that described in Jones et al. (2013). First, in this work we do not use a free parameter for flux scaling, ${ }^{29}$ so the absolute values of the simulated $\mathrm{SN}$ fluxes are defined by the SN luminosity functions and

\footnotetext{
29 The $A$ in Equation (1) of Jones et al. 2013.
}

cosmology $\left(\Omega_{\mathrm{m}}=0.3, \Omega_{\Lambda}=0.7, w=-1\right)$ that are assumed in the SNANA simulations. To allow for some uncertainty in this baseline cosmology (or equivalently, introducing some increased scatter in the assumed SN luminosity functions) we include a non-zero model uncertainty term in the $\chi^{2}$ calculation. ${ }^{30}$ This is fixed at $8 \%$ of the simulated flux for SN Ia models and $10 \%$ for all CCSN models. Secondly, when the SN in question does not have a precise redshift from host galaxy spectroscopy, we use the host galaxy's photometric redshift probability distribution (photo- $z$ PDF) as the redshift prior.

Column 4 of Table 3 (and Table 9) presents the final SN classification probabilities, which will be used in Section 7 for the SN Ia rate calculation. Figure 5 (and Figures 15-17) shows the maximum likelihood light curve fit for each $\mathrm{SN}$, along with the associated best-fit model parameters. As described below, the systematic uncertainties associated with each classification probability are determined by varying two key priors that are not tightly constrained by observations: the assumed fraction of SNe that are of Type Ia and the distribution of host galaxy extinction.

\subsection{The Class Prior}

As with any Bayesian classification approach, the STARDUST classifier requires an input prior that quantifies the expectation that any given SN is of Type Ia-before applying any information from the SN light curve. We first assume that our sample is composed entirely of "normal" SNe, meaning that we assume no contamination from any other transient sources. This is a fairly safe assumption: AGNs and variable stars are excluded by our discovery requirements, under-luminous $\mathrm{SNe}$ like the .Ia (Bildsten et al. 2007) or Iax SNe (Foley et al. 2013) are well below our detection threshold, and super-luminous $\mathrm{SNe}$ (Gal-Yam 2012) have an intrinsic rate that is lower than that of normal SNe by a factor of about $10^{4}$ (Quimby et al. 2011).

We then define a redshift-dependent class prior $P(\mathrm{Ia}, z)$ as the fraction of all normal SNe at any given redshift $z$ that are Type Ia. Figure 6 shows the models used to define this prior and the associated systematic uncertainty. The baseline model (green curve) is anchored at $z=0$ by the measured Ia fraction (Smartt et al. 2009; Li et al. 2011), and then evolves at higher redshifts by following simple rate functions that match measured $\mathrm{SN}$ rates and theoretical expectations.

A more complete statistical treatment would define a large number of plausible models for the fraction of SNe that are SNe Ia, assigning each an appropriate weight based on current observations, and then marginalize over those many discrete priors to get a posterior probability that is not uniquely guided by the single choice of a baseline model. That approach is computationally expensive and will require further refinement of the STARDUST classifier. For this work, we have chosen to treat this choice of prior as a component of our systematic uncertainty budget. We take the baseline prior described above as our mid-rate model and then define two more models, labeled the high- and low-rate priors. These two respectively maximize and minimize the fraction of $\mathrm{SNe}$ that are assumed to be of Type Ia at any given redshift, and are shown in Figure 6. These bounding models offer a conservative estimate of the systematic uncertainty, because they are at the extreme limit of plausibility (if either were correct it would imply that the constraints from past rate measurements were all systematically wrong by more than $2 \sigma$ ).

${ }^{30} \sigma_{\text {sim }}^{2}$ in Jones et al. 2013. 

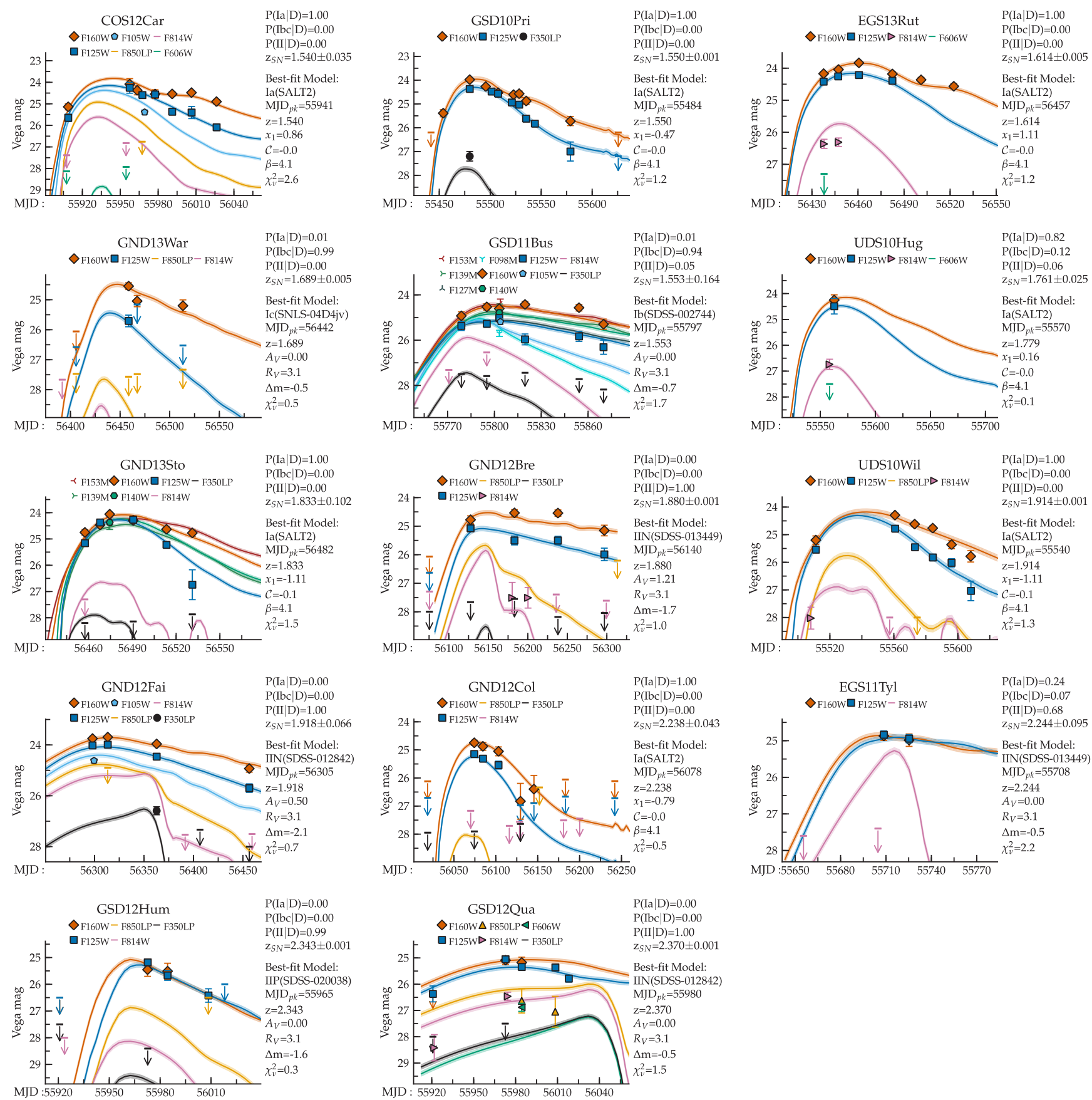

Figure 5. Light curves of $14 \mathrm{SN}$ with $z>1.5$. Each panel shows the observed photometry (in Vega mags) from CANDELS imaging with filled points and arrows for $3 \sigma$ upper limits. Error bars are typically less than the size of the points. Curves depict the maximum probability light curve fit for the most probable SN class, as determined by STARDUST. Classification probabilities and redshift as determined by STARDUST are listed on the right side of each panel, along with the parameters of the single model that delivers the highest posterior probability. Light curves for SN at $z<1.5$ are shown in Appendix B.

One might be concerned about the apparent circularity of using a redshift-dependent $P(\mathrm{Ia}, z)$ prior based on measured SN Ia rates in the service of a new SN Ia rate measurement. However, the bounding assumptions for our classification prior should ensure that our systematic uncertainty estimates account for this. To test that assertion, in Appendix A we evaluate an alternative prior that is based on the $\mathrm{SN}$ host galaxies and does not evolve with redshift. Tables 3 and 9 record the resulting STARDUST classifications using this modified prior as $P\left(\mathrm{Ia} \mid D_{\text {host }}\right)$ in Column 5. Table 8 reports the final effect of this prior switch on the observed count of SNe Ia and the volumetric rates.

\subsection{Host $A_{V}$ Distribution}

Another prior that can strongly affect the final classification probabilities is the assumed distribution of host galaxy extinctions, $P\left(A_{V}\right)$. As with the $\mathrm{SN}$ Ia fraction prior, we employ a baseline assumption (our mid-dust model) and two bounding assumptions (high-dust and low-dust) to constrain the possible systematic bias.

In keeping with observations, our dust models assume that the CCSN population suffers from significantly more dust extinction than the SN Ia population, at all redshifts (e.g., Smartt et al. 2009; Drout et al. 2011; Kiewe et al. 2012; Mattila 


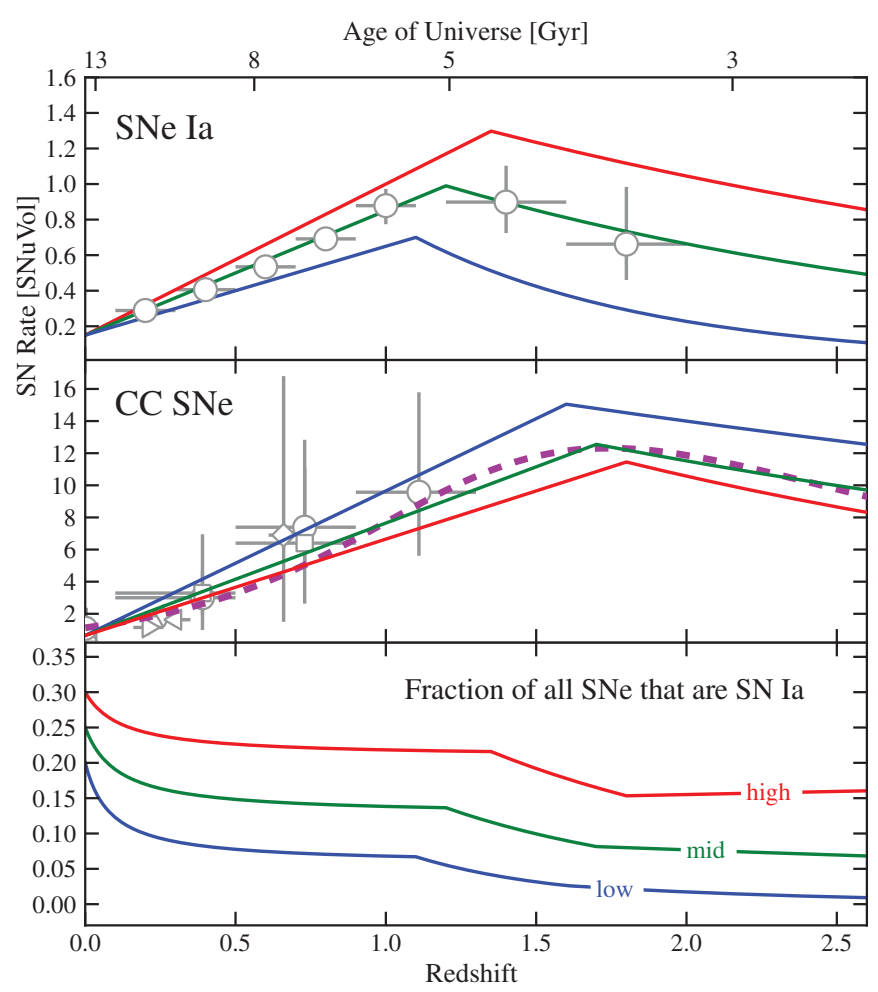

Figure 6. Deriving the redshift-dependent prior for SN class fractions. The top panel shows SN Ia rates and the middle panel shows CCSN rates. Both have observed rates plotted as open symbols. In the Ia case these are average values from all non-redundant field SN surveys. The CCSN rate points are the collection from Dahlen et al. (2012). In each panel the overlaid solid lines show three versions of a simple empirical model for the $\mathrm{SN}$ rates, and in the $\mathrm{CC}$ panel the magenta line traces the cosmic star formation history (see the text for details). The bottom panel plots the fraction of all SN explosions that are of Type Ia, derived from pairs of curves drawn from the top two panels and anchored to $25 \pm 5 \%$ at $z=0$ (Smartt et al. 2009; Li et al. 2011). These relative rate assumptions provide the high-, mid-, and low-rate priors that are used to derive classification probabilities and associated uncertainties for observed SNe.

(A color version of this figure is available in the online journal.)

et al. 2012). Our three dust models are generated from the positive half of a Gaussian distribution centered at $A_{V}=0$ with dispersion $\sigma$, plus an exponential distribution of the form $e^{-A_{V} / \tau}$. The parameter $\mathcal{R}_{0}$ gives the ratio of the height of the Gaussian to the height of the exponential, at $A_{V}=0$. The defining parameters and the expectation values for these three distributions are summarized in Figure 7.

When simulating SN Ia with the SALT2 model, the SN color is defined by the SALT2 $\mathcal{C}$ parameter. This color term comprises both the intrinsic SN color as well as reddening from host galaxy dust. The distribution of $\mathcal{C}$ values can therefore be described as a convolution between a narrow Gaussian (the intrinsic dispersion of SN Ia colors) and a function describing the distribution of host galaxy extinctions. Following Barbary et al. (2012) and Scolnic et al. (2014), we approximate the $A_{V}$ distributions of previous SN Ia studies by modifying the red side of the SALT2 $\mathcal{C}$ distribution so that the simulated $\mathrm{SN}$ colors match the output of that convolution.

Specifically, our high-dust model for SN Ia matches the baseline $A_{V}$ distribution used by Neill et al. (2006): a Gaussian with $\sigma=0.62$. The mid-dust model is equivalent to the exponential distribution of Kessler et al. (2009a): $P\left(A_{V}\right)=$ $e^{-A_{V} / \tau}$, with $\tau=0.33$. Our low-dust model for SN Ia assumes minimal dust extinction, using a narrow Gaussian with $\sigma=0.15$ plus a shallow exponential with $\tau=0.15$. A more complete

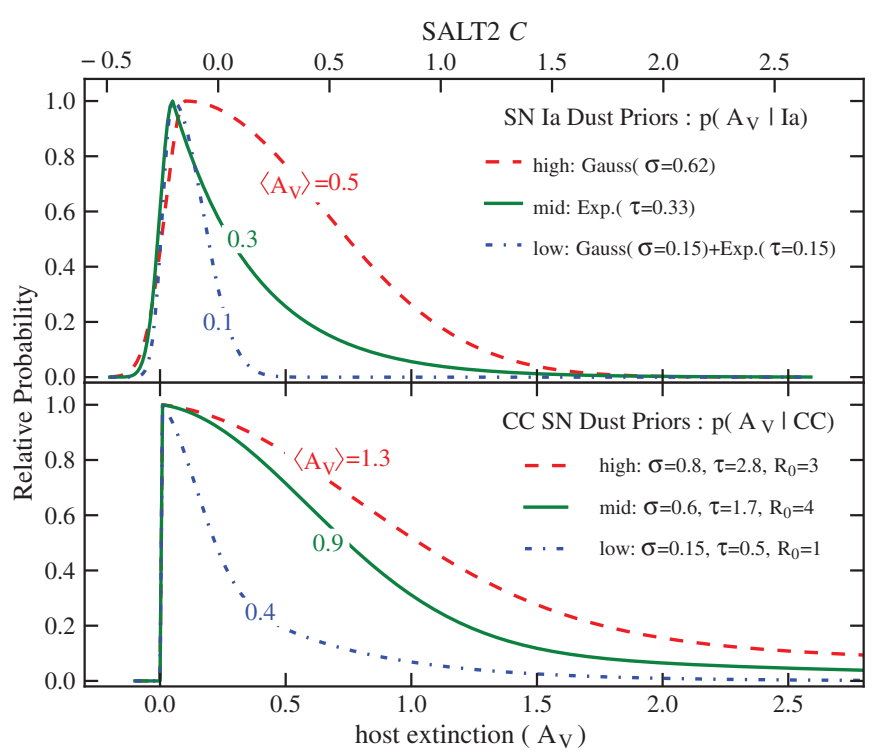

Figure 7. Prior probability distributions for the SN host galaxy extinction, as used in the STARDUST classifier code. The top panel shows the three priors applied to the SN Ia models, and the bottom panel shows the equivalent priors used for CCSN models. In both cases the high-dust model is shown as a (red) dashed line, the mid-dust model as a (green) solid line, and the low-dust model as a (blue) dash-dotted line. Each model is composed of a Gaussian and/or an exponential function (see the text for details) and the parameters for those components are listed in the legends. Each curve is labeled with its expectation value $\left\langle A_{\mathrm{V}}\right\rangle$, giving the "weighted average" of the host galaxy extinction for that model.

(A color version of this figure is available in the online journal.)

treatment of host galaxy dust would include a prescription for the redshift dependence of these extinction distributions, as $\mathrm{SN}$ hosts are expected to be dustier at redshifts approaching $z=2$ (Mannucci et al. 2007). As we will see in Section 7, this systematic uncertainty is not a dominant component of the error budget, so redshift dependence is left for future work.

To determine the combined systematic effects from the SN Ia fraction prior and the dust assumptions, we compute each SN classification probability nine times: three for each $\mathrm{SN}$ rate prior $\times$ three for each dust model. The mid-rate + middust combination gives us our baseline classification probability, which dictates how much each individual SN contributes to the total count of observed SN Ia, $N_{\text {obs }}$. The extrema from this set of nine probabilities then provide the systematic classification errors, which propagate directly into the systematic uncertainty on the $\mathrm{SN}$ Ia rate.

\subsection{STARDUST Validation Test}

A full investigation of the accuracy of the STARDUST classification code is beyond the scope of this paper. It is useful, however, to examine a simple validation test to demonstrate that this classifier is not grossly biased or ineffective. To that end, we have applied the STARDUST classifier to the "Gold" sample of $31 \mathrm{SNe}$ from the GOODS and PANS surveys (Strolger et al. 2004; Riess et al. 2007) that have spectroscopic classifications. These surveys were carried out using the HST Advanced Camera for Surveys (ACS), and share many of the survey design characteristics of the CANDELS SN program. STARDUST correctly classifies 29 of the $31 \mathrm{SNe}$ (93.5\%), using only their redshifts and photometric data. This demonstrates that we have a low false negative rate with STARDUST, i.e., we rarely misclassify a true SN Ia. Unfortunately, this validation test is 
not sensitive to false positives - true CCSNe being misclassified as Type Ia-because we only have a single spectroscopically confirmed CCSN in this Gold sample. Preliminary testing of the STARDUST classifier using simulated SNe suggests that the Ia sample purity for photometrically classified SNe could be on the order of $95 \%$ (these validation tests will be presented in a future paper).

\section{HOST GALAXIES}

Host galaxy information is recorded in Table 4 for the SNe at $z>1.5$, and in Table 10 for the low redshift SNe. As can be seen in Figure 2 (and Figures 13-14), most of the 65 CANDELS SNe can be unambiguously associated with a host galaxy, because the host is isolated, or the $\mathrm{SN}$ is clearly embedded within the stellar light of a single galaxy. There are, however, a few exceptions.

For SN COS12Her, there are two host galaxy candidates: the nearest and brightest has a photometric redshift of 0.403 ${ }_{-0.11}^{+0.04}$, but the observed SN colors cannot be adequately matched by any normal $\mathrm{SN}$ template in that redshift range. The second COS12Her host candidate has a photo- $z=1.10_{-0.19}^{+0.16}$. At this higher redshift, the STARDUST classifier finds a very good match to the observed light curve with a Type II-P template.

SN GND13Sto is separated by several arcseconds from all nearby galaxies. Of the six galaxies within $5 \operatorname{arcsec}$ of the SN position, five have a photo- $z$ distribution that peaks close to $z=1.8$, including one with a spectroscopic redshift from the Spitzer Infrared Spectrograph of $z=1.80 \pm 0.02$ (Murphy et al. 2009). This is suggestive of a small cluster or group of galaxies at that redshift, with SN GND13Sto possibly associated with a low surface brightness group member or tidal stream. Indeed, applying STARDUST to the well-sampled SN GND13Sto light curve (and allowing for a broad redshift range, $z=1.8 \pm 1.2$ ), we find the maximum likelihood match is an SN Ia template at $z=1.86 \pm 0.05$.

There are four SNe for which the host galaxy is barely detectable in the deep IR imaging mosaics from CANDELS. These are SNe GND12Kin, EGS11Nix, GND13Gar, and GSD11Bus. All four of these objects lack a clear spectroscopic redshift from their host, so we are limited to using photometric redshifts for the STARDUST priors. In all of these cases, with STARDUST we find good template matches within the allowed redshift range.

\subsection{Morphology and SED Type}

The SN host galaxies (along with all CANDELS galaxies) were classified visually by members of the CANDELS team into three morphological categories: spheroid, disk, and irregular. Visual classifications were performed using template images so that the presence of the SN did not bias the classification. Each galaxy can be assigned to multiple categories, so we also include two intermediate categories: spheroid+disk and disk+irregular. These morphological classes roughly correspond to broad bins over the Hubble sequence. This is appropriate for classifying galaxies at high redshift where distinguishing between, say, an $\mathrm{E}$ and an S0 galaxy is more difficult and less meaningful. Details of the CANDELS morphological classification procedure are presented in Kartaltepe et al. (2012).

We also record the "SED type" for each SN host galaxy, determined by matching the full galaxy SED against a set of templates, using the GOODZ code (Dahlen et al. 2010). The GOODZ template library is segregated into three groups, labeled according to the amount of ongoing star formation: passive (early type), active (late type), and starburst. We use the best- matching SED template for each CANDELS SN host galaxy to assign it to one of those bins.

For two of the SNe (COS12Car and GND12Daw) there is no discernible host at the location of the SN and no nearby galaxy presents a plausible host candidate. For both of these objects we do have spectroscopic redshift information from the SNe themselves, as detailed in Section 6. For the other 63 $\mathrm{SNe}$ in our sample, 10 host galaxies are classified as spheroids, 15 as spheroid+disk, 17 as disk, 7 as disk+irregular, and 8 as irregular. For six of our SNe, the host galaxy is detected, but is too faint for reliable visual classification, so we report the host morphology as "unclassifiable." For the 63 objects with detectable host galaxies we have 2 passive, 24 active, and 37 starburst-like SEDs.

\section{GRISM SPECTROSCOPY}

There are six objects in our sample for which we collected useful HST grism spectroscopy of the SNe themselves. SN GSD10Pri, a Type Ia SN at $z=1.55$, was presented in Rodney et al. (2012) with an analysis of the host galaxy in Frederiksen et al. (2012). SN UDS11Wil, a Type Ia SN at $z=1.91$, was described in Jones et al. (2013). Figure 8 presents grism spectra for the remaining four: SNe GSD11Was, GND12Daw, GND13Gar, and COS12Car. In all of these cases the signal-tonoise ratios and rest-frame wavelength coverages are insufficient for a purely spectroscopic classification. Rather, as with GSD10Pri and UDS11Wil, we used the spectroscopic information to supplement the STARDUST photometric classifier, leading to a more robust classification.

The host galaxy of SN GSD11 Was has a photometric redshift of $z=1.04 \pm 0.3$. We obtained a spectrum of SN GSD11Was with the WFC3-G141 grism, shown in Figure 8 (top left). Here we can see hints of an absorption feature at $\sim 14000 \AA$. At a redshift of $z \sim 1.3$ this feature can be explained as the characteristic Si II absorption trough seen at rest-frame $\sim 6150 \AA$ in SN Ia spectra. Photometric classification of this $\mathrm{SN}$ with STARDUST agrees, finding the object is best matched by an SN Ia template at $z=1.3 \pm 0.05$. (see the light curve plot in Appendix B, Figure 17).

SNe GND12Daw, GND12Gar, and COS12Car all have no detectable host galaxy in any optical or NIR band, and no neighboring galaxies have redshifts that allow for acceptable light curve template matches in STARDUST. The most likely explanation is that these $\mathrm{SNe}$ reside in very low surface brightness galaxies, too faint for detection even in our deep HST imaging.

The spectrum for GND12Daw shows hints of a broad emission feature at $12000 \AA$, which could be $\mathrm{H} \alpha$ emission, if the object is at $z=0.830$. This could be interpreted as strong Balmer line emission from an otherwise very faint host galaxy, or it could be showing the $\mathrm{H} \alpha$ emission from the SN itself-characteristic of Type II-P spectra. Given the very low signal-to-noise ratio in this spectrum (it was derived from just a single orbit of HST observations), this alone would be weak support. However, when allowing STARDUST to search over a redshift range $0.1<z<2.0$, we find that a Type II-P light curve template consistently provides the strongest match to the broad light curve shape of this $\mathrm{SN}$, and the solution at $z \sim 0.8$ provides $>90 \%$ of the total likelihood.

For GND12Gar (upper right) the absorption trough at $\sim 7700 \AA$ provides a key observable that can anchor the fit and define the age of the SN. If this feature corresponds to Ca II absorption, then that would fix the object's redshift to 

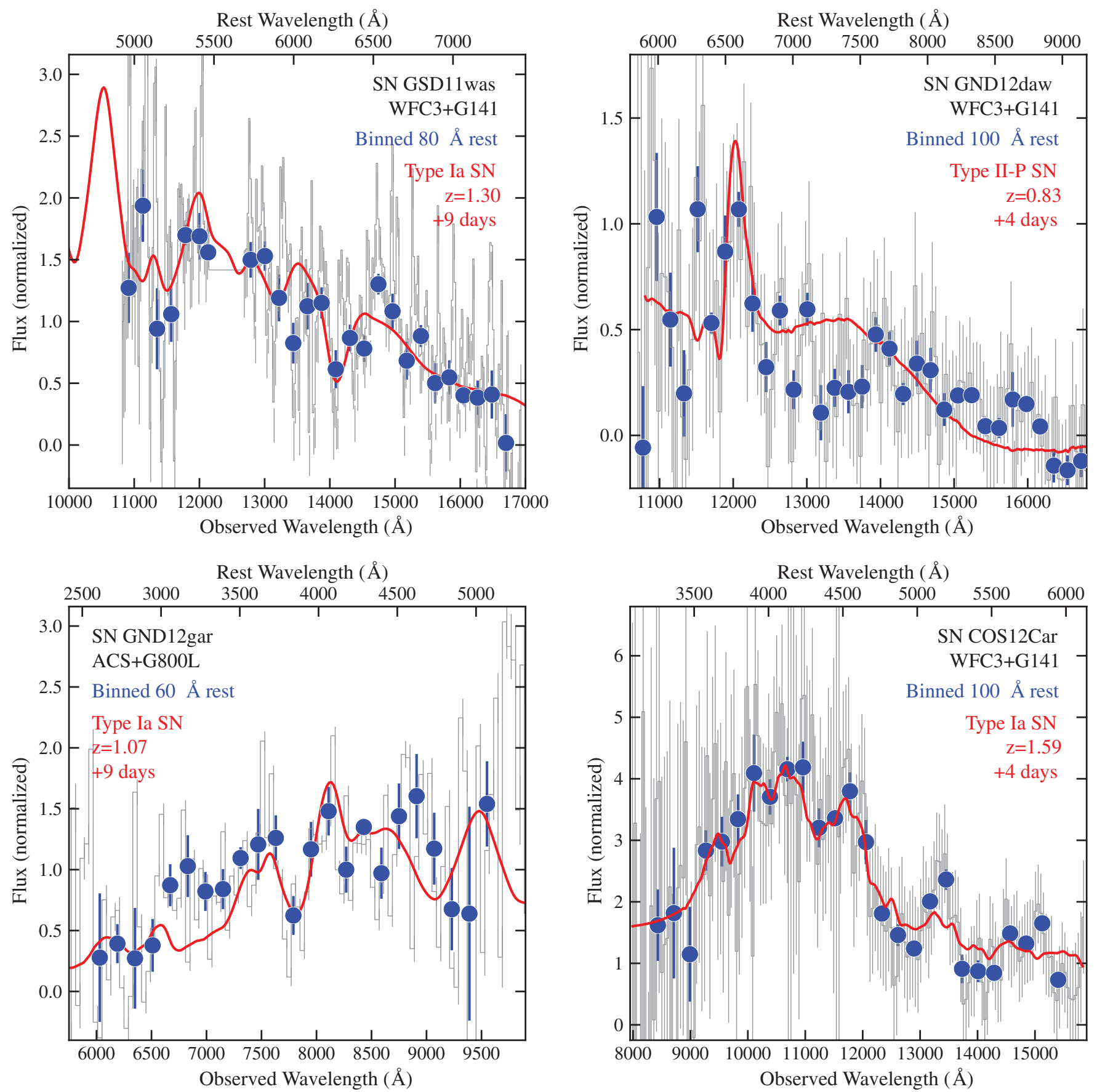

Figure 8. Observed spectra and spectral template matches for four CANDELS SNe. The spectra for GSD11Was (top left) and GND13Daw (top right) were collected using the G141 grism on HST's WFC3-IR detector. The GND13Gar spectrum (lower left) used the ACS G800L grism, and the spectrum of COS12Car (lower right) combines observations from both the G102 and G141 WFC3 grisms. In each panel the observed flux is shown in gray, with binned points overlaid in blue, and the best-fitting template spectrum in red.

$z=1.07 \pm 0.02$. At this redshift the light curve is matched very well by SN Ia templates, and no other redshift or SN class can provide a better light curve match.

The strongest spectral constraint for SN classification comes from SN COS12Car. For this object we have observations with both the WFC3 G102 and G141 grisms. Fitting the composite spectrum with the SuperNova IDentification (SNID) software (Blondin \& Tonry 2007), we find the best template match is a Type Ia SN at $z=1.59$. Once again we find that the STARDUST photometric classification agrees well with this spectroscopic information: an SN Ia light curve at $z \sim 1.6$ provides the best available light curve template.

\section{THE VOLUMETRIC SN Ia RATE}

To convert the observed SN counts into a volumetric rate, we use an approach similar to Dahlen et al. (2008) and Rodney \& Tonry (2010). We first divide up the detected SNe into four redshift bins of width $\Delta z=0.5$. The total contribution to the SN Ia count from each observed SN is equal to the Ia classification probability for that object. For objects with uncertain redshifts, this fractional contribution is distributed over multiple redshift bins according to the integrated area of the redshift PDF. Adding up all the fractional counts gives us the total observed SN Ia count as a function of redshift: $N_{\text {obs }}(z)$. Statistical 
Table 5

Observed SN Ia Counts and the Volumetric Rate

\begin{tabular}{|c|c|c|c|c|c|c|c|c|}
\hline \multirow[t]{2}{*}{ Redshift } & \multicolumn{3}{|c|}{ Observed Count $^{\mathrm{a}}$} & \multicolumn{2}{|c|}{ Control Count $^{b}$} & \multicolumn{3}{|c|}{ SN Rate ${ }^{c}$} \\
\hline & $N_{\mathrm{obs}}$ & $\delta N_{\text {Poiss }}$ & $\delta N_{\text {syst }}$ & $N_{\text {ctrl }}$ & $\delta N_{\text {syst }}$ & SNR & $\delta \mathrm{SNR}_{\text {stat }}$ & $\delta \mathrm{SNR}_{\text {syst }}$ \\
\hline 0.25 & 1.46 & $\begin{array}{l}+2.46 \\
-1.06\end{array}$ & $\begin{array}{l}+0.48 \\
-1.44\end{array}$ & 4.10 & $\begin{array}{l}+0.01 \\
-0.04\end{array}$ & 0.36 & $\begin{array}{l}+0.60 \\
-0.26\end{array}$ & $\begin{array}{l}0.12 \\
-0.35\end{array}$ \\
\hline 0.75 & 7.19 & $\begin{array}{l}+3.80 \\
-2.62\end{array}$ & $\begin{array}{l}+1.94 \\
-2.54\end{array}$ & 14.11 & $\begin{array}{l}+0.53 \\
-1.71\end{array}$ & 0.51 & $\begin{array}{l}+0.27 \\
-0.19\end{array}$ & $\begin{array}{l}+0.23 \\
-0.19\end{array}$ \\
\hline 1.25 & 8.47 & $\begin{array}{l}+4.02 \\
-2.85\end{array}$ & $\begin{array}{l}+0.45 \\
-2.04\end{array}$ & 13.16 & $\begin{array}{l}+2.46 \\
-4.05\end{array}$ & 0.64 & $\begin{array}{l}+0.31 \\
-0.22\end{array}$ & $\begin{array}{l}+0.34 \\
-0.23\end{array}$ \\
\hline 1.75 & 5.54 & $\begin{array}{l}+3.49 \\
-2.28\end{array}$ & $\begin{array}{l}+0.17 \\
-0.61\end{array}$ & 7.67 & $\begin{array}{l}+3.47 \\
-2.99\end{array}$ & 0.72 & $\begin{array}{l}+0.45 \\
-0.30\end{array}$ & $\begin{array}{l}+0.50 \\
-0.28\end{array}$ \\
\hline 2.25 & 1.24 & $\begin{array}{l}+2.39 \\
-0.96\end{array}$ & $\begin{array}{l}+0.13 \\
-0.16\end{array}$ & 2.52 & $\begin{array}{l}+1.72 \\
-1.07\end{array}$ & 0.49 & $\begin{array}{l}+0.95 \\
-0.38\end{array}$ & $\begin{array}{l}+0.45 \\
-0.24\end{array}$ \\
\hline
\end{tabular}

Notes.

a Statistical uncertainties reflect the limits that contain $68 \%$ of the Poisson distribution. Systematic uncertainties are due to the assumed dust model and rates prior.

b Systematic uncertainties are due to the assumed dust model.

${ }^{c}$ The SN Ia rate measurements in units of SNuVol $=10^{-4} \mathrm{yr}^{-1} \mathrm{Mpc}^{-3} h_{70}{ }^{3}$.

uncertainties for each bin are defined by the points encompassing the central $68 \%$ of the Poisson distribution.

We then use Monte Carlo simulations to compute a "control count" for each bin, $N_{\text {ctrl }}(z)$, which is the expected number of $\mathrm{SNe}$ Ia that would be detected if the cosmic SN Ia explosion rate were constant for all redshifts at $1 \mathrm{SNuVol}=$ $10^{-4} \mathrm{yr}^{-1} \mathrm{Mpc}^{-3} h_{70}{ }^{3}$. By simulating SN Ia light curves within the context of the CANDELS survey, the computation of $N_{\text {ctrl }}(z)$ incorporates both the survey volume and the control time (the time interval over which any given $\mathrm{SN}$ is visible to our survey).

We again use SNANA as our simulation engine, this time generating 100,000 SNe Ia based on the SALT2 light curve model (Guy et al. 2010). The light curve for each synthetic SN is determined by a set of four variables: date of peak brightness $m j d_{\mathrm{pk}}$, redshift $z$, SALT2 shape parameter $x_{1}$, and SALT2 color parameter $\mathcal{C}$. The $\mathcal{C}$ parameter in SALT2 includes both intrinsic $\mathrm{SN}$ color as well as reddening from host galaxy dust. Each redshift $z$ is drawn from the range $0<z<2.5$, following the constant volumetric rate assumption. To translate this redshift into a luminosity distance, we use our baseline cosmology: $\Omega_{\mathrm{m}}=0.3, \Omega_{\Lambda}=0.7, w=-1, H_{0}=70$. Values for $x_{1}$ are drawn from a normal distribution with mean and dispersion from Kessler et al. (2009a): $\bar{x}_{1}=-0.13, \sigma_{x_{1}}=1.24$. The color parameters are draw from a bifurcated Gaussian distribution with $\overline{\mathcal{C}}=0.04, \sigma_{\mathcal{C}}^{-}=0.08$, and $\sigma_{\mathcal{C}}^{+}=0.25$-parameters that match the mid-dust model described in Section 4.3 and Figure 7.

To choose values for $m j d_{\mathrm{pk}}$ we first establish the width of the survey window at any given redshift. By examining simulated $\mathrm{SN}$ Ia light curves in F125W and F160W, we find $t_{\min }$ and $t_{\max }$, the minimum and maximum dates relative to peak for which each SN would be detectable to our survey. Here, detectability is defined by measuring the change in flux relative to a template epoch 52 days prior, and requiring that the corresponding $J+H$ magnitude is brighter than the $50 \%$ detection threshold seen in Figure 3 . The allowed range for the simulated $m j d_{\mathrm{pk}}$ values at redshift $z$ is then $\left[\mathrm{MJD}_{\text {first }}-t_{\max }(z), \mathrm{MJD}_{\text {last }}+t_{\min }(z)\right]$, where $\mathrm{MJD}_{\text {first }}$ and $\mathrm{MJD}_{\text {last }}$ are the epochs for the first and last search epoch, respectively. For each redshift, random $m j d_{\mathrm{pk}}$ values are then drawn from a flat distribution spanning this survey window.

Each synthetic SN is "observed" in the SNANA simulator using survey parameters that match the actual operations of the CANDELS program, as given in Tables 1 and 2. For the wide fields (COSMOS, EGS, UDS, and the wings of the GOODS fields) we only have a single search epoch, so these fields are simulated together as the "CANDELS-Wide" search field. The
10 epoch GOODS-S and GOODS-N Deep fields are treated separately, but all observational parameters are computed in the same way. Due to the excellent stability of the HST photometric system, we adopt a single set of average values for zero points and detector noise. The total area in each field reflects the area in which SN searching can be done, i.e., the area covered by the SN search epoch and at least one prior epoch. The cadence between epochs is nominally 52 days, but the actual separation in time varies from pointing to pointing due to HST scheduling constraints. For this simulation we use a mean cadence for each field and each epoch, weighted by the area available for SN searching. Finally, we use the detection efficiency curve of Figure 3 to define the probability of "detecting" each simulated $\mathrm{SN}$ in any given epoch.

Counting the number of detected synthetic $\mathrm{SNe}$ in each redshift bin gives us the control count, which carries units of $\mathrm{SNuVol}^{-1}$. The observed volumetric rate of $\mathrm{SN}$ Ia explosions is simply the ratio

$$
\operatorname{SNR}_{\mathrm{Ia}}(z)=\frac{N_{\mathrm{obs}}(z)}{N_{\mathrm{ctrl}}(z)} .
$$

The measured values for $N_{\text {obs }}(z), N_{\text {ctrl }}(z)$, and $\operatorname{SNR}(z)$ from the CANDELS survey are given in Table 5 along with uncertainty estimates due to statistical noise (Poisson errors) and systematic biases. The total sample size is quite small, with only $\sim 21 \mathrm{SNe}$ Ia across all redshifts and fewer than seven in each bin. This means that the statistical errors are substantial, roughly equal to, or greater than the systematic uncertainties in every redshift bin. One cannot infer a clear trend with redshift from these data alone, but rather we must evaluate them within the context of other rates measurements and SN Ia progenitor models.

\subsection{Systematic Uncertainties}

In preceding sections we considered three principal sources of systematic biases: (1) missing $\mathrm{SN}$ detections due to subtraction artifacts in the cores of bright galaxies, (2) the assumed fraction of SNe that are of Type Ia as a function of redshift, and (3) the assumed distribution of host galaxy dust extinction values. We have determined that bias from the first source is negligible. The second is examined in more detail in Appendix A, and is reflected in the systematic uncertainty estimates for the count of observed SNe Ia (Column 4 of Table 5). The third item also affects the control count (Column 6).

Other potential sources of systematic bias include: errors in the luminosity functions for SN subclasses, biases in the SN Ia 


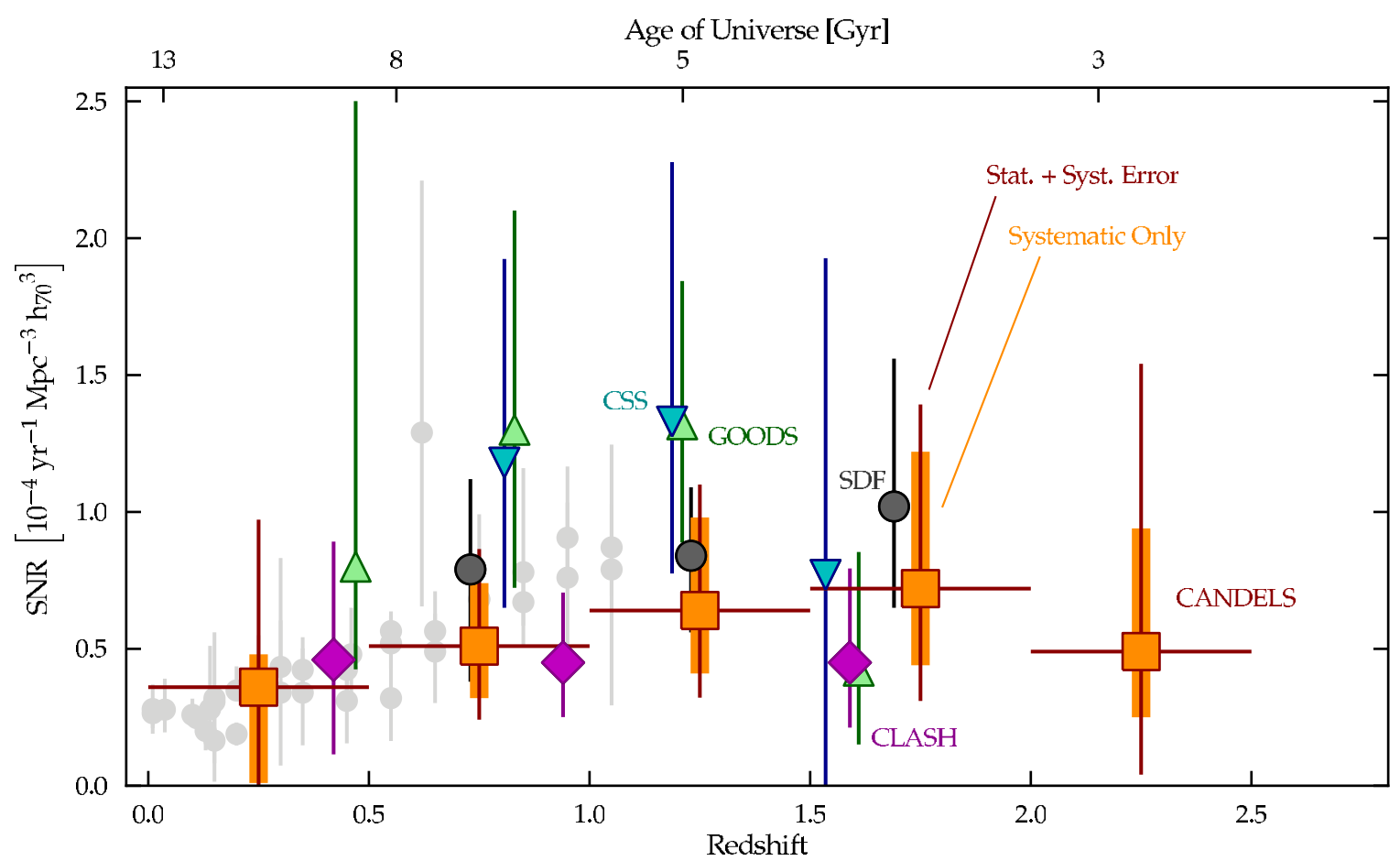

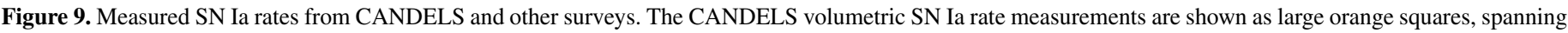

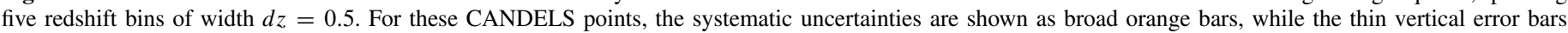

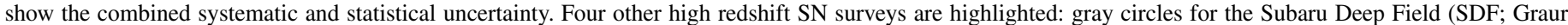

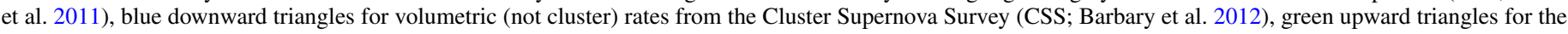

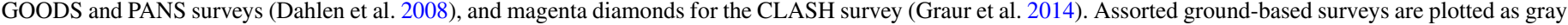
circles, as in Figure 1.

model or the CCSN template libraries, and peculiar detection biases from individual human searchers. For this work, these contributions to the systematic error budget are assumed to be insignificant. Future analysis with the full CANDELS+CLASH sample will revisit this assumption and evaluate these systematic error sources.

\section{TESTING SN Ia PROGENITOR MODELS}

The measurement of volumetric SN Ia rates at high redshift is principally motivated by two astrophysical investigations. First, it directly informs our understanding of the cosmic enrichment history, as SNe Ia are a primary source for Fe group elements in the universe (e.g., Wiersma et al. 2011). Second, by measuring the delay between star formation and SN explosion through the DTD formalism, one can draw inferences about the nature of SN Ia progenitor systems. In this work we limit our discussion to the latter, beginning with a comparison to other published SN Ia rates, then evaluating new constraints on progenitor models, and finally making a projection toward future improvements.

\subsection{Comparison to Earlier Rate Measurements}

Figure 9 presents the CANDELS SN Ia rates within the context of other rate measurements from the literature. The CANDELS rate measurements are shown in five bins of width $\Delta z=0.5$ as large orange squares. Rate measurements from 13 ground-based surveys are plotted as small gray circles, reaching out to $z=1.1$. Four surveys that previously extended the rate measurements to $z \sim 1.5$ are highlighted with larger colored points (see the caption for details).

As with past $H S T$ surveys, our survey volume is too small to add any useful new information at $z<1$, but the general agreement with ground-based surveys is an important validation that our rate measurements are realistic. For a more informative comparison, we turn now to the high- $z$ side of the plot.

Before CANDELS and CLASH, there were just three surveys with any $\mathrm{SN}$ Ia rate measurements above $z \sim 1.2$. First were the GOODS SN surveys, which used the HST ACS camera to measure the $\mathrm{SN}$ Ia rate to $z \sim 1.8$ (GOODS; Strolger et al. 2004; Dahlen et al. 2004). These data were interpreted as showing a flattening or a downturn in the $\operatorname{SNR}(z)$ at $z>1.2$, a trend that garnered support from additional HST observations and independent analyses (Dahlen et al. 2008; Kuznetsova et al. 2008), including another HST+ACS program, the Cluster Supernova Survey (CSS; Barbary et al. 2012). ${ }^{31}$ Ground-based rate measurements from the Subaru Deep Field (SDF) survey also reached out to $z \sim 1.8$, though these were more susceptible to systematic biases due to the absence of light curve information and spectroscopy for SN classification (Poznanski et al. 2007; Graur et al. 2011). As the CANDELS and CLASH surveys began, it was still an open question as to whether we had now seen the peak in the SN Ia rate, or if it was continuing to rise beyond $z \sim 1.2$.

The GOODS, CSS, and SDF surveys all used optical bands that correspond to rest-frame near-UV wavelengths at high redshift. For an $\mathrm{SN}$ at redshift $z \sim 1.5$, observations in the $z$ band ( $\sim 9000 \AA$ ) are sampling the rest-frame $U$ band, while the observer's $i$ band $(\sim 8000 \AA$ ) reaches well into the rest-frame near-UV. At these wavelengths the available SN light curve templates for use in photometric classification are poor, because most nearby SN surveys do not observe SNe in the UV. Both

\footnotetext{
31 This was a survey of galaxy clusters, but the work of Barbary et al. (2012)
} presented volumetric SN Ia rates from the $\mathrm{SNe}$ detected outside the clusters. 
SNe Ia and CCSNe also exhibit more natural heterogeneity at these blue wavelengths, and this is all compounded by a greater sensitivity to dust obscuration in the UV. Thus, optical-wavelength surveys were more susceptible to both of the components that dominate the systematic uncertainties of high- $z$ SN Ia rate measurements: classification bias and dust obscuration. By contrast, the CANDELS survey utilized $J$ and $H$ infrared bands that sample rest-frame optical wavelengths, even out to redshift $z \sim 2.5$. The CANDELS rates should therefore be less strongly affected by those systematic biases.

At $z=1.25$ the CANDELS rate is substantially lower than all past measurements, though still consistent at the $1-2 \sigma$ level. The CANDELS rate then climbs slightly in the bin at $z=1.75$, where it is completely consistent with past measurements. CANDELS is the only survey with any detections beyond $z=2$, and there we have only a single object with a strong probability of being an SN Ia (SN GND12Col in the GOODS-N field, at $z \sim 2.24$ ). The rate formally shows a decline to $z=2.25$, although this change is much smaller than the uncertainties. The CANDELS rates are fully consistent at all redshifts with the similarly derived rates from CLASH, which are also quite low relative to past surveys (Graur et al. 2014).

Due to the small sample sizes and large uncertainties, none of these individual high- $z$ surveys has sufficient precision to clearly delineate the shape of the $\operatorname{SNR}(z)$ curve. From Figure 9 we can only say that the $\mathrm{SN}$ Ia rate rises steadily to $z \sim 1.2$, and then is flat or slowly declining at redshifts $z>1.2$.

Each independent analysis of SN Ia rates makes slightly different assumptions about host galaxy extinction and each takes a different approach to SN classification. These differences become particularly important at $z>1$ where observed rates are dominated by HST SN surveys, which have much less complete spectroscopic information. Here the potential for systematic biases is much greater, as a larger fraction of SN classifications and host galaxy redshifts rely on photometric data alone.

An optimal approach would be to treat the past decade of $H S T$ SN surveys as a single composite survey. One could compute the rates from all the HST SN surveys together, using the same SN classifier(s), consistent models for (redshift-dependent) host galaxy extinction, and the best available host galaxy redshift information. Such an effort is beyond the scope of this work, but will be an important contribution for future DTD tests.

\subsection{Isolating the Prompt SN Ia Fraction}

To examine how the observed SN Ia rate can inform the modeling of SN Ia progenitors, we will employ a simple toy model, motivated by a variety of recent observations and theoretical predictions. For a complementary analysis using DTD predictions from binary population synthesis modeling, see Graur et al. (2014). Multiple lines of evidence now suggest that the overall shape of the SN Ia DTD follows a $t^{-1}$ power law for times $t>500 \mathrm{Myr}$ (see Maoz \& Mannucci 2012, for a recent review). At short delays, $t<500 \mathrm{Myr}$, the evidence is much less definitive, and this is the region where the CANDELS observations may provide unique new insight. Thus, our primary question is: what fraction of SNe Ia explode within $500 \mathrm{Myr}$ of their formation?

To isolate this "prompt SN Ia fraction," we define a bifurcated DTD model: the long-delay component follows a $t^{-1}$ distribution for all times $t>500 \mathrm{Myr}$, and the prompt component is set to be constant with time for $t<500 \mathrm{Myr}$, down to a lower limit of $t_{\min }=40 \mathrm{Myr}$ (the shortest possible time to reach explosion;

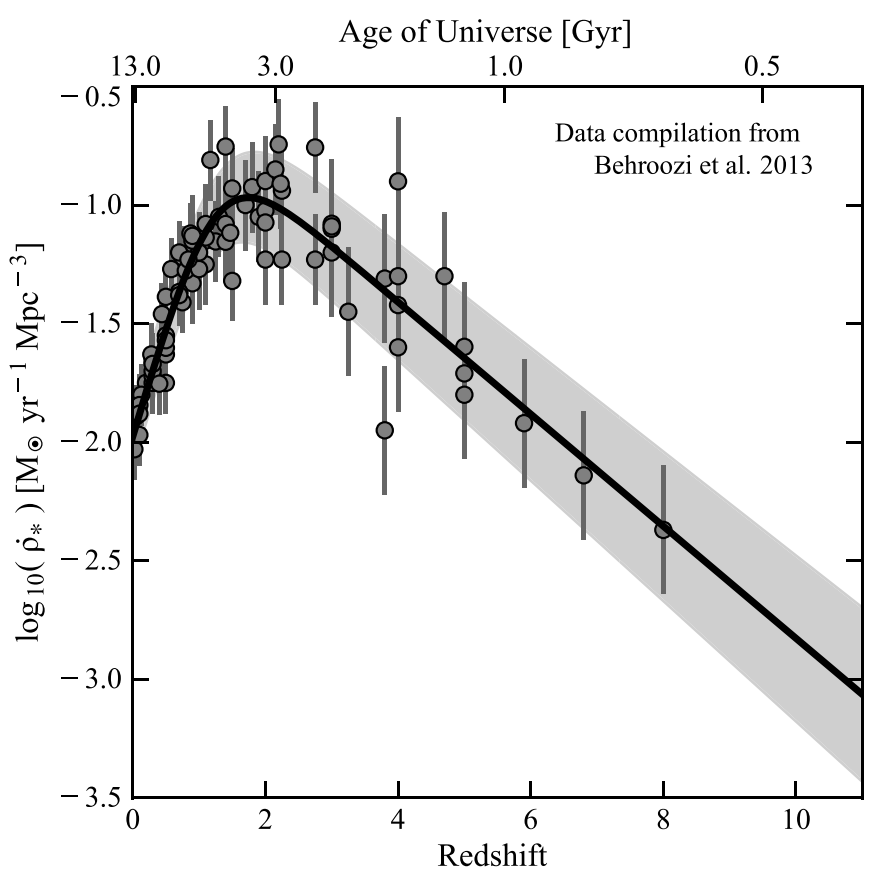

Figure 10. Cosmic star formation rate (CSFR) as a function of redshift. Points show the compilation of recent CSFR measurements from Behroozi et al. (2013), adopting from those authors the corrections for dust attenuation and more realistic systematic errors. The solid line shows the best-fit double power law model from Behroozi et al. (2013), and the shaded region demarcates the $1 \sigma$ systematic uncertainties.

Belczynski et al. 2005):

$$
\operatorname{SNR}(t)= \begin{cases}0 & \text { for } t<0.04 \mathrm{Gyr} \\ K \eta_{\mathrm{Ia}} \frac{f_{P}}{1-f_{P}} & \text { for } 0.04<\mathrm{t}<0.5 \mathrm{Gyr} \\ \eta_{\mathrm{Ia}} t^{-1} & \text { for } t>0.5 \mathrm{Gyr}\end{cases}
$$

Here $\eta_{\text {Ia }}$ indicates the efficiency of generating SN Ia progenitor systems, in units of SN Ia, $\mathrm{yr}^{-1} M_{\odot}^{-1}$, and $f_{P}$ sets the fraction of all SNe Ia that arise from the prompt channel. The constant $\mathrm{K}$ is defined by the time thresholds that delineate this model:

$$
K=\ln \left(t_{\max } / t_{1}\right) /\left(t_{1}-t_{\min }\right),
$$

where $t_{\min }=0.04 \mathrm{Gyr}$ as defined above, $t_{1}=0.5 \mathrm{Gyr}$ marks the abrupt transition from the constant rate to the power law, and $t_{\max }=13.3 \mathrm{Gyr}$ is the maximum age of a WD in the current universe-using our assumed $\Lambda C D M$ cosmology and assuming star formation began at $z=20$. For these values, we have $K=7.132$. With this simple DTD model, we can allow $\eta_{\text {Ia }}$ and $f_{P}$ to be free parameters, and fit to the data to find the observed efficiency and prompt Ia fraction.

To convert from this DTD model into a prediction for SN Ia rates, we convolve this DTD with a parameterized representation of the cosmic star formation history, giving us a prediction for the observable $\operatorname{SNR}(z)$. For this exercise we use the recent compilation of measurements of the cosmic star formation rate $(\operatorname{CSFR}(z))$ from Behroozi et al. (2013), shown in Figure 10. The precise shape of the CSFR curve at $z>2$ is still a matter of debate, but for our purposes here we take the Behroozi et al. curve and associated systematic uncertainties to be representative of the current state of the art (but see Graur et al. 2014, for further evaluation of star formation history variation). 
Table 6

Observational Constraints on the SN Ia Delay Time Distribution ${ }^{\mathrm{a}}$

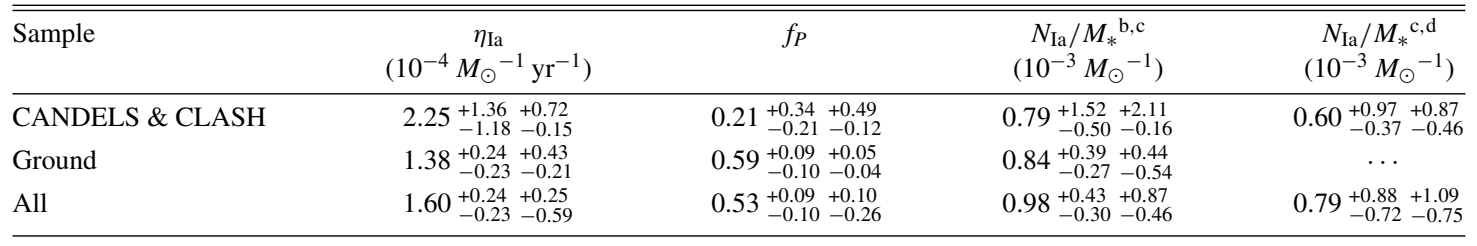

Notes.

${ }^{a}$ Errors give first statistical then systematic uncertainties.

b Assuming a $t^{-1}$ delay time model of the form given in Equation (4).

${ }^{c}$ Using the Behroozi et al. (2013) cosmic star formation history, which assumes a Chabrier (2003) IMF.

${ }^{\mathrm{d}}$ Using the SN Ia rate data directly, without any DTD model assumption.

The construction of our bifurcated DTD model is reminiscent of the two-component "A+B" model (Mannucci et al. 2005; Scannapieco \& Bildsten 2005), but it has closer ties to recent theoretical predictions from binary population synthesis models. For example, Ruiter et al. (2013) found that a "violent merger" DD model predicts a $t^{-1}$ power law shape for long delay SNe, but also includes a very prompt component that arises from a distinct subset of binary systems. A separate prompt channel for SN Ia explosions could also arise from a single degenerate pathway with a helium star donor (Wang et al. 2009; Claeys et al. 2014).

\subsection{DTD Model Fitting Results}

To find the most likely values for our two parameters $\eta_{\mathrm{Ia}}$ and $f_{P}$, we use three SN Ia rate data sets. First, we define the "All" data set, utilizing all available (non-redundant) volumetric rate measurements from the literature (see Graur et al. 2014, for a compilation table). Second, our "Ground" subsample picks out the 13 independent rate measurements from ground-based surveys. Finally, our "CANDELS+CLASH" sample isolates those two companion HST surveys.

The first three columns of Table 6 summarize the maximum likelihood values for our DTD parameters $\eta_{\text {Ia }}$ and $f_{P}$, when fitting to each of these subsamples. When using all of the available SN Ia survey data, we find $\eta_{\text {Ia }}=\left(1.60_{\text {stat0.236sys } 0.59}^{ \pm 0.24} \pm 0.25\right) \times 10^{-4} \mathrm{SN}$ Ia $\mathrm{yr}^{-1} M_{\odot}^{-1}$ and $f_{P}=0.53_{\text {stat } 0.10 \text { sys } 0.26}^{ \pm 0}$. This is a statistically acceptable fit, with a reduced $\chi^{2}$ of $0.9(p$-value $=0.67)$.

Fitting to the ground-based data alone, we find very similar best-fit parameters, with the prompt SN Ia fraction inching up to $f_{P}=0.59$ and the efficiency remaining at $\eta_{\text {Ia }} \sim 1.5$. When we isolate the HST CANDELS and CLASH surveys, we get much larger uncertainties, but perhaps also a subtle hint at tension between the ground- and HST-based results: from the CANDELS+CLASH sample we get $f_{P}=0.21_{\text {stat } 0.21 \text { sys0.12 }}^{ \pm 0.49}$. The difference in these best-fit parameters reflects a (very) mild disagreement between the ground-based, primarily low- $z$ rate measurements and the high- $z$ constraints from $H S T$.

The source of this deviation is easily seen in Figure 11, where we plot two $\operatorname{SNR}(z)$ curves derived from the bifurcated DTD model. The (magenta) solid line shows the best fit to the ground-based data alone, with $f_{P}=0.6$. The (green) dashed line sets the prompt fraction to $20 \%$, the best fit value for the CANDELS+CLASH data sample. These two HST surveys find a relatively low $\mathrm{SN}$ Ia rate at all redshifts $z \gtrsim 1$, which pulls the best-fit curve downward at high redshift, leading to the low best-fit $f_{P}$. We can also see the slight tension between ground and HST measures in Figure 12, where we show confidence regions in the $\eta_{\text {Ia }}$ vs. $f_{P}$ parameter space. The $68 \%$ contours from the ground- and HST-based surveys fall just short of overlapping. This discrepancy is only slightly above $1 \sigma$ in significance, and comes with all the caveats cited above regarding the method for combining data from disparate surveys. Nevertheless, these HST data do sample the redshift range with the greatest leverage for constraining $f_{P}$, so the scarcity of high- $z$ SN Ia detections in multiple $H S T$ surveys should not be discounted.

Table 6 and Figure 12 also present the total number of SNe Ia per stellar mass, $N_{\mathrm{Ia}} / M_{*}$. This is computed by integrating the $\mathrm{SN}$ Ia rate over a Hubble time, and dividing by the total mass of formed stars. For the denominator, we take the integral of the Behroozi et al. (2013) CSFR $(z)$ curve from Figure 10 (which assumes a Chabrier 2003 stellar initial mass function). To get the numerator-the total number of SN Ia explosions in a Hubble time-we can integrate the best-fit DTD-based SNR $(z)$ model for each subsample of rate measurements. Those values are reported in the fourth column of Table 6 . In the fifth column we list an alternative calculation, now directly integrating the $\operatorname{SNR}(z)$ data, without reference to any DTD model. The latter approach yields a much less precise constraint, but it is more appropriate for use as a test of progenitor models, because it does not presuppose any particular shape for the DTD. Note that we do not measure a data-only constraint from the ground-based subsample because it does not reach a high enough redshift.

Figure 12 shows a color map in the background, reflecting the variation of $N_{\text {Ia }} / M_{*}$ within the $\eta_{\text {Ia }}-f_{P}$ plane (assuming that the DTD follows our two-component toy model). The contours derived from both the ground-based and $H S T$ surveys are roughly aligned along lines of constant $N_{\text {Ia }} / M_{*}$ (a singlecolor ridge in the color map). Hence the tight model-dependent constraints on $N_{\text {Ia }} / M_{*}$ as reported in Column 4 of Table 6.

All of the above $N_{\mathrm{Ia}} / M_{*}$ measurements are consistent with a value of roughly $1 \times 10^{-3} M_{\odot}{ }^{-1}$. This is fully consistent with past measures of the volumetric rates, using similar stellar initial mass function (IMF) assumptions (e.g., Graur et al. 2011). Other observational constraints, such as cluster SN Ia rates, have recently found values closer to $2 \times 10^{-3} M_{\odot}{ }^{-1}$ (Maoz \& Badenes 2010)—still consistent within the large error bars. However, theoretical predictions from binary population synthesis models are frequently lower by factors of 10 or more (Bours et al. 2013). This discrepancy between theory and observation remains one of the key concerns in the SN Ia progenitor problem.

\subsection{Interpretation and Speculation}

As described above, our analysis of all available SN Ia rate measurements suggests that the fraction of SN Ia explosions occurring $<500 \mathrm{Myr}$ after formation is $f_{P} \sim 50 \%$. This 


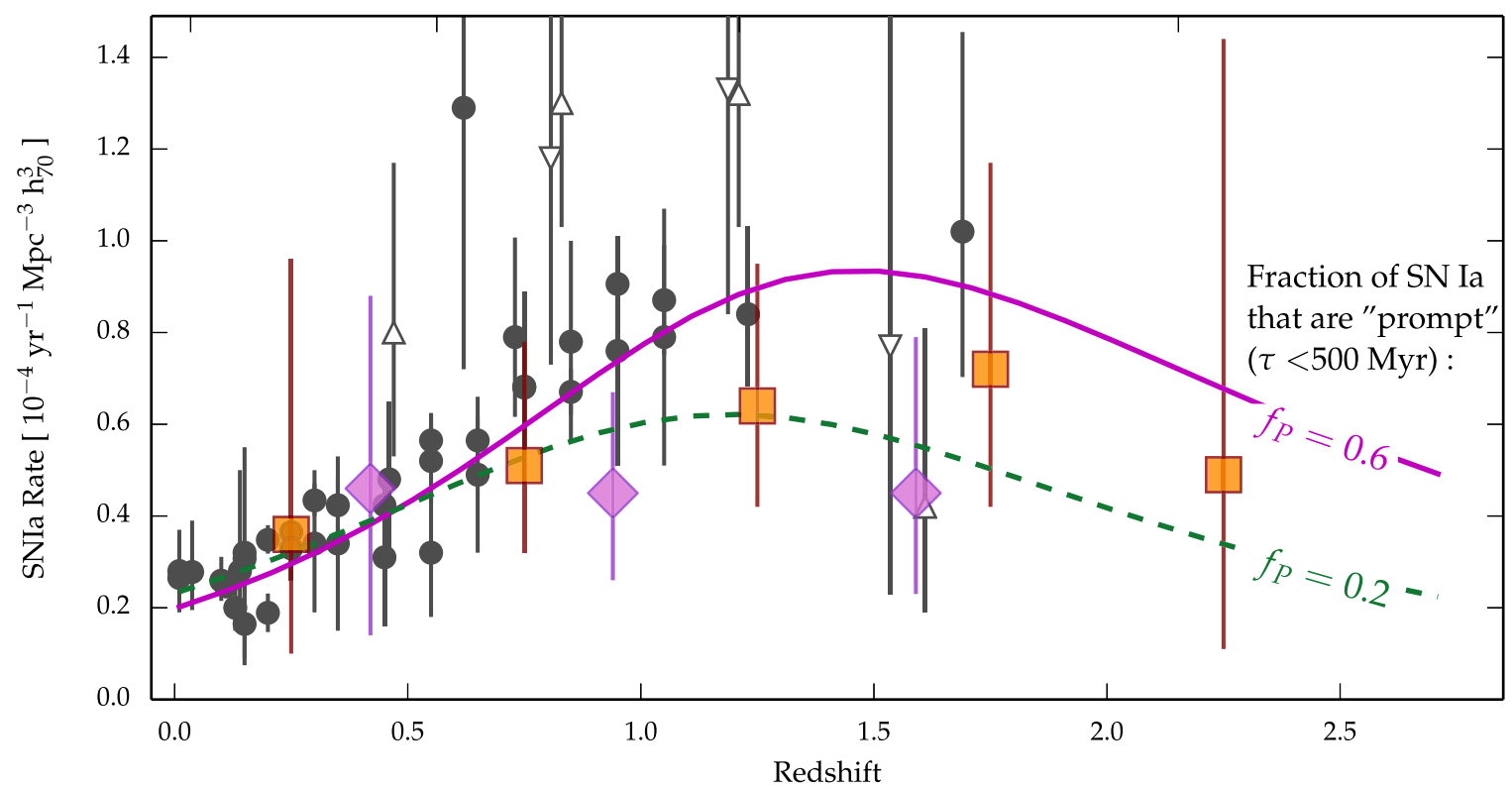

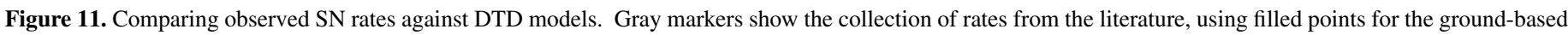

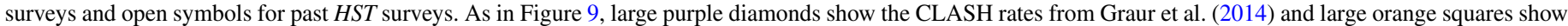

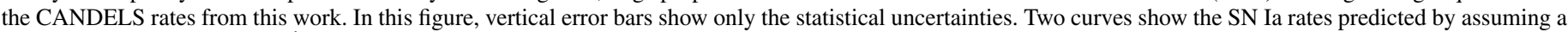

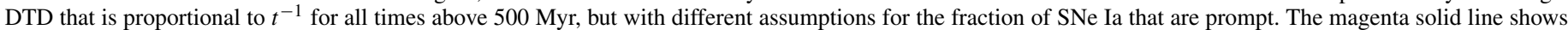

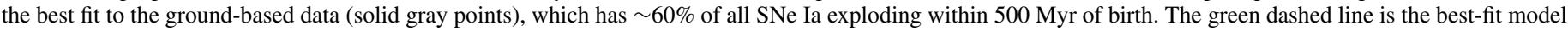
when using the CANDELS+CLASH data alone, for which the prompt SN Ia fraction is $20 \%$.

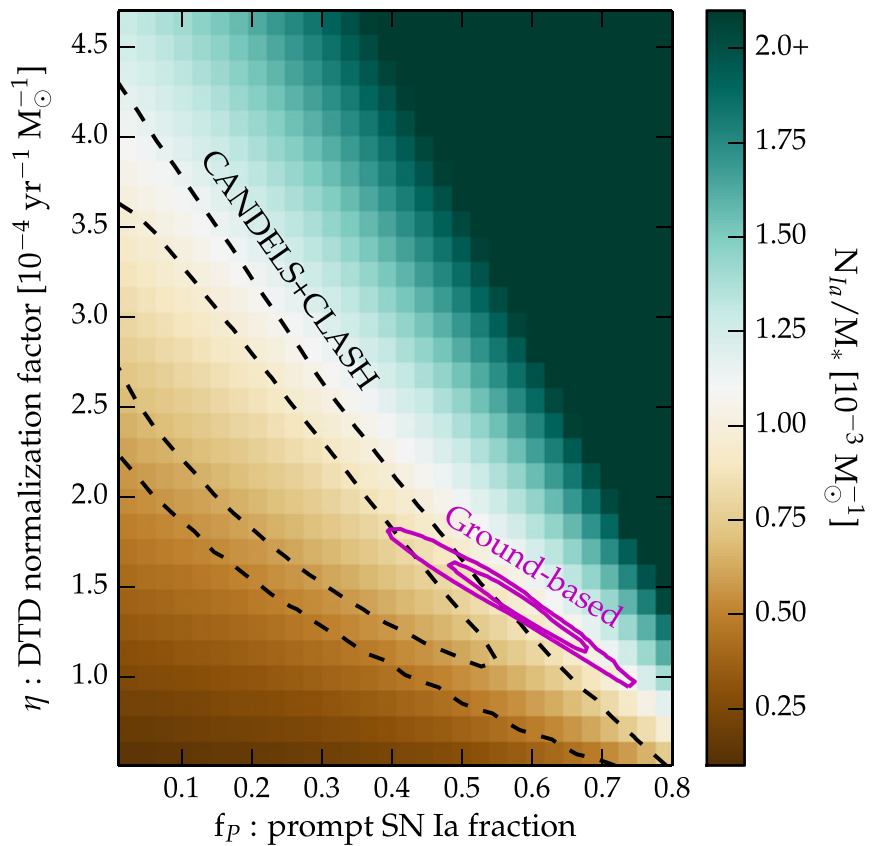

Figure 12. Constraints on the DTD normalization factor and the fraction of SNe Ia that are prompt explosions. Contours show the $68 \%$ and $95 \%$ confidence regions for the baseline assumptions (mid-dust, mid-rates) in the $\eta_{\text {Ia }}$ vs. $f_{P}$ parameter space. The background color map indicates the time-integrated SN Ia efficiency, $N_{\mathrm{Ia}} / M_{*}$, for each point in that parameter space. Dashed contours show the confidence regions derived from only HSTdata, reaching to $z \sim 2.5$. Solid contours are from the collection of all ground-based SN surveys, dominated by measurements at $z<1$.

observed value of $f_{P}$ is consistent with the simplistic assumption of $\mathrm{a} \mathrm{t}^{-1}$ DTD that continues without truncation all the way down to $40 \mathrm{Myr}$, which yields $f_{P}=0.43$. A prompt fraction close to $50 \%$ is also observationally supported by several lines of evidence in the local universe. Mannucci et al. (2006) first proposed that roughly half of all SN Ia explode promptly after formation, based on observations of SN Ia host galaxies at low redshift. Building on that work, Raskin et al. (2009) used measurements of low- $z$ SN Ia environments on sub-galactic scales to infer that most of those prompt SNe Ia explode at 200-500 Myr. Mennekens et al. (2013) used binary population synthesis (BPS) to predict the distribution of chemical enrichment in our galaxy over time. Comparing this to observations of $[\mathrm{Fe} / \mathrm{H}]$ in nearby $\mathrm{G}$ dwarfs, they infer that prompt explosions must make up a large fraction of the SN Ia population (and thereby contribute to rapid galactic enrichment).

Can this measurement of the prompt SN Ia fraction be used to distinguish SD and DD progenitor models? BPS calculations generally agree that SD pathways preferentially generate prompt SN Ia explosions. In particular, SD progenitor models in which the companion is a naked He star are found to peak at $t \sim 100 \mathrm{Myr}$ after formation, while those with a normal main sequence or giant companion preferentially explode at 200-500 Myr (Wang et al. 2009; Mennekens et al. 2010; Greggio 2010; Claeys et al. 2014). Some BPS modeling also finds that DD progenitors could contribute substantially to the population of SN Ia explosions younger than 500 Myr (Ruiter et al. 2009; Greggio 2010; Ruiter et al. 2013), although recent work by Claeys et al. (2014) suggests that the DD pathway does not dominate the DTD until $t>500$ Myr. At the moment, we can only say that a prompt fraction $f_{P} \sim 50 \%$ is commonly predicted by models that include both SD and DD progenitors-but it may be possible in a pure DD model as well.

When we analyze the HST sample in isolation, we find a hint that $f_{P}$ could be closer to $\sim 20 \%$, though further analysis and a larger SN Ia sample will be needed to improve this measurement. Let us indulge in a bit of speculation and suppose that these future improvements confirm that $f_{P}$ is close to $20 \%$ at high 
redshifts. We would then need a theoretical explanation for how the prompt component of the SN Ia population could be suppressed at redshifts $z>1.5$. One possible explanation would be that the prompt SN Ia component is dominated by an SD progenitor pathway with a strong metallicity dependence. Such a metallicity dependence has been proposed by requiring an optically thick wind from the WD to regulate the mass transfer rate, allowing the WD to grow to the Chandrasekhar mass limit (Hachisu et al. 1996, 1999). This wind would be absent in low metallicity WDs, resulting in a suppression of prompt SN Ia explosions in the early universe (Kobayashi et al. 1998; Kobayashi \& Nomoto 2009). No such metallicity threshold is expected for DD models, so this scenario could provide a clean way to disentangle the relative contributions of those two progenitor pathways.

\section{SUMMARY}

We have presented a sample of $65 \mathrm{SNe}$ from the five CANDELS fields. This sample, collected in concert with the CLASH SN search, is the first to extend SN Ia detections beyond $z=2$, and the first to detect $\mathrm{SNe}$ at $z \sim 1.5$ in rest-frame optical bands. These SNe have been classified primarily through the application of STARDUST, a new Bayesian photometric classifier that is optimized for working with light curves of high redshift SNe. We have spectroscopic redshifts from the SN and/ or host galaxy for $82 \%$ of the sample (53 of 65), although we rely on photometric redshifts for $43 \%$ of the $\mathrm{SNe}$ at $z>1.5$ (6 of 14). Our SN classification probabilities are in general tightly constrained by well-sampled light curves, rest-frame UV-optical colors, and well-defined redshifts. The primary sources for potential systematic biases in our classifications are (1) a redshift-dependent prior describing the relative fraction of SNe that are Type Ia, and (2) the assumed distribution of dust extinction values. For the former, a test using host galaxy information to replace the class prior indicates that our systematic uncertainty estimates are appropriate.

From the CANDELS SN sample we have measured the volumetric $\mathrm{SN}$ Ia rate in five redshift bins reaching to $z=$ 2.5. We find that the CANDELS SN Ia rate measurement at $z \sim 1.25$ is a factor of 2 lower ( $\sim 2$ sigma) than past HST rate measurements at the same redshift (Dahlen et al. 2008; Barbary et al. 2012), but is consistent with the concurrently measured SN Ia rates from CLASH (Graur et al. 2014). We attribute this discrepancy to Poisson noise, due to the very small sample sizes in all of these HST surveys. At higher redshifts the CANDELS rate measurements remain approximately flat with redshift.

Combining these CANDELS results with other surveys from $H S T$ and from the ground, we have examined the constraints that can be placed on SN Ia DTD models (Table 6, Figure 11). We have invoked a simple two-component model with a $t^{-1}$ distribution for long delay times $(t>500 \mathrm{Myr})$, and a constant rate at shorter times. We find that the ground-based rates (primarily at $z<1$ ) and the full sample of all available SN Ia rates are both best matched with a prompt SN Ia fraction of $f_{P}=0.5$. When the CANDELS+CLASH surveys are analyzed in isolation, the best fit for the prompt SN Ia fraction falls to $f_{P}=0.21_{-0.21-0.12}^{+0.34+0.49}$. This is much lower, but with very large error bars.

Collectively, the constraints from all available volumetric SN data indicate that the prompt SN Ia fraction cannot be much larger than $60 \%$. As described above, there is room for substantial improvement in our measurement of the prompt SN Ia fraction - without needing to acquire more data. System- atic uncertainties can be reduced (or at least better understood) by combining the existing HST surveys in a composite analysis that handles SN classification and dust obscuration in a consistent manner. Increasing the high- $z$ SN Ia sample size would also help, of course. The HST Frontier Fields initiative will provide the next opportunity for new high- $z$ SN discoveries. This program will utilize $\sim 900$ orbits of HST observations for very deep imaging of six massive galaxy clusters over three years. An approved HST program for SN discovery and follow-up (PI: Rodney, PID: 13386) is expected to deliver an SN Ia sample that reaches out to $z \sim 3$.

This work is dedicated to the memory of our dear friend and colleague, Tomas Dahlen.

The authors would like to thank the referee, Massimo Della Valle, for helpful comments and suggestions. We also thank the STScI science support staff for their extraordinary efforts in executing the MCT programs, especially program coordinators Patricia Royle and Beth Perriello, as well as the entire STScI scheduling team that made our HST ToO program possible. Thanks also to Ori Fox, Patrick Kelly, Isaac Shivvers, Brad Tucker, and WeiKang Zheng for assistance with Keck observations; to Ismael Botti, Alice Mortlock, and Omar Almaini for redshift and AGN classifications of SN candidates in the UDS field; to Stephane Blondin for VLT spectral reductions and assistance with SNID modifications; to Jennifer Lotz and Ryan Foley for discussions improving the measurement and application of SN host galaxy morphologies; and to Thomas Holoien and Mark Ziegler for "fake SN" searching assistance.

The CANDELS program was supported by NASA through grant HST-GO-12060, and the SN-MCT program through grant HST-GO-12099 from the Space Telescope Science Institute, which is operated by Associated Universities for Research in Astronomy, Inc., under NASA contract NAS 5-26555. Support for S.A.R. was provided by NASA through Hubble Fellowship grant HST-HF-51312. Support for this research at Rutgers University was provided in part by NSF CAREER award AST0847157 to S.W.J. The Dark Cosmology Centre is supported by the Danish National Research Foundation. R.P.K. thanks the National Science Foundation for AST-1211196, and the John Simon Guggenheim Foundation for support. This work was supported by NASA Keck PI Data Awards (to Rutgers University, PI: S.W.J.), administered by the NASA Exoplanet Science Institute. J.M.S. is supported by an NSF Astronomy and Astrophysics Postdoctoral Fellowship under award AST1302771. A.V.F. is also grateful for the support of the National Science Foundation (NSF) grant AST-1211916, the TABASGO Foundation, and the Christopher R. Redlich Fund.

This research was based primarily on observations made with the NASA/ESA Hubble Space Telescope, delivered by the data archive team at the Space Telescope Science Institute (STScI), which is operated by the association of Universities for Research in Astronomy, Inc. under NASA contract NAS 5-26555. We used the HST Science Archive hosted by the Canadian Astronomy Data Centre (CADC/NRC/CSA), as well as the Mikulski Archive for Space Telescopes (MAST).

This work is based in part on observations made with ESO telescopes at the La Silla Paranal Observatory under program IDs 086.A-0660 and 088.A-0708.

Some of the data presented herein were obtained at the W. M. Keck Observatory from telescope time allocated to NASA through the agency's scientific partnership with the California Institute of Technology and the University of California. The 
Table 7

Host Galaxy Likelihood Distributions for Defining Host-based Classification Priors ${ }^{\mathrm{a}}$

\begin{tabular}{lcc}
\hline \hline Category & $P(D \mid \mathrm{Ia})$ & $P(D \mid \mathrm{CC})$ \\
\hline & Morphology & \\
\hline spheroid (E,S0) & $0.25 \pm 0.03$ & $0.01 \pm 0.01$ \\
spheroid+disk (S0,Sa) & $0.26 \pm 0.03$ & $0.15 \pm 0.02$ \\
disk (Sb,Sbc,Sc) & $0.35 \pm 0.03$ & $0.53 \pm 0.03$ \\
disk+irregular (Sc,Scd) & $0.10 \pm 0.02$ & $0.20 \pm 0.02$ \\
irregular (Scd,Irr) & $0.04 \pm 0.01$ & $0.11 \pm 0.02$ \\
\hline & $0.35 \pm 0.06$ & \\
\hline Passive $(B-K>3.75)$ & $0.52 \pm 0.08$ & $0.08 \pm 0.06$ \\
Active $(2.75<B-K<3.75)$ & $0.13 \pm 0.04$ & $0.30 \pm 0.08$ \\
Starburst $(B-K<2.75)$ & SED Type ${ }^{\mathrm{b}}$ & \\
\hline
\end{tabular}

Notes.

a Likelihood estimates follow the galsnid for malism (Foley \& Mandel 2013), using LOSS host galaxy data compiled in Leaman et al. (2011).

b Approximate rest-frame $B-K$ colors are given in Vega mags. In AB mags the thresholds are 0.82 and 1.82 .

Observatory was made possible by the generous financial support of the W. M. Keck Foundation. The authors wish to recognize and acknowledge the very significant cultural role and reverence that the summit of Mauna Kea has always had within the indigenous Hawaiian community. We are most fortunate to have the opportunity to conduct observations from this mountain.

This work is based in part on observations obtained at the Gemini Observatory, which is operated by the Association of Universities for Research in Astronomy, Inc., under a cooperative agreement with the NSF on behalf of the Gemini partnership: the National Science Foundation (United States), the National Research Council (Canada), CONICYT (Chile), the Australian Research Council (Australia), Ministério da Ciência, Tecnologia e Inovação (Brazil) and Ministerio de Ciencia, Tecnología e Innovación Productiva (Argentina). Contributing data came from Gemini programs GN-2011A-Q-14, GN-2011B-Q18, GN-2012A-Q-32, GN-2013A-Q-25, GS-2011A-Q-16, GS2011B-Q-18, GS-2012A-Q-17, and GS-2013A-Q-19.

This research has made use of the VO Datascope, developed with the support of the National Science Foundation under Cooperative Agreement AST0122449 with the Johns Hopkins University, and hosted by the Astrophysics Science Division and the High Energy Astrophysics Science Archive Research Center (HEASARC), a service of Goddard Space Flight Center and the Smithsonian Astrophysical Observatory. This research has made use of the SIMBAD database, operated at CDS, Strasbourg, France. This research has made use of NASA's Astrophysics Data System Bibliographic Services. This research made use of Astropy, a community-developed core Python package for Astronomy (Astropy Collaboration et al. 2013).

Facilities: HST (WFC3), Keck (DEIMOS), Keck (LRIS), VLT (X-shooter), VLT (FORS2), Gemini (GMOS-N), Gemini (GMOS-S)

\section{APPENDIX A}

\section{THE HOST-BASED CLASS PRIOR}

In this Appendix we perform a test to see if our systematic uncertainty estimates are accurately reflecting the bias that could arise from using an incorrect redshift-dependent prior $P(\mathrm{Ia}, z)$ in the STARDUST classifier. For this test we measure the change in
Table 8

Change in Observed SN Ia Counts and the Volumetric Rate When Adopting the Host Galaxy Prior

\begin{tabular}{lccc}
\hline \hline Redshift & $\Delta N_{\text {obs }}$ & $\Delta$ SNR & \% of Sys. Err. \\
\hline 0.25 & +0.13 & +0.03 & 26 \\
0.75 & +1.99 & +0.14 & 62 \\
1.25 & +0.93 & +0.07 & 21 \\
1.75 & +0.41 & +0.05 & 10 \\
2.25 & +0.33 & +0.13 & 28 \\
\hline
\end{tabular}

the volumetric SN Ia rate that occurs when we adopt a redshiftindependent prior based on host galaxy data.

It is well established that CCSNe are almost never observed in galaxies dominated by older stellar populations (passive, red ellipticals). The rate of SNe Ia per unit mass increases in galaxies with young stellar populations, but not as sharply as the specific CCSN rate. The galsnid SN classifier (Foley \& Mandel 2013) exploits these relationships between SNe and their host environments to define a posterior classification probability that is completely independent of the SN photometry.

The galsnid approach relies on a database of observed SN host galaxy properties (morphology, color, luminosity, etc.) from the Lick Observatory Supernova Survey (LOSS; Leaman et al. 2011). These data are used to define a set of likelihoods $P\left(\mathbf{D}_{h} \mid\right.$ Type $)$ giving the probability of observing a set of host galaxy properties, $\mathbf{D}_{h}$, if the $\mathrm{SN}$ hosted in that galaxy is of the given type. The galsnid posterior classification probability for Type Ia is

$$
P\left(\mathrm{Ia} \mid \mathbf{D}_{h}\right)=k_{h}^{-1} P(\mathrm{Ia}) \prod_{i=1}^{n} P\left(D_{i} \mid \mathrm{Ia}\right),
$$

where $P(\mathrm{Ia})$ is the Ia classification prior, $D_{h, i}$ are the $n$ observed host galaxy properties, and $k_{h}$ is a normalization term, which ensures that the sum of posterior classification probabilities for all SN Ia and CCSN types is unity. For our implementation of galsnid, the prior $P$ (Ia) — and the corresponding priors for $\mathrm{CCSNe}$ - are fixed to match the observed fraction of $\mathrm{SNe}$ in a volume-limited sample that are of each type: $P(\mathrm{Ia})=0.25$, $P(\mathrm{Ib} / \mathrm{c})=0.19$, and $P(\mathrm{II})=0.57$ (Smartt et al. 2009; Li et al. 2011). For the host galaxy observables, $\mathbf{D}_{h}$, we employ the two quantities that provide the strongest discriminatory power according to Foley \& Mandel (2013): morphology and color.

Table 7 translates from the CANDELS categories for host galaxy morphology (spheroid, disk, or irregular) into their approximate counterparts on the Hubble sequence (E, S0, etc.). That table also describes the translation from the CANDELS SED types (passive, active, or starburst) into the rest-frame $(B-K)$ color.

For SNe in faint hosts where the host galaxy morphology is undefined, the galsnid posterior reflects only the constraint from color/SED type. In the two cases where the SN host galaxy is totally undetected, the photometric redshifts are unconstrained and galsnid simply reflects back the input prior, $P$ (Ia) in Equation (A1). The likelihood values in Columns 2 and 3 of Table 7 mimic Table 1 of Foley \& Mandel (2013) and provide all the information needed to determine $P$ (Ia, host) for any CANDELS SN host galaxy.

\section{A.1. Combining galsnid and STARDUST}

To incorporate the galsnid information into STARDUST, we adopt the galsnid posterior, $P\left(\mathrm{Ia} \mid \mathbf{D}_{\mathbf{h}}\right)$, as a redshift-independent 
Table 9

51 Supernovae with $z<1.5$

\begin{tabular}{|c|c|c|c|c|c|c|c|}
\hline Name & $\begin{array}{c}\text { R.A. } \\
(\mathrm{J} 2000)\end{array}$ & $\begin{array}{c}\text { Decl. } \\
(\mathrm{J} 2000)\end{array}$ & $P\left(\mathrm{Ia} \mid D_{z}\right)^{\mathrm{a}}$ & $P\left(\text { Ia } \mid D_{\text {host }}\right)^{\mathrm{b}}$ & $z_{\mathrm{SN}}{ }^{\mathrm{c}}$ & $( \pm)$ & $z$ Source $^{\mathrm{d}}$ \\
\hline GSD10Tum & $03: 32: 17.705$ & $-27: 50: 57.50$ & $0.00_{-0.00}^{+0.00}$ & $0.00_{-0.00}^{+0.00}$ & 0.124 & $(0.001)$ & host spec- $z$ \\
\hline COS12Cli & 10:00:15.958 & $+02: 12: 36.27$ & $0.29_{-0.28}^{+0.14}$ & $0.29_{-0.29}^{+0.03}$ & 0.187 & $(0.001)$ & host spec- $z$ \\
\hline GSD12Roc & 03:32:06.368 & $-27: 47: 26.63$ & $0.00_{-0.00}^{+0.00}$ & $0.00_{-0.00}^{+0.00}$ & 0.346 & $(0.003)$ & host spec- $z$ \\
\hline EGS11Tho & $14: 19: 31.775$ & $+52: 51: 56.16$ & $0.99_{-0.98}^{+0.01}$ & $0.99 \stackrel{+0.00}{-0.98}$ & 0.354 & $(0.001)$ & host spec- $z$ \\
\hline COS12Ken & $10: 00: 36.010$ & $+02: 15: 26.53$ & $0.00_{-0.00}^{+0.00}$ & $0.00_{-0.00}^{+0.00}$ & 0.373 & $(0.001)$ & host spec- $z$ \\
\hline GND13She & $12: 36: 09.877$ & $+62: 14: 06.00$ & $0.18_{-0.18}^{+0.33}$ & $0.31_{-0.31}^{+0.25}$ & 0.473 & $(0.001)$ & host spec- $z$ \\
\hline GSD11Tay & $03: 32: 54.505$ & $-27: 47: 04.23$ & $0.67_{-0.64}^{+0.21}$ & $0.72_{-0.64}^{+0.12}$ & 0.535 & $(0.001)$ & host spec- $z$ \\
\hline GSD11For & $03: 32: 14.300$ & $-27: 47: 13.30$ & $0.41_{-0.41}^{+0.26}$ & $0.43_{-0.42}^{+0.13}$ & 0.578 & $(0.001)$ & host spec- $z$ \\
\hline GND13Bid & $12: 36: 41.325$ & $+62: 11: 42.21$ & $0.00_{-0.00}^{+0.00}$ & $0.00_{-0.00}^{+0.00}$ & 0.585 & $(0.001)$ & host spec- $z$ \\
\hline COS12Eis & 10:00:47.233 & $+02: 11: 50.50$ & $0.53_{-0.18}^{+0.14}$ & $0.699_{-0.01}^{+0.04}$ & 0.605 & $(0.001)$ & host spec- $z$ \\
\hline UDS11Gar & $02: 17: 39.633$ & $-05: 11: 37.14$ & $0.03_{-0.03}^{+0.09}$ & $0.08_{-0.08}^{+0.10}$ & 0.651 & $(0.001)$ & host spec- $z$ \\
\hline GSD11Wor & $03: 32: 10.739$ & $-27: 48: 07.17$ & $1.00_{-0.00}^{+0.00}$ & $1.00_{-0.00}^{+0.00}$ & 0.654 & $(0.001)$ & host spec- $z$ \\
\hline GSD11Roo & $03: 32: 31.587$ & $-27: 46: 12.53$ & $0.00_{-0.00}^{+0.00}$ & $0.01_{-0.00}^{+0.00}$ & 0.655 & $(0.001)$ & host spec- $z$ \\
\hline GSW11Jac & $03: 32: 32.300$ & $-27: 54: 20.55$ & $0.17_{-0.11}^{+0.13}$ & $0.18_{-0.06}^{+0.04}$ & 0.659 & $(0.001)$ & host spec- $z$ \\
\hline COS12Tru & 10:00:38.322 & $+02: 11: 36.41$ & $0.46_{-0.46}^{+0.32}$ & $0.52_{-0.52}^{+0.22}$ & 0.665 & $(0.001)$ & host spec- $z$ \\
\hline COS12Rea & 10:00:31.917 & $+02: 14: 16.15$ & $0.00_{-0.00}^{+0.00}$ & $0.00_{-0.00}^{+0.00}$ & 0.679 & $(0.001)$ & host spec- $z$ \\
\hline GSD11Buc & $03: 32: 28.800$ & $-27: 52: 32.00$ & $0.01_{-0.01}^{+0.01}$ & $0.10_{-0.10}^{+0.06}$ & 0.679 & $(0.001)$ & host spec- $z$ \\
\hline GSD11Har & $03: 32: 30.441$ & $-27: 45: 18.70$ & $0.32_{-0.32}^{+0.28}$ & $0.25_{-0.25}^{+0.12}$ & 0.681 & $(0.001)$ & host spec- $z$ \\
\hline COS12Aid & 10:00:15.252 & $+02: 17: 32.12$ & $0.00_{-0.00}^{+0.00}$ & $0.00_{-0.00}^{+0.00}$ & 0.731 & $(0.001)$ & host spec- $z$ \\
\hline GSD11Lin & $03: 32: 29.812$ & $-27: 49: 19.71$ & $0.00_{-0.00}^{+0.00}$ & $0.01_{-0.01}^{+0.00}$ & 0.734 & $(0.001)$ & host spec- $z$ \\
\hline GSD11Ada & 03:32:19.805 & $-27: 54: 10.04$ & $0.01_{-0.01}^{+0.02}$ & $0.01_{-0.01}^{+0.01}$ & 0.735 & $(0.001)$ & host spec- $z$ \\
\hline COS12Mon & 10:00:26.737 & $+02: 15: 13.74$ & $0.02_{-0.02}^{+0.06}$ & $0.49_{-0.49}^{+0.19}$ & 0.777 & $(0.001)$ & host spec- $z$ \\
\hline GND13Ful & $12: 36: 19.201$ & $+62: 15: 12.58$ & $0.00_{-0.00}^{+0.00}$ & $0.00+0.00$ & 0.783 & $(0.001)$ & host spec- $z$ \\
\hline GND12Bur & $12: 36: 32.536$ & $+62: 15: 32.62$ & $0.00_{-0.00}^{+0.00}$ & $0.00_{-0.00}^{+0.00}$ & 0.82 & $(0.13)$ & host+SN phot-z \\
\hline GND12Daw & $12: 36: 41.340$ & $+62: 18: 52.50$ & $0.00_{-0.00}^{+0.00}$ & $0.00_{-0.00}^{+0.00}$ & 0.83 & $(0.02)$ & SN spec- $z+$ phot $-z$ \\
\hline GND13Vin & $12: 37: 06.354$ & $+62: 15: 17.79$ & $0.11_{-0.11}^{+0.18}$ & $0.13_{-0.13}^{+0.09}$ & 0.840 & $(0.001)$ & host spec- $z$ \\
\hline GNW12Ger & $12: 37: 17.023$ & $+62: 20: 38.67$ & $0.05_{-0.03}^{+0.02}$ & $0.66_{-0.10}^{+0.00}$ & 0.850 & $(0.001)$ & host spec- $z$ \\
\hline GND13Roy & $12: 36: 46.223$ & $+62: 15: 27.13$ & $0.00_{-0.00}^{+0.00}$ & $0.00+0.00$ & 0.851 & $(0.001)$ & host spec- $z$ \\
\hline GSD11Van & $03: 32: 19.037$ & $-27: 47: 17.90$ & $0.05_{-0.05}^{+0.12}$ & $0.19_{-0.19}^{+0.18}$ & 0.886 & $(0.097)$ & host+SN phot-z \\
\hline EGS11Per & $14: 18: 28.318$ & $+52: 42: 45.88$ & $0.99_{-0.01}^{+0.00}$ & $1.00_{-0.00}^{+0.00}$ & 0.915 & $(0.001)$ & host spec- $z$ \\
\hline COS12Mik & 10:00:31.687 & $+02: 26: 13.73$ & $0.00_{-0.00}^{+0.00}$ & $0.01_{-0.00}^{+0.00}$ & 0.927 & $(0.001)$ & host spec- $z$ \\
\hline GND12Cal & $12: 37: 10.487$ & $+62: 15: 47.67$ & $1.00_{-0.00}^{+0.00}$ & $1.00_{-0.00}^{+0.00}$ & 0.941 & $(0.005)$ & host spec- $z$ \\
\hline GND13Wol & $12: 36: 58.946$ & $+62: 18: 10.15$ & $0.31_{-0.12}^{+0.08}$ & $0.58_{-0.05}^{+0.02}$ & 0.943 & $(0.001)$ & host spec- $z$ \\
\hline GSD11Mad & $03: 32: 18.781$ & $-27: 52: 42.05$ & $1.00+0.00$ & $1.00_{-0.00}^{+0.00}$ & 0.988 & $(0.001)$ & host spec- $z$ \\
\hline GNW12Gor & $12: 36: 20.704$ & $+62: 08: 45.08$ & $0.09_{-0.08}^{+0.10}$ & $0.23_{-0.15}^{+0.07}$ & 1.016 & $(0.001)$ & host spec- $z$ \\
\hline GND13Reh & $12: 36: 54.452$ & $+62: 11: 52.47$ & $0.00_{-0.00}^{+0.00}$ & $0.09 \stackrel{+0.03}{-0.06}$ & 1.019 & $(0.001)$ & host spec- $z$ \\
\hline GND13Jay & $12: 36: 41.380$ & $+62: 11: 30.02$ & $1.00_{-0.01}^{+0.00}$ & $1.00_{-0.00}^{+0.00}$ & 1.03 & $(0.01)$ & host spec- $z$ \\
\hline GND13Gar & $12: 36: 40.806$ & $+62: 11: 14.16$ & $1.00_{-0.00}^{+0.00}$ & $1.00_{-0.00}^{+0.00}$ & 1.067 & $(0.008)$ & SN spec- $z+$ phot $-z$ \\
\hline COS12Her & 10:00:47.446 & $+02: 15: 17.74$ & $0.01_{-0.00}^{+0.00}$ & $0.02_{-0.01}^{+0.03}$ & 1.082 & $(0.077)$ & host+SN phot- $z$ \\
\hline GSD12Agn & $03: 32: 25.902$ & $-27: 50: 19.62$ & $0.80_{-0.71}^{+0.13}$ & $0.82_{-0.63}^{+0.07}$ & 1.095 & $(0.001)$ & host spec- $z$ \\
\hline EGS13Tan & $14: 20: 02.098$ & $+53: 00: 16.36$ & $0.15_{-0.08}^{+0.05}$ & $0.35_{-0.07}^{+0.07}$ & 1.137 & $(0.439)$ & host+SN phot- $z$ \\
\hline GND12Tom & $12: 36: 42.543$ & $+62: 18: 21.93$ & $0.86_{-0.75}^{+0.09}$ & $0.85_{-0.63}^{+0.06}$ & 1.14 & $(0.001)$ & host spec- $z$ \\
\hline EGS13Wai & $14: 20: 28.534$ & $+53: 04: 58.61$ & $1.00_{-0.00}^{+0.00}$ & $1.00_{-0.00}^{+0.00}$ & 1.166 & $(0.001)$ & host spec- $z$ \\
\hline GND13Cam & $12: 37: 07.354$ & $+62: 10: 26.90$ & $1.00_{-0.01}^{+0.00}$ & $1.00_{-0.00}^{+0.00}$ & 1.222 & $(0.002)$ & host spec- $z$ \\
\hline GSD11Was & $03: 32: 20.856$ & $-27: 49: 41.48$ & $1.00_{-0.00}^{+0.00}$ & $1.00_{-0.00}^{+0.00}$ & 1.33 & $(0.02)$ & SN spec- $z+$ phot $-z$ \\
\hline GND12Whe & $12: 36: 24.220$ & $+62: 17: 39.70$ & $0.00_{-0.00}^{+0.00}$ & $0.00_{-0.00}^{+0.00}$ & 1.335 & $(0.005)$ & host spec- $z$ \\
\hline EGS13Cha & $14: 20: 24.200$ & $+53: 03: 37.50$ & $0.36_{-0.14}^{+0.01}$ & $0.80_{-0.11}^{+0.01}$ & 1.40 & $(0.01)$ & host spec- $z$ \\
\hline EGS11Oba & $14: 20: 32.663$ & $+53: 02: 48.18$ & $0.91_{-0.17}^{+0.02}$ & $0.90_{-0.05}^{+0.00}$ & 1.409 & $(0.001)$ & host spec- $z$ \\
\hline EGS11Nix & $14: 20: 48.603$ & $+53: 00: 26.47$ & $0.40_{-0.11}^{+0.01}$ & $0.58_{-0.10}^{+0.04}$ & 1.451 & $(0.077)$ & host+SN phot-z \\
\hline GND12Kin & $12: 37: 13.005$ & $+62: 16: 30.83$ & $0.00_{-0.00}^{+0.00}$ & $0.00_{-0.00}^{+0.00}$ & 1.46 & $(0.07)$ & host+SN phot-z \\
\hline GND13Fin & $12: 37: 18.173$ & $+62: 17: 09.65$ & $0.03_{-0.03}^{+0.06}$ & $0.02_{-0.01}^{+0.01}$ & 1.488 & $(0.001)$ & host spec- $z$ \\
\hline
\end{tabular}

Notes.

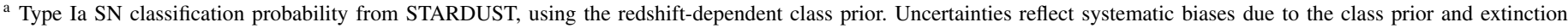
assumptions (Sections 4.2 and 4.3).

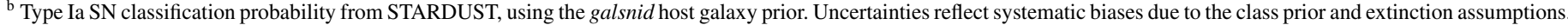

${ }^{\mathrm{c}}$ Posterior redshift and uncertainty, as determined by the STARDUST light curve fit.

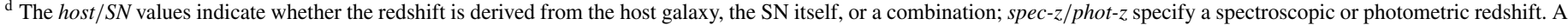
value of host $+S N$ phot $-z$ means the redshift is derived from a STARDUST light curve fit, with the host galaxy phot- $z$ used as a prior. 


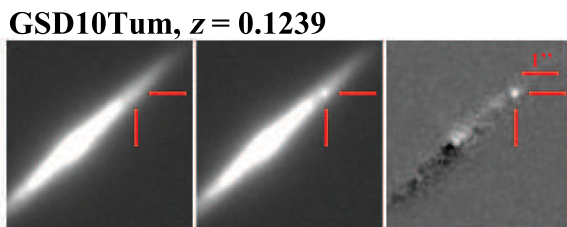

EGS11Tho, $z=0.354$

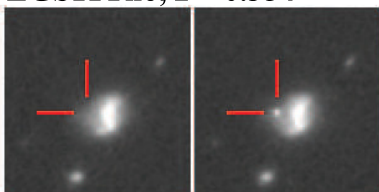

GSD11Tay, $z=0.5351$

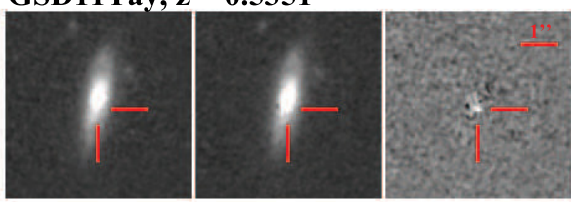

COS12Eis, $z=0.605$

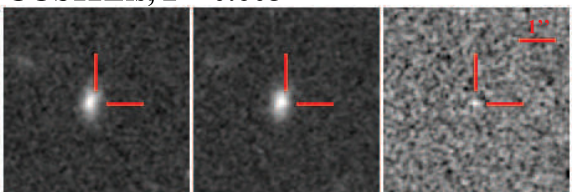

GSD11Roo, $z=0.655$

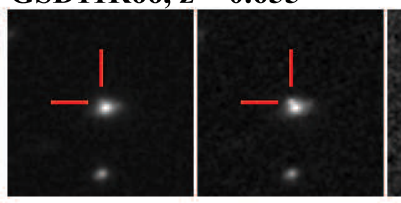

COS12Rea, $z=0.679$

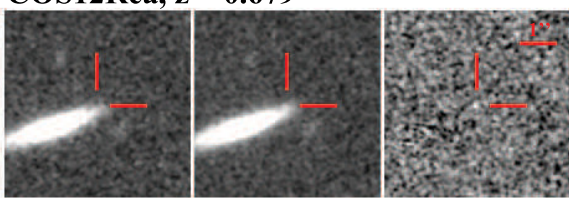

COS12Aid, $z=0.731$

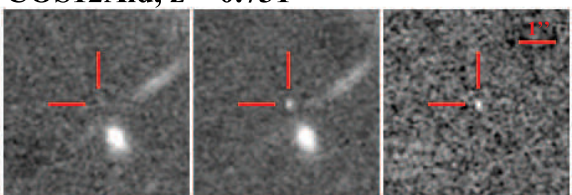

COS12Mon, $z=0.777$

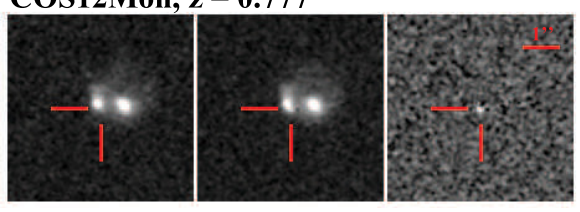

GND12Daw, $z=0.83 \pm 0.02$

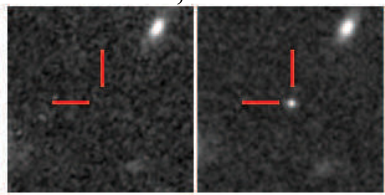

COS12Cli, $z=0.187$

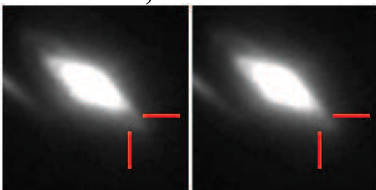

COS12Ken, $z=0.371$

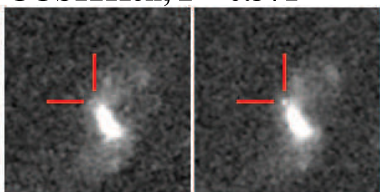

GSD11For, $z=0.578$

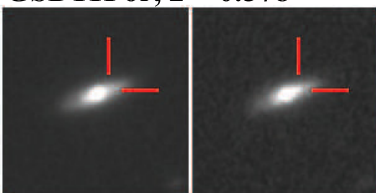

UDS11Gar, $z=0.651$

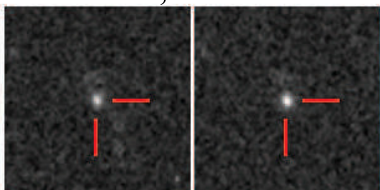

GSW11Jac, $z=0.659$

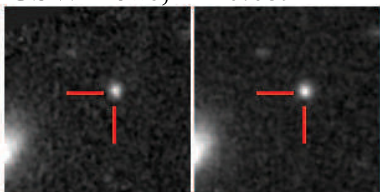

GSD11Buc, $z=0.6787$

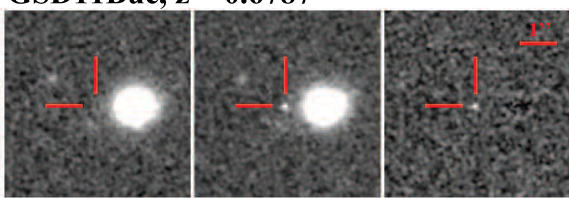

GSD11Lin, $z=0.81 \pm 0.15$

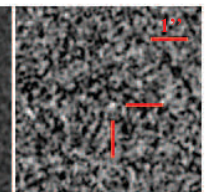

GND13Ful, $z=0.783$

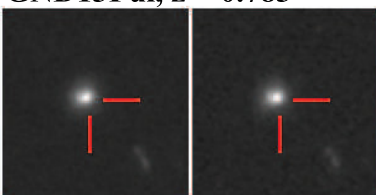

GND13Vin, $z=\mathbf{0 . 8 3 9 8}$

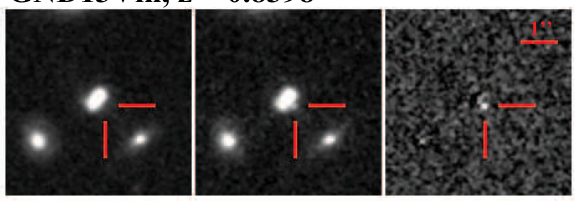

GSD12Roc, $z=0.43 \pm 0.22$

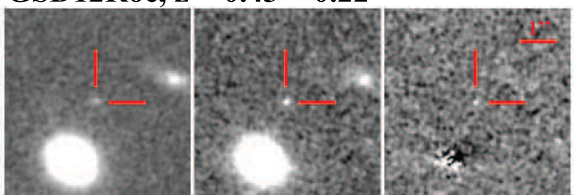

GND13She, $z=0.473$

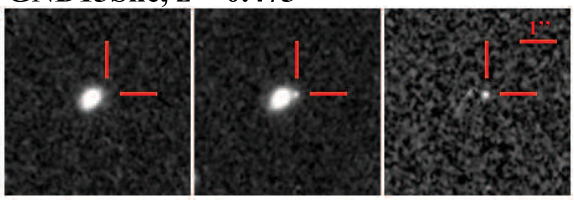

GND13Bid, $z=0.585$

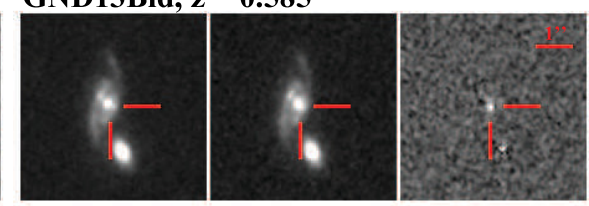

GSD11Wor, $z=0.654$

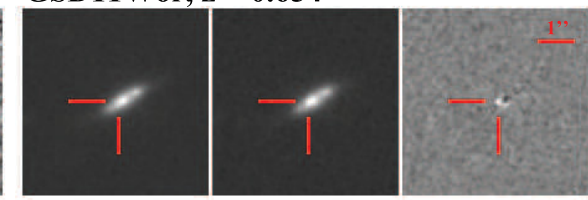

COS12Tru, $z=0.234$

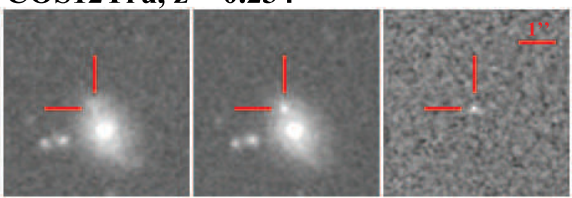

GSD11Har, $z=0.681$

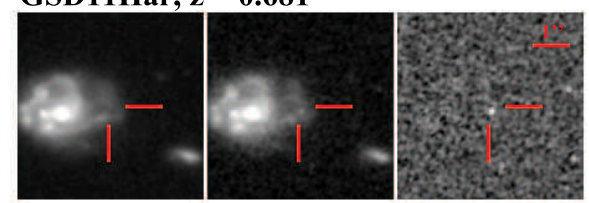

GSD11Ada, $z=0.735$

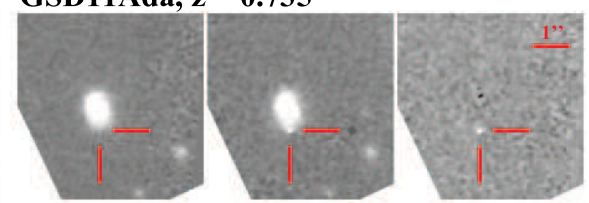

GND12Bur, $\mathrm{z}=0.82 \pm 0.13$

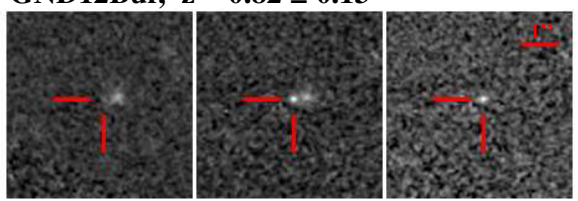

GNW12Ger, $z=0.850$

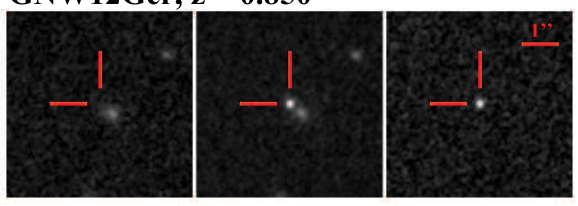

Figure 13. Detection images for $27 \mathrm{SN}$ from the CANDELS fields with redshifts $z \leqslant 0.85$. Each image triplet shows $H$ band (F160W) images with the template image on the left, the discovery epoch image in the middle and the difference image on the right. All images have a width of about 6 arcsec, with north up and east to the left. The position of the SN is marked by (red) crosshairs in every frame.

(A color version of this figure is available in the online journal.) 


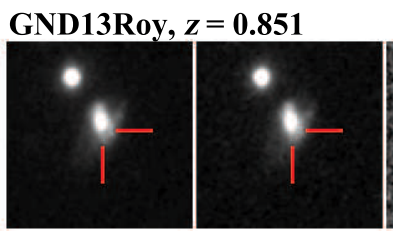

COS12Mik, $z=0.927$

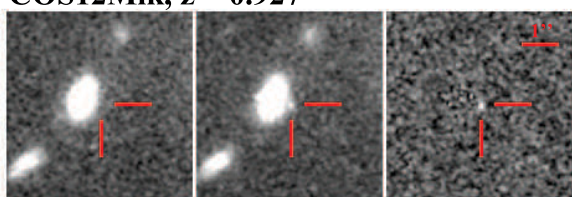

GSD11Mad, $z=0.988$

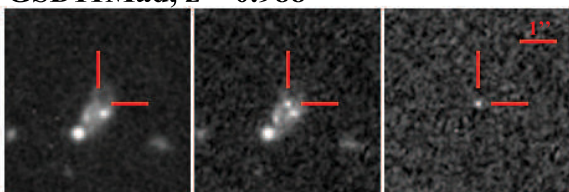

GND13Jay, $z=1.025$

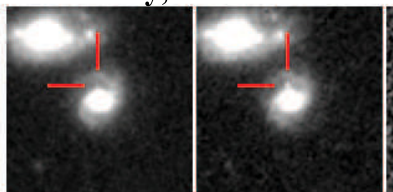

GSD12Agn, $z=1.095$

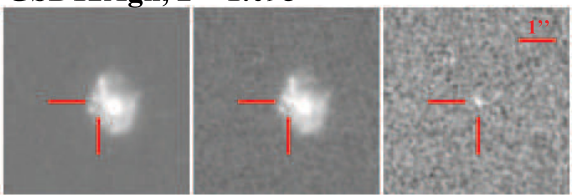

EGS13Wai, $z=1.1662$

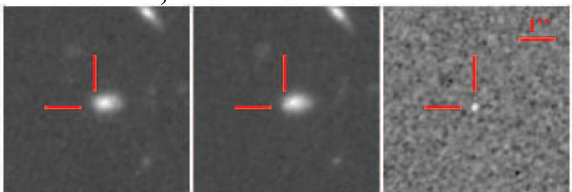

GND12Whe, $z=1.335$

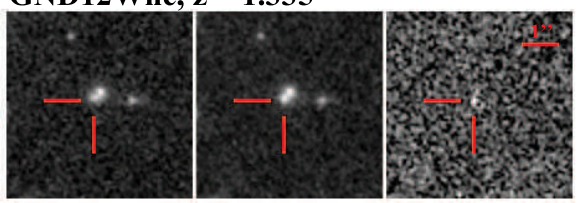

EGS11Nix, $z=1.45 \pm 0.08$

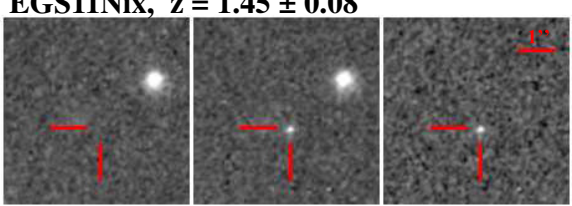

GSD11Van, $\mathrm{z}=0.89 \pm 0.10$

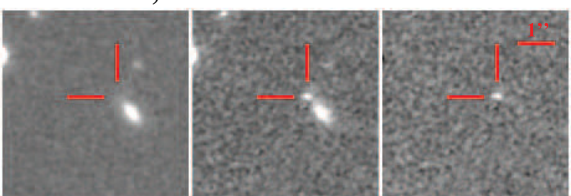

GND12Cal, $z=0.941$

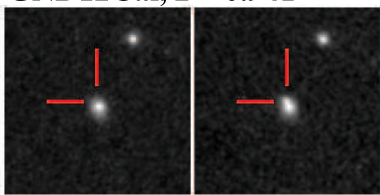

GNW12Gor, $z=1.0164$

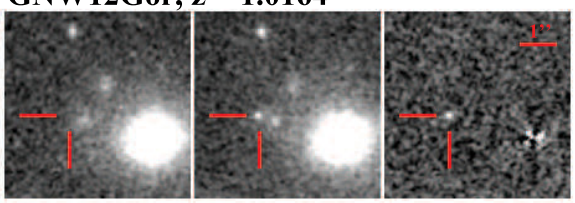

GND13Gar, $z=1.07 \pm 0.02$

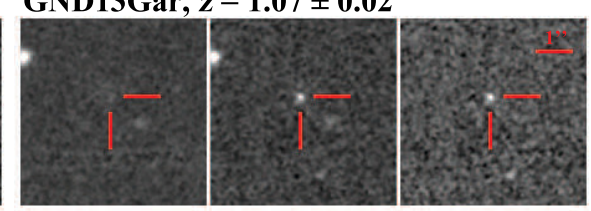

EGS13Tan, $z=1.14 \pm 0.44$

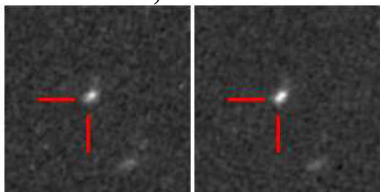

GND13Cam, $z=1.222$

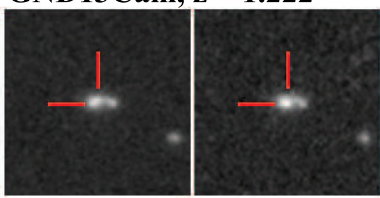

EGS13Cha, $z=1.40 \pm 0.01$

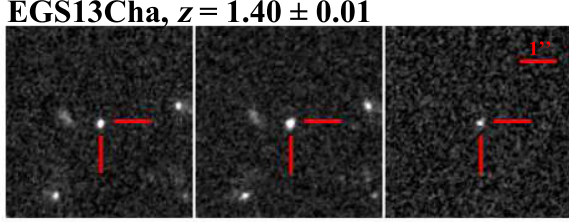

GND12Kin, $z=1.46 \pm 0.07$
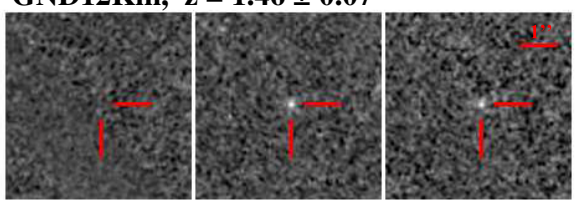

EGS11Per, $z=0.9147$

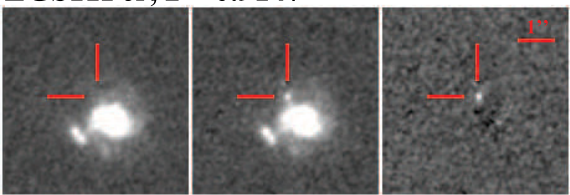

GND13Wol, $z=0.9431$

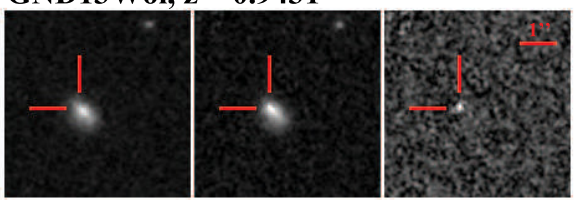

GND13Reh, $z=1.0199$

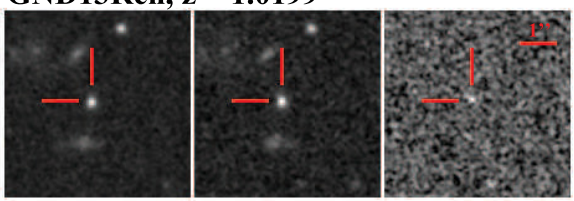

$\operatorname{COS12Her,~} z=1.08 \pm 0.08$

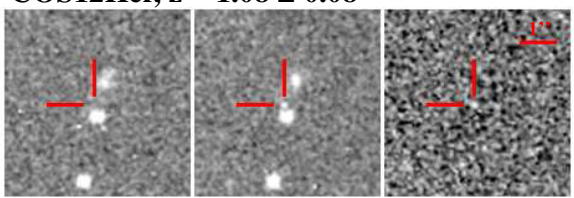

GND12Tom, $z=1.140$

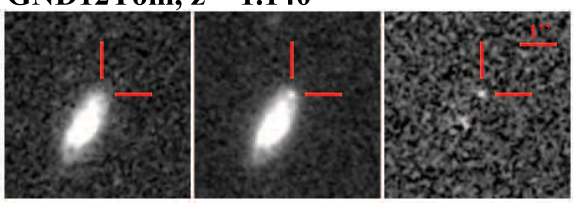

GSD12Was, $z=1.33 \pm 0.02$

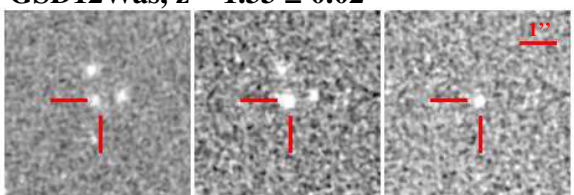

EGS11Oba, $z=1.409$

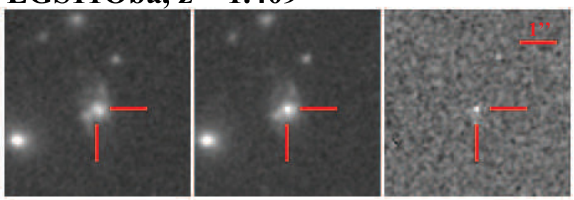

GND13Fin, $z=1.4878$

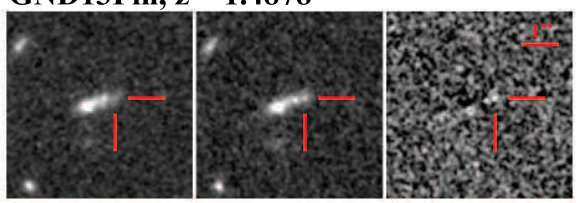

Figure 14. Detection images for $24 \mathrm{SN}$ from the CANDELS fields with redshifts $0.85<z<1.5$. Each image triplet shows $H$ band (F160W) images with the template image on the left, the discovery epoch image in the middle and the difference image on the right. All images have a width of about 6 arcsec, with north up and east to the left. The position of the SN is marked by (red) crosshairs in every frame.

(A color version of this figure is available in the online journal.)

prior $P$ (Ia, host), replacing $P(\mathrm{Ia}, z)$ in Equation (2). Note that this assumption is assuredly incorrect: at redshift $z \sim 2$ the fraction of SNe Ia appearing in red early-type galaxies must be much lower than it is locally, simply because there are far fewer of those passive old galaxies at high redshift. However, for the purpose of this systematic test, we will make the brazen assumption that the relationships between
SN types and their host galaxy properties do not evolve with redshift.

To define our baseline rate measurement, we have relied on the redshift-dependent mid-rate $P(\mathrm{Ia}, z)$ prior (the green solid curve in Figure 6). Because the galsnid-based prior $P$ (Ia,host) prior is redshift independent, we can use it to check for strong redshift biases in the rate-based $P(\mathrm{Ia}, z)$ prior. In principle, these 

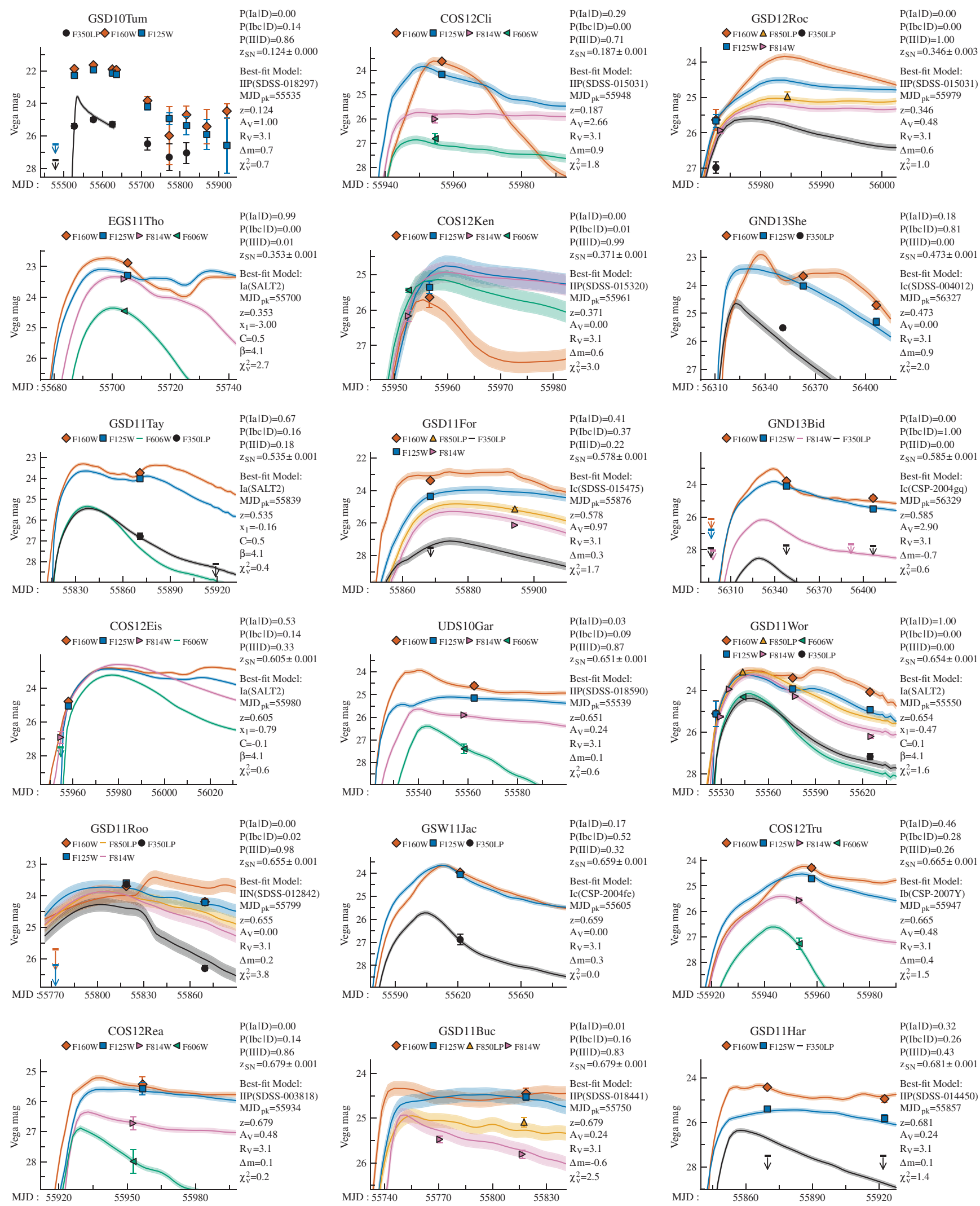

Figure 15. STARDUST light curve matches for the first $18 \mathrm{SN}$ from the CANDELS fields in redshift order, with redshifts $z<0.7$, as in Figure 5 .

(A color version of this figure is available in the online journal.) 

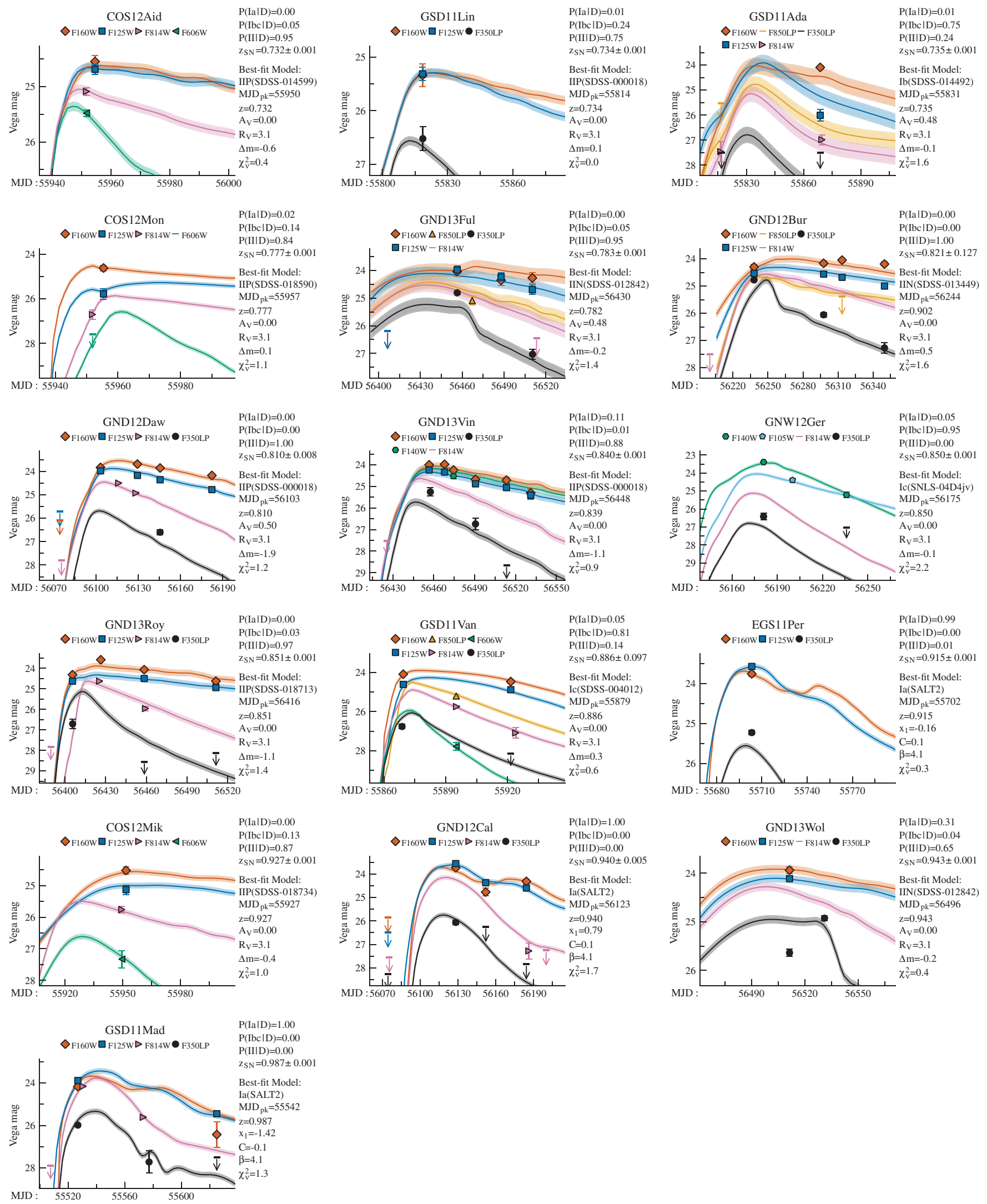

Figure 16. STARDUST light curve matches for the $16 \mathrm{SN}$ from the CANDELS fields with redshifts $0.7<z<1.0$, as in Figure 5 .

(A color version of this figure is available in the online journal.) 

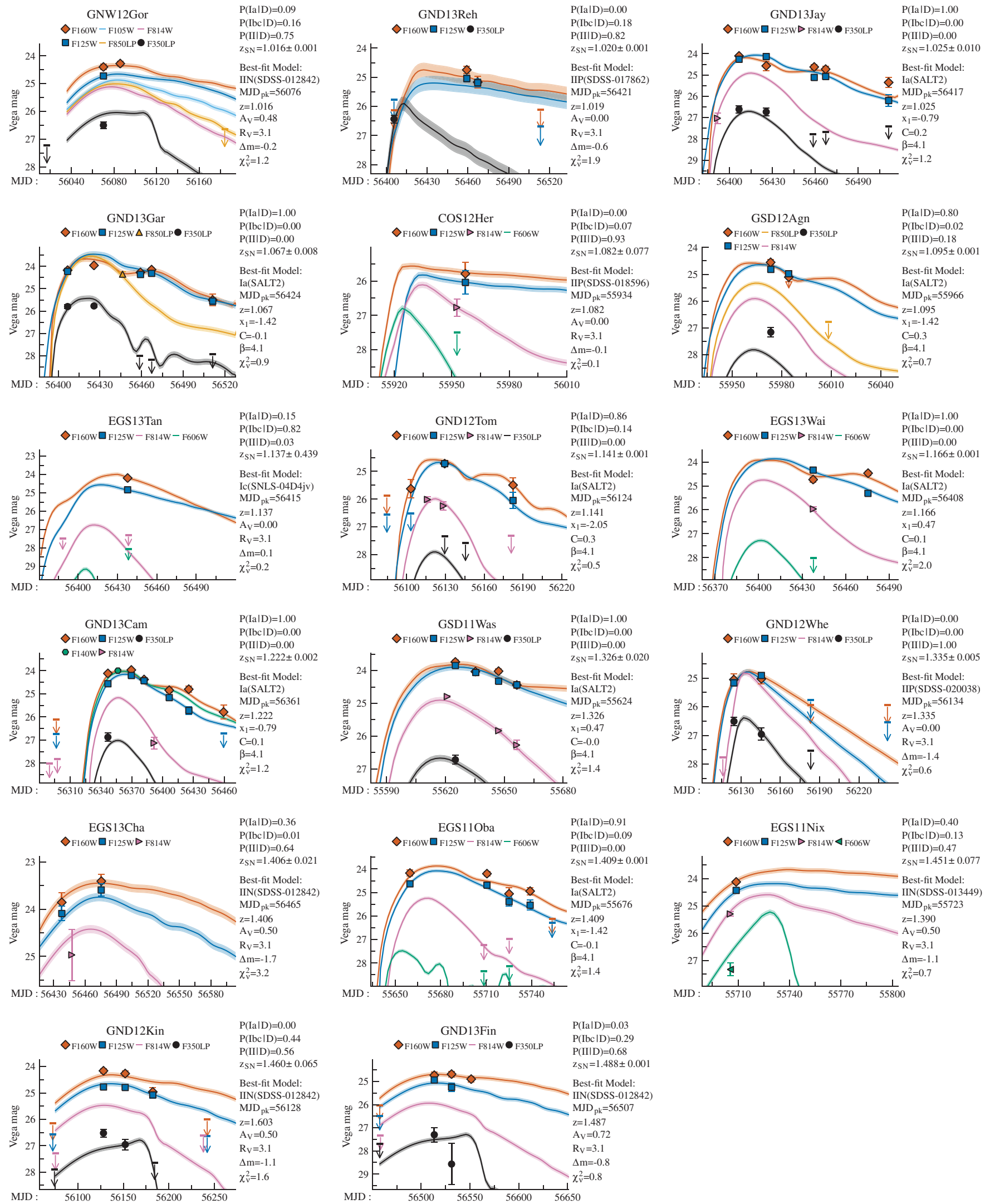

Figure 17. STARDUST light curve matches for the $17 \mathrm{SN}$ from the CANDELS fields with redshifts $1.0<z<1.5$, as in Figure 5 .

(A color version of this figure is available in the online journal.) 
Table 10

Host Galaxies of 51 Supernovae with $z<1.5$

\begin{tabular}{|c|c|c|c|c|c|c|c|c|c|}
\hline $\mathrm{SN}$ & $\begin{array}{c}\text { R.A. } \\
(\mathrm{J} 2000)\end{array}$ & $\begin{array}{c}\text { Decl. } \\
(\mathrm{J} 2000)\end{array}$ & $\begin{array}{c}d \\
\left({ }^{\prime \prime}\right)\end{array}$ & $\begin{array}{c}d \\
(\mathrm{kpc})^{\mathrm{a}}\end{array}$ & Morph. $^{b}$ & $\mathrm{SED}^{\mathrm{c}}$ & Zhost & $( \pm)$ & $z$ Reference $^{\mathrm{d}}$ \\
\hline GSD10Tum & $03: 32: 17.871$ & $-27: 50: 59.48$ & 2.96 & 57.9 & $\mathrm{~d}$ & A & 0.124 & 0.001 & Le Fèvre et al. (2004) \\
\hline COS12Cli & 10:00:16.060 & $+02: 12: 37.38$ & 1.89 & 37.8 & $\mathrm{~d}$ & SB & 0.187 & 0.001 & Trump et al. (2009) \\
\hline GSD12Roc & $03: 32: 06.368$ & $-27: 47: 26.63$ & 0.00 & 0.0 & $\mathrm{i}$ & SB & 0.346 & 0.003 & Keck+DEIMOS (B. Mobasher, S. Jha) \\
\hline EGS11Tho & $14: 19: 31.685$ & $+52: 51: 56.05$ & 0.82 & 16.8 & di & A & 0.354 & 0.001 & Keck+LRIS (A. Filippenko), Gemini+GMOS (S. Jha) \\
\hline COS12Ken & 10:00:35.978 & $+02: 15: 25.81$ & 0.87 & 17.6 & di & SB & 0.373 & 0.001 & Lilly et al. (2009) \\
\hline GND13She & $12: 36: 09.907$ & $+62: 14: 05.79$ & 0.30 & 6.1 & $\mathrm{sd}$ & A & 0.473 & 0.001 & Wirth et al. (2004) \\
\hline GSD11Tay & $03: 32: 54.502$ & $-27: 47: 03.67$ & 0.56 & 11.5 & $\mathrm{~d}$ & A & 0.535 & 0.001 & Le Fèvre et al. (2004) \\
\hline GSD11For & $03: 32: 14.320$ & $-27: 47: 13.15$ & 0.30 & 6.3 & d & A & 0.578 & 0.001 & Mignoli et al. (2005) \\
\hline GND13Bid & $12: 36: 41.417$ & $+62: 11: 42.53$ & 0.72 & 14.7 & $\mathrm{i}$ & A & 0.585 & 0.001 & Cohen et al. (2000) \\
\hline COS12Eis & 10:00:47.275 & $+02: 11: 50.04$ & 0.78 & 15.9 & sd & SB & 0.605 & 0.001 & Keck+DEIMOS (B. Mobasher) \\
\hline UDS11Gar & $02: 17: 39.631$ & $-05: 11: 37.00$ & 0.14 & 3.0 & $\mathrm{sd}$ & SB & 0.651 & 0.001 & Keck+DEIMOS (B. Mobasher) \\
\hline GSD11Wor & $03: 32: 10.730$ & $-27: 48: 07.14$ & 0.12 & 2.6 & $\mathrm{~d}$ & A & 0.654 & 0.001 & Mignoli et al. (2005) \\
\hline GSD11Roo & $03: 32: 31.581$ & $-27: 46: 12.71$ & 0.20 & 4.1 & $\mathrm{~s}$ & SB & 0.655 & 0.001 & VLT+FORS2 (B. Leibundgut) \\
\hline GSW11Jac & $03: 32: 32.310$ & $-27: 54: 20.46$ & 0.16 & 3.3 & $\mathrm{~d}$ & A & 0.659 & 0.001 & VLT+Xshooter (J. Hjorth) \\
\hline COS12Tru & $10: 00: 38.293$ & $+02: 11: 35.60$ & 0.92 & 18.7 & $\mathrm{~d}$ & A & 0.665 & 0.001 & Keck+DEIMOS (B. Mobasher) \\
\hline COS12Rea & 10:00:32.021 & $+02: 14: 15.43$ & 1.72 & 34.4 & $\mathrm{~d}$ & SB & 0.679 & 0.001 & Lilly et al. (2009) \\
\hline GSD11Buc & $03: 32: 28.714$ & $-27: 52: 32.00$ & 1.14 & 23.1 & $\mathrm{~s}$ & A & 0.679 & 0.001 & Le Fèvre et al. (2004) \\
\hline GSD11Har & $03: 32: 30.570$ & $-27: 45: 18.35$ & 1.75 & 35.0 & $\mathrm{~d}$ & SB & 0.681 & 0.001 & Le Fèvre et al. (2004), Mignoli et al. (2005) \\
\hline COS12Aid & 10:00:15.212 & $+02: 17: 30.84$ & 1.41 & 28.5 & $\mathrm{sd}$ & SB & 0.731 & 0.001 & Keck+DEIMOS (B. Mobasher) \\
\hline GSD11Lin & 03:32:29.799 & $-27: 49: 19.26$ & 0.48 & 9.9 & $\mathrm{sd}$ & SB & 0.734 & 0.001 & Keck+DEIMOS (A. Filippenko, S. Jha) \\
\hline GSD11Ada & $03: 32: 19.785$ & $-27: 54: 09.15$ & 0.93 & 18.9 & $\mathrm{sd}$ & SB & 0.735 & 0.001 & Balestra et al. (2010), Popesso et al. (2009) \\
\hline COS12Mon & 10:00:26.746 & $+02: 15: 14.02$ & 0.31 & 6.4 & $\mathrm{~s}$ & SB & 0.777 & 0.001 & Lilly et al. (2009) \\
\hline GND13Ful & $12: 36: 19.227$ & $+62: 15: 12.76$ & 0.26 & 5.3 & sd & SB & 0.783 & 0.001 & Wirth et al. (2004) \\
\hline GND12Bur & $12: 36: 32.464$ & $+62: 15: 32.75$ & 0.52 & 10.7 & $\mathrm{i}$ & SB & 0.69 & 0.4 & phot-z (T. Dahlen) \\
\hline GND12Daw & $\cdots$ & $\cdots$ & $\cdots$ & $\cdots$ & $\cdots$ & $\cdots$ & $\cdots$ & $\cdots$ & $\cdots$ \\
\hline GND13Vin & $12: 37: 06.308$ & $+62: 15: 18.08$ & 0.43 & 8.9 & $\mathrm{i}$ & A & 0.840 & 0.001 & Cohen et al. (2000) \\
\hline GNW12Ger & $12: 37: 16.965$ & $+62: 20: 38.34$ & 0.52 & 10.7 & $\mathrm{~s}$ & A & 0.850 & 0.005 & Keck+DEIMOS (A. Filippenko) \\
\hline GND13Roy & $12: 36: 46.232$ & $+62: 15: 27.46$ & 0.34 & 6.9 & $\mathrm{i}$ & SB & 0.851 & 0.001 & Cooper et al. (2011) \\
\hline GSD11Van & $03: 32: 19.004$ & $-27: 47: 18.33$ & 0.61 & 12.6 & $\mathrm{~d}$ & $\mathrm{P}$ & 0.74 & 0.15 & phot-z (T. Dahlen) \\
\hline EGS11Per & $14: 18: 28.248$ & $+52: 42: 45.07$ & 1.03 & 20.9 & $\mathrm{~d}$ & A & 0.915 & 0.001 & Barro et al. (2011) \\
\hline COS12Mik & $10: 00: 31.733$ & $+02: 26: 13.92$ & 0.72 & 14.6 & $\mathrm{sd}$ & SB & 0.927 & 0.001 & Keck+DEIMOS (B. Mobasher) \\
\hline GND12Cal & $12: 37: 10.476$ & $+62: 15: 47.40$ & 0.28 & 5.8 & $\mathrm{~s}$ & SB & 0.941 & 0.005 & HST+WFC3 (B. Weiner) \\
\hline GND13Wol & $12: 36: 58.933$ & $+62: 18: 10.04$ & 0.14 & 3.0 & $\mathrm{sd}$ & SB & 0.9431 & 0.0002 & Barger et al. (2008) \\
\hline GSD11Mad & $03: 32: 18.767$ & $-27: 52: 42.45$ & 0.44 & 9.1 & $\mathrm{~d}$ & SB & 0.988 & 0.001 & Keck+LRIS (A. Filippenko) \\
\hline GNW12Gor & $12: 36: 20.619$ & $+62: 08: 44.91$ & 0.62 & 12.7 & $\mathrm{u}$ & A & 1.0164 & 0.0005 & Wirth et al. (2004), Treu et al. (2005) \\
\hline GND13Reh & $12: 36: 54.445$ & $+62: 11: 52.43$ & 0.06 & 1.3 & $\mathrm{~s}$ & SB & 1.019 & 0.001 & Barger et al. (2008) \\
\hline GND13Jay & $12: 36: 41.370$ & $+62: 11: 29.57$ & 0.46 & 9.4 & $\mathrm{~d}$ & A & 1.03 & 0.01 & HST+WFC3 (B. Weiner) \\
\hline GND13Gar & $12: 36: 40.813$ & $+62: 11: 14.34$ & 0.19 & 3.9 & $\mathrm{u}$ & SB & 1.07 & 0.02 & phot- $z$ (T. Dahlen) \\
\hline COS12Her & $10: 00: 47.438$ & $+02: 15: 17.35$ & 0.41 & 8.4 & $\mathrm{~s}$ & SB & 1.1 & 0.4 & Keck+DEIMOS (B. Mobasher) \\
\hline GSD12Agn & $03: 32: 25.861$ & $-27: 50: 19.81$ & 0.58 & 11.8 & $\mathrm{~d}$ & SB & 1.095 & 0.001 & Vanzella et al. (2008) \\
\hline EGS13Tan & $14: 20: 02.010$ & $+53: 00: 17.04$ & 1.05 & 21.3 & $\mathrm{sd}$ & A & 1.39 & 0.8 & phot-z (T. Dahlen) \\
\hline GND12Tom & $12: 36: 42.636$ & $+62: 18: 20.74$ & 1.36 & 27.4 & $\mathrm{~d}$ & A & 1.140 & 0.001 & Barger et al. (2008) \\
\hline EGS13Wai & $14: 20: 28.495$ & $+53: 04: 58.72$ & 0.37 & 7.6 & di & SB & 1.1662 & 0.0004 & Barro et al. (2011) \\
\hline GND13Cam & $12: 37: 07.357$ & $+62: 10: 26.94$ & 0.05 & 0.9 & $\mathrm{sd}$ & SB & 1.222 & 0.002 & HST+WFC3 (B. Weiner) \\
\hline GSD11Was & $03: 32: 20.856$ & $-27: 49: 41.48$ & 0.00 & 0.0 & $\mathrm{~d}$ & SB & 1.30 & 0.05 & HST+WFC3 (A. Riess) \\
\hline GND12Whe & $12: 36: 24.206$ & $+62: 17: 39.92$ & 0.24 & 5.0 & $\mathrm{sd}$ & SB & 1.335 & 0.001 & Keck+DEIMOS (C. Papovich) \\
\hline EGS13Cha & $14: 20: 24.200$ & $+53: 03: 37.50$ & 0.00 & 0.0 & $\mathrm{sd}$ & A & 1.40 & 0.05 & Keck+MOSFIRE (M. Cooper) \\
\hline EGS11Oba & $14: 20: 32.666$ & $+53: 02: 48.10$ & 0.08 & 1.8 & di & A & 1.409 & 0.001 & Keck+LRIS (A. Filippenko) \\
\hline EGS11Nix & $14: 20: 48.607$ & $+53: 00: 26.56$ & 0.10 & 2.0 & $\mathrm{u}$ & A & 1.73 & 0.51 & phot- $z$ (T. Dahlen) \\
\hline GND12Kin & $12: 37: 13.013$ & $+62: 16: 30.86$ & 0.06 & 1.3 & $\mathrm{u}$ & SB & 1.9 & 0.4 & phot-z (T. Dahlen) \\
\hline GND13Fin & $12: 37: 18.322$ & $+62: 17: 09.34$ & 1.08 & 22.0 & $\mathrm{i}$ & SB & 1.4878 & 0.0005 & Wirth et al. (2004), Reddy et al. (2006) \\
\hline
\end{tabular}

Notes.

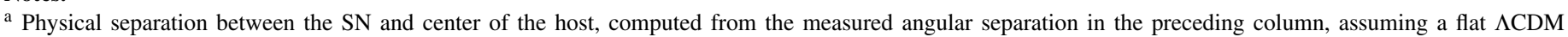
cosmology with $H_{0}=70, \Omega_{\mathrm{m}}=0.3$.

b Visual classifications for host galaxy morphology: $\mathrm{s}=$ spheroid, $\mathrm{d}=$ disk, $\mathrm{i}=$ irregular.

c Template-matching classification of host galaxy SED: $\mathrm{P}=$ Passive, $\mathrm{A}=$ Active, $\mathrm{SB}=$ Starburst type.

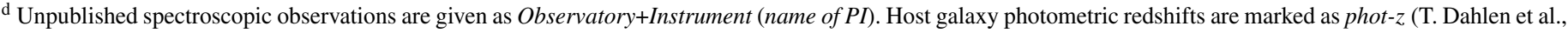
in preparation).

two priors could be combined into a redshift-dependent, hostbased prior-but that is beyond the scope of this paper.

In Column 5 of Tables 3 and 9 we have reported the STARDUST probabilities derived using this host galaxy based prior. As one should expect, SNe for which we have abundant spectroscopic and photometric information are barely affected. Thus, classification probabilities that were close to 0 or 1 using the redshift-dependent prior typically do not shift. For objects 
with intermediate probabilities, the Ia classification probability is almost uniformly increased, but the change is mostly within the range allowed by the classification uncertainties. The total change in the count of observed $\mathrm{SNe}$ Ia and the resulting change in the volumetric SN rate are presented in Table 8. Again, the shift is uniformly positive, but relatively small when compared to systematic uncertainties. The final column of this table reports the change in the SN Ia rate measurement as a fraction of the systematic uncertainty estimate, and we see that it is less than unity in every redshift bin. While not definitive, this result suggests that our baseline rates are not heavily biased by the redshift-dependent class prior, and any existing bias has been sufficiently accounted for in our systematic uncertainty estimates.

\section{APPENDIX B}

\section{SUPPLEMENTARY DATA FOR SNe AT $z<1.5$}

Discovery images for the $51 \mathrm{SNe}$ with redshift $z<1.5$ are shown in Figures 13 and 14. The names, positions, classification probabilities, and redshifts of those 51 low- $z$ SNe are given in Table 9 (as in Table 3). Host galaxy information is provided in Table 10 (as in Table 4). Light curves and best-fitting template matches from STARDUST are shown in Figures 15-17.

\section{REFERENCES}

Alard, C., \& Lupton, R. H. 1998, ApJ, 503, 325

Astier, P., Guy, J., Regnault, N., et al. 2006, A\&A, 447, 31

Astropy Collaboration, Robitaille, T. P., Tollerud, E. J., et al. 2013, A\&A, 558, A33

Balestra, I., Mainieri, V., Popesso, P., et al. 2010, A\&A, 512, A12

Barbary, K., Aldering, G., Amanullah, R., et al. 2012, ApJ, 745, 31

Barger, A. J., Cowie, L. L., \& Wang, W.-H. 2008, ApJ, 689, 687

Barro, G., Pérez-González, P. G., Gallego, J., et al. 2011, ApJS, 193, 13

Behroozi, P. S., Wechsler, R. H., \& Conroy, C. 2013, ApJ, 770, 57

Belczynski, K., Bulik, T., \& Ruiter, A. J. 2005, ApJ, 629, 915

Bildsten, L., Shen, K. J., Weinberg, N. N., \& Nelemans, G. 2007, ApJL, 662, L95

Blanc, G., Afonso, C., Alard, C., et al. 2004, A\&A, 423, 881

Blondin, S., \& Tonry, J. L. 2007, ApJ, 666, 1024

Botticella, M. T., Riello, M., Cappellaro, E., et al. 2008, A\&A, 479, 49

Bours, M. C. P., Toonen, S., \& Nelemans, G. 2013, A\&A, 552, A24

Cappellaro, E., Evans, R., \& Turatto, M. 1999, A\&A, 351, 459

Chabrier, G. 2003, PASP, 115, 763

Cirasuolo, M., McLure, R. J., Dunlop, J. S., et al. 2007, MNRAS, 380, 585

Claeys, J. S. W., Pols, O. R., Izzard, R. G., Vink, J., \& Verbunt, F. W. M. 2014, A\&A, 563, A83

Cohen, J. G., Hogg, D. W., Blandford, R., et al. 2000, ApJ, 538, 29

Cooper, M. C., Aird, J. A., Coil, A. L., et al. 2011, ApJS, 193, 14

Dahlen, T., Mobasher, B., Dickinson, M., et al. 2010, ApJ, 724, 425

Dahlen, T., Mobasher, B., Faber, S. M., et al. 2013, ApJ, 775, 93

Dahlen, T., Strolger, L.-G., \& Riess, A. G. 2008, ApJ, 681, 462

Dahlen, T., Strolger, L.-G., Riess, A. G., et al. 2004, ApJ, 613, 189

Dahlen, T., Strolger, L.-G., Riess, A. G., et al. 2012, ApJ, 757, 70

D’Andrea, C. B., Sako, M., Dilday, B., et al. 2010, ApJ, 708, 661

Davis, M., Guhathakurta, P., Konidaris, N. P., et al. 2007, ApJL, 660, L1

Dilday, B., Smith, M., Bassett, B., et al. 2010, ApJ, 713, 1026

Drout, M. R., Soderberg, A. M., Gal-Yam, A., et al. 2011, ApJ, 741, 97

Foley, R. J., Challis, P. J., Chornock, R., et al. 2013, ApJ, 767, 57

Foley, R. J., \& Mandel, K. 2013, ApJ, 778, 167

Frederiksen, T. F., Graur, O., Hjorth, J., Maoz, D., \& Poznanski, D. 2014, A\&A, 563,140

Frederiksen, T. F., Hjorth, J., Maund, J. R., et al. 2012, ApJ, 760, 125

Frieman, J. A., Turner, M. S., \& Huterer, D. 2008, ARA\&A, 46, 385

Fruchter, A. S., \& Hook, R. N. 2002, PASP, 114, 144

Gal-Yam, A. 2012, Sci, 337, 927

Giavalisco, M., Ferguson, H. C., Koekemoer, A. M., et al. 2004, ApJL, 600, L93

Graur, O., \& Maoz, D. 2013, MNRAS, 430, 1746

Graur, O., Poznanski, D., Maoz, D., et al. 2011, MNRAS, 417, 916
Graur, O., Rodney, S. A., Maoz, D., et al. 2014, ApJ, 783, 28

Greggio, L. 2010, MNRAS, 406, 22

Grogin, N. A., Kocevski, D. D., Faber, S. M., et al. 2011, ApJS, 197, 35

Guy, J., Sullivan, M., Conley, A., et al. 2010, A\&A, 523, A7

Hachisu, I., Kato, M., \& Nomoto, K. 1996, ApJL, 470, L97

Hachisu, I., Kato, M., \& Nomoto, K. 1999, ApJ, 522, 487

Hamuy, M., Folatelli, G., Morrell, N. I., et al. 2006, PASP, 118, 2

Hardin, D., Afonso, C., Alard, C., et al. 2000, A\&A, 362, 419

Hillebrandt, W., \& Niemeyer, J. 2000, ARA\&A, 38, 191

Horesh, A., Poznanski, D., Ofek, E. O., \& Maoz, D. 2008, MNRAS, 389, 1871

Iben, I., Jr., \& Tutukov, A. V. 1984, ApJS, 54, 335

Jones, D. O., Rodney, S. A., Riess, A. G., et al. 2013, ApJ, 768, 166

Kartaltepe, J. S., Dickinson, M., Alexander, D. M., et al. 2012, ApJ, 757, 23

Kashi, A., \& Soker, N. 2011, MNRAS, 417, 1466

Kelly, P. L., Kirshner, R. P., \& Pahre, M. 2008, ApJ, 687, 1201

Kessler, R., Becker, A. C., Cinabro, D., et al. 2009a, ApJS, 185, 32

Kessler, R., Bernstein, J. P., Cinabro, D., et al. 2009b, PASP, 121, 1028

Kiewe, M., Gal-Yam, A., Arcavi, I., et al. 2012, ApJ, 744, 10

Kobayashi, C., \& Nomoto, K. 2009, ApJ, 707, 1466

Kobayashi, C., Tsujimoto, T., Nomoto, K., Hachisu, I., \& Kato, M. 1998, ApJL, $503, \mathrm{~L} 155$

Koekemoer, A. M., Aussel, H., Calzetti, D., et al. 2007, ApJS, 172, 196

Koekemoer, A. M., Faber, S. M., Ferguson, H. C., et al. 2011, ApJS, 197, 36

Koekemoer, A. M., Fruchter, A. S., Hook, R. N., \& Hack, W. 2002, in The 2002

HST Calibration Workshop, ed. S. Arribas, A. Koekemoer, \& B. Whitmore (Baltimore, MD: Space Telescope Institute), 337

Krist, J. E., Hook, R. N., \& Stoehr, F. 2011, Proc. SPIE, 8127, 16

Kuznetsova, N., Barbary, K., Connolly, B., et al. 2008, ApJ, 673, 981

Lawrence, A., Warren, S. J., Almaini, O., et al. 2007, MNRAS, 379, 1599

Le Fèvre, O., Vettolani, G., Paltani, S., et al. 2004, A\&A, 428, 1043

Leaman, J., Li, W., Chornock, R., \& Filippenko, A. V. 2011, MNRAS, 412, 1419

Li, W., Leaman, J., Chornock, R., et al. 2011, MNRAS, 412, 1441

Lilly, S. J., Le Brun, V., Maier, C., et al. 2009, ApJS, 184, 218

Livio, M. 2001, in SNe and GRBs, ed. M. Livio, N. Panagia, \& K. Sahu (Cambridge: Cambridge Univ. Press), 334

Madau, P., della Valle, M., \& Panagia, N. 1998, MNRAS, 297, L17

Mannucci, F., Della Valle, M., \& Panagia, N. 2006, MNRAS, 370, 773

Mannucci, F., Della Valle, M., \& Panagia, N. 2007, MNRAS, 377, 1229

Mannucci, F., Della Valle, M., Panagia, N., et al. 2005, A\&A, 433, 807

Maoz, D., \& Badenes, C. 2010, MNRAS, 407, 1314

Maoz, D., \& Mannucci, F. 2012, PASA, 29, 447

Mattila, S., Dahlen, T., Efstathiou, A., et al. 2012, ApJ, 756, 111

Melinder, J., Dahlen, T., Mencía Trinchant, L., et al. 2012, A\&A, 545, A96

Mennekens, N., Vanbeveren, D., De Greve, J. P., \& De Donder, E. 2010, A\&A, 515, A89

Mennekens, N., Vanbeveren, D., De Greve, J.-P., \& De Donder, E. 2013, in IAU Symp. 281, Binary Paths to Type Ia Supernovae Explosions, ed. R. Di Stefano, M. Orio, \& M. Moe (Cambridge: Cambridge Univ. Press), 232

Mignoli, M., Cimatti, A., Zamorani, G., et al. 2005, A\&A, 437, 883

Morrell, N. I. 2012, in IAU Symp. 279, Death of Massive Stars: Supernovae and Gamma-Ray Bursts, ed. P. Roming, N. Kawai, \& E. Pian (Cambridge: Cambridge Univ. Press), 361

Murphy, E. J., Chary, R.-R., Alexander, D. M., et al. 2009, ApJ, 698, 1380

Neill, J. D., Sullivan, M., Balam, D., et al. 2006, AJ, 132, 1126

Pain, R., Fabbro, S., Sullivan, M., et al. 2002, ApJ, 577, 120

Paterno, M. 2004, FERMILAB-TM-2286-CD

Perrett, K., Sullivan, M., Conley, A., et al. 2012, AJ, 144, 59

Popesso, P., Dickinson, M., Nonino, M., et al. 2009, A\&A, 494, 443

Postman, M., Coe, D., Benítez, N., et al. 2012, ApJS, 199, 25

Poznanski, D., Maoz, D., Yasuda, N., et al. 2007, MNRAS, 382, 1169

Quimby, R. M., Kulkarni, S. R., Kasliwal, M. M., et al. 2011, Natur, 474, 487

Raskin, C., Scannapieco, E., Rhoads, J., \& Della Valle, M. 2009, ApJ, 707, 74

Reddy, N. A., Steidel, C. C., Erb, D. K., Shapley, A. E., \& Pettini, M. 2006, ApJ, 653,1004

Riess, A. G., Strolger, L.-G., Casertano, S., et al. 2007, ApJ, 659, 98

Rodney, S. A., Riess, A. G., Dahlen, T., et al. 2012, ApJ, 746, 5

Rodney, S. A., \& Tonry, J. L. 2010, ApJ, 723, 47

Ruiter, A. J., Belczynski, K., \& Fryer, C. 2009, ApJ, 699, 2026

Ruiter, A. J., Sim, S. A., Pakmor, R., et al. 2013, MNRAS, 429, 1425

Sako, M., Bassett, B., Becker, A., et al. 2008, AJ, 135, 348

Scannapieco, E., \& Bildsten, L. 2005, ApJL, 629, L85

Scolnic, D. M., Riess, A. G., Foley, R. J., et al. 2014, ApJ, 780, 37

Scoville, N., Aussel, H., Brusa, M., et al. 2007, ApJS, 172, 1

Sharon, K., Gal-Yam, A., Maoz, D., Filippenko, A. V., \& Guhathakurta, P. 2007, ApJ, 660, 1165 
Smartt, S. J., Eldridge, J. J., Crockett, R. M., \& Maund, J. R. 2009, MNRAS, 395, 1409

Stritzinger, M., Mazzali, P., Phillips, M. M., et al. 2009, ApJ, 696, 713

Strolger, L., Dahlen, T., \& Riess, A. G. 2010, ApJ, 713, 32

Strolger, L.-G., Riess, A. G., Dahlen, T., et al. 2004, ApJ, 613, 200

Thompson, T. A. 2011, ApJ, 741, 82

Tonry, J. L., Schmidt, B. P., Barris, B., et al. 2003, ApJ, 594, 1

Treu, T., Ellis, R. S., Liao, T. X., \& van Dokkum, P. G. 2005, ApJL, 622, L5
Trump, J. R., Impey, C. D., Elvis, M., et al. 2009, ApJ, 696, 1195

Vanzella, E., Cristiani, S., Dickinson, M., et al. 2008, A\&A, 478, 83

Wang, B., Chen, X., Meng, X., \& Han, Z. 2009, ApJ, 701, 1540

Webbink, R. F. 1984, ApJ, 277, 355

Whelan, J., \& Iben, I. J. 1973, ApJ, 186, 1007

Wiersma, R. P. C., Schaye, J., \& Theuns, T. 2011, MNRAS, 415, 353

Wirth, G. D., Willmer, C. N. A., Amico, P., et al. 2004, AJ, 127, 3121 